\title{
Assumptions of the process-dissociation procedure are violated in implicit sequence learning
}

\author{
Marius Barth, Christoph Stahl, \& Hilde Haider \\ University of Cologne
}

(Unpublished manuscript, 2018-03-09).

\begin{abstract}
In implicit sequence learning, a process-dissociation (PD) approach has been proposed to dissociate implicit and explicit learning processes. Applied to the popular generation task, participants perform two different task versions: inclusion instructions require generating the transitions that form the learned sequence; exclusion instructions require generating transitions other than those of the learned sequence. Whereas accurate performance under inclusion may be based on either implicit or explicit knowledge, avoiding to generate learned transitions requires controllable explicit sequence knowledge. The PD approach yields separate estimates of explicit and implicit knowledge that are derived from the same task; it therefore avoids many problems of previous measurement approaches. However, the PD approach rests on the critical assumption that the implicit and explicit processes are invariant across inclusion and exclusion conditions. We tested whether the invariance assumptions hold for the PD generation task. Across three studies using firstorder as well as second-order regularities, invariance of the controlled process was found to be violated. In particular, despite extensive amounts of practice, explicit knowledge was not exhaustively expressed in the exclusion condition. We discuss the implications of these findings for the use of process-dissociation in assessing implicit knowledge.
\end{abstract}

Keywords: sequence learning, process-dissociation procedure, invariance assumption

Word count: 14,936

Riding a bicycle is an easy task, but most of us will be hard-pressed to describe in detail the coordinated movements necessary for pedaling, keeping direction, and maintaining balance. Capturing this intuition, theories of human learning commonly distinguish two types of knowledge: Explicit learning that is accompanied by awareness of its contents, and implicit learning that operates independently of awareness (Shanks \& St. John, 1994).

Such implicit learning has been demonstrated using the Serial Reaction Time Task 
(SRTT, Nissen \& Bullemer, 1987), which has participants respond to stimuli presented at four horizontal screen locations by pressing the key that corresponds to the stimulus location. Unbeknownst to participants, the stimulus locations follow a regular sequence. With practice, participants learn to respond faster on trials with regular stimulus-location transitions than on irregular transitions. Critically, on a subsequent task, participants are often not able to express explicit knowledge about the sequential structure (Cohen, Ivry, \& Keele, 1990; Nissen \& Bullemer, 1987; Willingham, Nissen, \& Bullemer, 1989).

There has been a long-lasting debate whether or not this effect is evidence for implicit learning, a question entwined with methodological considerations of how to properly measure and separate the contributions of supposedly implicit and/or explicit learning systems to this task (for a recent review, see Timmermans \& Cleeremans, 2015). One of the most promising methods has been the process-dissociation (PD) approach as applied to the free generation task (Destrebecqz \& Cleeremans, 2001); yet, its validity rests on a set of previously untested assumptions. The present study assesses two of the crucial assumptions on which this method is based.

\section{Measuring implicit knowledge in the SRTT}

In order to conclude that the learning effect in the SRTT (i.e., an RT advantage for regular transitions) is based on implicit knowledge, dissociations from subsequent assessments of explicit knowledge are typically sought. They depend on the assumptions that the explicit task is as sensitive to explicit sequence knowledge as the SRTT (the absence of an explicit effect may otherwise be due to lower reliability); and that it is also an exhaustive and exclusive measure of explicit knowledge, such that performance on the explicit task reflects all explicit but no implicit knowledge (Reingold \& Merikle, 1990; Shanks \& St. John, 1994). Multiple explicit-knowledge assessment tasks have been proposed, including verbal reports (i.e., recall of the sequence), recognition, prediction, and generation tests. Yet, while dissociations from RT advantages in the SRTT have been demonstrated in some studies, these tests have also been criticized for not meeting the above criteria, or the reported dissociations did not replicate (Shanks \& Perruchet, 2002).

Contrary to the reported dissociations, studies utilizing recognition tests typically found substantial associations of the RT advantage in the SRTT with explicit knowledge (Perruchet \& Amorim, 1992; Perruchet \& Gallego, 1993; Perruchet, Bigand, \& Benoit-Gonin, 1997). It has been argued that these associations were found because the subsequently used recognition task might not be exclusive to explicit but might also be driven by fluency-

Marius Barth, Christoph Stahl, Hilde Haider, Department of Psychology, University of Cologne.

CS and HH designed the research; MB, CS, and HH planned the studies; MB conducted the studies; MB and CS analyzed the data; MB, CS, and HH wrote the paper. We thank Jan Czarnomski, Conni Lebbing, Friederike Neugebauer, and Imge Ürer for help with data collection.

This work was funded by Deutsche Forschungsgemeinschaft grant STA-1269/1-1 to CS and grant HA$5447 / 8-1$ to $\mathrm{HH}$.

Data, code, and materials necessary to reproduce the analyses reported in this article are available at https://github.com/methexp/pdl2.

Correspondence concerning this article should be addressed to Marius Barth, Herbert-Lewin-Str. 2, D-50931 Köln, Germany. E-mail: marius.barth@uni-koeln.de 
based processes. To test this alternative explanation, Buchner and colleagues (Buchner, Erdfelder, Steffens, \& Martensen, 1997; Buchner, Steffens, \& Rothkegel, 1998) used the process-dissociation approach to disentangle (explicit) recollection and (implicit) fluency in the recognition task, finding that recognition is in fact driven by both processes. Still, Shanks and Johnstone (1999) argued that fluency-based recognition judgments cannot be equated with implicit knowledge, leading them to conclude that there was no conclusive evidence for implicit learning in the SRTT literature.

Given the interpretative problems of the recognition task, Destrebecqz and Cleeremans (2001) introduced the process-dissociation approach to the free-generation task, a measure that was considered to be the most sensitive to sequence learning (Perruchet \& Amorim, 1992). Participants were instructed, after finishing the SRTT, to generate a sequence that is either similar (in the inclusion condition) or dissimilar (in the exclusion condition) to that encountered during the SRTT. If participants can generate a similar sequence under the inclusion instruction, they can be said to have acquired knowledge about the sequence; yet, this knowledge may reflect both implicit and explicit knowledge because both may be used to re-generate the learned sequence. However, only explicit knowledge is assumed to be under participants' control: When asked to generate a sequence that is dissimilar to the learned sequence - that is, to exclude their explicit knowledge - participants can avoid generating similar transitions only if their sequence knowledge is explicit. If, instead, their sequence knowledge is implicit, they would still generate a sequence similar to the learned sequence despite being instructed to do the opposite.

To selectively impair explicit knowledge, Destrebecqz and Cleeremans (2001) manipulated the (presence versus absence of a) response-stimulus interval (RSI), speculating that a certain minimal amount of preparation time would be necessary to acquire explicit knowledge during the SRTT. In both an RSI and a no-RSI condition, performance in the free-generation task was above a chance baseline, corroborating previous findings that the generation task is sensitive to sequence knowledge. Critically, in the no-RSI condition, performance under inclusion $(I)$ was similar to performance under exclusion $(E)$ instructions (i.e., $I=E$ ), suggesting that participants had no control over their sequence knowledge, and that the sequence knowledge in the no-RSI condition was fully implicit. (In addition, exclusion performance was above baseline, i.e., $E>B$, indicating that participants in the no-RSI condition were not able to withhold generating parts of the sequence they previously had implicitly learned.) Conversely, in the RSI condition, a robust inclusion-exclusion performance difference (i.e., $I>E$ ) indicated that participants were able to control their sequence knowledge, suggesting that this knowledge is explicit.

\section{Assumptions underlying the PD approach}

These conclusions about the presence versus absence of explicit knowledge, based on comparisons of inclusion and exclusion performance, depend on two assumptions: First, explicit knowledge must be assumed to be fully controllable (otherwise, the lack of an inclusion-exclusion difference cannot be interpreted as the absence of explicit knowledge but may instead reflect uncontrollable explicit knowledge). Put differently, conclusions drawn from the PD approach are limited to controllable explicit knowledge and do not extend to knowledge that may be explicit but not controllable (in the sense that it may be used to affect the similarity of the generated sequence with the learned sequence). This is unproblematic 
as long as the PD approach is used to investigate theories that hold controllability as a central tenet of explicit knowledge. Second, comparisons between inclusion and exclusion task performance are only meaningful if both tasks are indeed comparable measures of sequence knowledge. ${ }^{1}$ In other words, the processes underlying free-generation performance are assumed to be invariant to the inclusion versus exclusion instructions. This assumption is critical for the validity of the PD approach, but it has so far not been tested directly.

The PD generation task has been used repeatedly to investigate sequence learning, but results were typically less clear-cut than those of the initial studies. First, most studies found $I>E$, suggesting the presence of at least some amount of controllable (explicit) knowledge even under no-RSI conditions (Wilkinson \& Shanks, 2004). The debate focused on the evidence for residual implicit knowledge under exclusion instructions: Some studies replicated the $E>B$ finding of Destrebecqz and Cleeremans (2001), and concluded that SRTT learning is driven by implicit knowledge (e.g., Destrebecqz \& Cleeremans, 2003; Q. $\mathrm{Fu}, \mathrm{Fu}, \&$ Dienes, 2008; Haider, Eichler, \& Lange, 2011); other studies found only $E=B$, a pattern interpreted as evidence that only explicit knowledge is acquired during the SRTT (e.g., Destrebecqz, 2004; Norman, Price, \& Duff, 2006; Shanks, Rowland, \& Ranger, 2005; Wilkinson \& Shanks, 2004). Wilkinson and Shanks (2004) failed to replicate the $E>B$ finding and speculated that this may come about because participants attempt to refrain from generating regular sequences under exclusion by resorting to various perseverative response strategies (i.e., by repeatedly generating regular-looking runs such as $1-2-3-4$ ). If participants indeed use different strategies under the inclusion and exclusion instructions, this may violate the invariance assumption.

Moreover, in the presence of explicit knowledge, conclusions about the presence or absence of implicit knowledge, based on comparing exclusion performance with a baseline (i.e., $E>B$ vs. $E=B$ ), depend on additional assumptions regarding the interplay between both types of knowledge. If both types of knowledge may be involved, additional assumptions must be met if one aims at comparing inclusion and exclusion performance across two experimental conditions in order to draw conclusions about the relative contributions of explicit and implicit of knowledge; the ordinal PD approach formulates such a set of assumptions (Hirshman, 2004). Further assumptions are required for a parametric PD measurement model that can provide quantitative estimates of the underlying latent cognitive processes, for instance if the relative magnitude of the effect of a manipulation on explicit versus implicit knowledge is the quantity of interest. We next discuss critical assumptions underlying these two candidate methodological frameworks for the PD paradigm.

The ordinal PD approach. Analyzing their data by comparing inclusion and exclusion performance with a baseline, Destrebecqz and Cleeremans (2001) adopted an analysis strategy that has been later formalized — with modifications - by Hirshman (2004) as the ordinal-PD approach. Instead of providing quantitative estimates of implicit and explicit knowledge, the ordinal-PD approach identified specific patterns of results that allow for ordinal comparisons between two experimental conditions (i.e., conclusions about increasing or decreasing amounts of explicit and/or implicit knowledge). In the light of the then-ongoing controversy about the PD method, this has been critically acclaimed as a way around the strong assumptions underlying the original (parametric) PD (Curran, 2001).

\footnotetext{
${ }^{1}$ For instance, if inclusion and exclusion performance differ in their sensitivity to implicit knowledge, this might lead to an artificial $I>E$ finding suggestive of the presence of explicit knowledge.
} 
However, even this approach is based on assumptions that might be violated in a specific application: First, it is assumed that baseline performance is the same under both inclusion and exclusion instructions - an assumption that may be violated in sequence learning (Stahl, Barth, \& Haider, 2015). Perhaps more critically, the second basic assumption of the ordinal-PD approach holds that both inclusion and exclusion performance are a monotonically increasing function of implicit knowledge; and that inclusion performance monotonically increases but exclusion performance monotonically decreases as a function of explicit knowledge. The exclusion strategies suggested by Wilkinson and Shanks (2004) would, however, imply that explicit knowledge does not necessarily inform exclusion performance: If participants adopted a perseverative response strategy (instead of engaging in an effortful search for their explicit knowledge, and attempting to implement this knowledge into a motor pattern consistent with the exclusion instructions), they would still be able to suppress their exclusion performance to baseline; however, they would not be able to suppress their exclusion performance below baseline (i.e. $E<B){ }^{2}$ The present Experiment 1 provides a first empirical test of this basic assumption of the ordinal-PD approach as applied to the free-generation task. ${ }^{3}$

The parametric PD model. The parametric PD model provides quantitative estimates of the underlying processes but relies on stronger assumptions. This section introduces the parametric PD model and its assumptions and then discusses its relation to the ordinal PD.

The PD model can be formalized as a set of equations describing inclusion $(I)$ and exclusion $(E)$ performance as a function of the probabilities of controlled process, $C$, reflecting explicit knowledge, and the automatic process, $A$, reflecting implicit knowledge, as follows:

$$
I=C+(1-C) * A
$$

and

$$
E=(1-C) * A
$$

These equations reflect the notions that (1) regular transitions generated under inclusion can arise from either the controlled process (with probability $C$ ) or, given that it fails (with probability $1-C$ ), from the automatic process $A$; and (2) regular transitions generated under exclusion are solely due to the automatic process in the absence of the influence of the controlled process, $(1-C) * A$. Solving these equations for $C$ and $A$ (or using parameter estimation techniques for multinomial models) yields estimates of the contributions of the controlled and automatic process.

The validity of the PD method and model has been the target of debate since its introduction by Jacoby (1991; see, e.g., Buchner, Erdfelder, and Vaterrodt-Plünnecke, 1995;

\footnotetext{
${ }^{2}$ Moreover, if response strategies are informed by fragmentary knowledge about the regularity, such fragmentary knowledge might influence exclusion performance in any direction, depending on whether or not the chosen strategy is consistent with what the researcher considers to be successful exclusion. This effect might even outweigh performance changes due to the available explicit knowledge.

${ }^{3}$ Even though Destrebecqz and Cleeremans (2001) deviated from the ordinal PD as put forward by Hirshman (2004), their conclusions still rest on the assumptions of the ordinal PD specified here. Moreover, in order to interpret $I-E$ differences, they implicitly assume that the same strictly monotonic function links automatic and controlled processes with both inclusion and exclusion (whereas Hirshman allowed inclusion and exclusion performance to be linked by different functions). This additional assumption remains untested, yet.
} 
Curran \& Hintzman, 1995; Graf \& Komatsu, 1994). This is because the PD approach is not a theory-free measurement tool but rests on a set of strong and possibly problematic assumptions. First and obviously, it assumes the existence of two qualitatively differentcontrolled and automatic - processes, and it aims to measure the magnitude of their respective contributions. It is, however, not well-suited for comparing single- and dual-process models: To illustrate, Ratcliff, Van Zandt, and McKoon (1995) found that data generated from a singleprocess model could produce a data pattern that, when analyzed using the PD approach, appears to support the existence - and differential contributions - of two qualitatively distinct processes. This implies that empirical dissociations between the controlled and automatic estimates do not necessarily imply the existence of two qualitatively different underlying processes.

Second, it is assumed that both processes operate independently; that is, on each trial, both the explicit and the implicit process attempt to produce a candidate response in parallel, without influencing each other. ${ }^{4}$ In particular, the response proposed by the automatic process is assumed to be uninfluenced by whether the controlled process proposes the same or a different candidate response. Relatedly, the model assumes that independence holds across persons and items; when data are aggregated over (potentially heterogeneous) participants and items, a violation can lead to biases in parameter estimates. There has been considerable debate about the independence assumption in applications of the PD to episodic memory paradigms (Curran \& Hintzman, 1995, 1997; Hintzman \& Curran, 1997; Jacoby \& Shrout, 1997). Evidence suggests that aggregation independence may often be violated; hierarchical extension of the PD model have been proposed to address this problem (Rouder, Lu, Morey, Sun, \& Speckman, 2008).

Third, and most important for the present study, it is assumed that both the controlled and automatic processes are invariant across the inclusion and exclusion instructions. This is reflected in the PD equations by the use of a single parameter $C$ instead of separate parameters for inclusion and exclusion; in other words, the PD equations represent a simplified model that incorporates the invariance assumption $C=C_{\text {Inclusion }}=C_{\text {Exclusion }}$. Similarly, the $\mathrm{PD}$ equations include only a single parameter $A$, reflecting the simplifying assumption that the automatic process is invariant across inclusion and exclusion, $A=A_{\text {Inclusion }}=A_{\text {Exclusion }}$. If the PD instruction affects those cognitive processes, the PD equations do no longer yield valid estimates. Recently, the invariance assumption was indeed found to be violated for the controlled process across three different paradigms (Klauer, Dittrich, Scholtes, \& Voss, 2015). ${ }^{5}$

To summarize, Wilkinson and Shanks (2004) speculated that participants might use perseverative response strategies especially in the exclusion condition of the PD generation task; as a consequence, explicit knowledge would be less likely to affect exclusion performance. In terms of the parametric PD model, this would translate into an invariance violation of the

\footnotetext{
${ }^{4}$ As an alternative to independence, a redundancy relation has been proposed such that the implicit process always operates, whereas the explicit process operates only in a subset of cases (Joordens \& Merikle, 1993). An empirical comparison of the independence and redundancy assumptions has, however, supported independence (Joordens, Wilson, Spalek, \& Paré, 2010).

${ }^{5}$ This assumption has not been tested earlier because the PD equations represent a saturated model: With two data points (i.e., the proportion of correct responses under inclusion and exclusion conditions), only two parameters (i.e., $C$ and $A$ ) can be estimated. An extension of the design is needed to allow for estimating separate parameters $C_{\text {Inclusion }}$ and $C_{\text {Exclusion }}$, and/or $A_{\text {Inclusion }}$ and $A_{\text {Exclusion }}$.
} 
controlled process with $C_{I}>C_{E}$. If the probability of controlled processes in exclusion $C_{E}$ is negligible small, or if the invariance violation increases with increasing explicit knowledge, it cannot be assumed that explicit knowledge reliably decreases with explicit knowledge; thus, in terms of the ordinal-PD approach, an invariance violation of this kind would translate into a violation of the monotonicity assumption. In contrast, if neither is the case (e.g., if the invariance violation remains constant across different levels of explicit knowledge), the monotonicity assumption may hold despite an invariance violation. It is therefore important to test both the monotonicity and the invariance assumptions.

\section{Overview of the present studies}

The present study aimed at testing, in the free-generation task, the assumptions underlying both the ordinal- and the parametric-PD methods. For this purpose, it was necessary to extend the traditional PD design by manipulating both explicit knowledge (in Experiments 1-3) and implicit knowledge (in Experiments 2 \& 3).

We manipulated explicit knowledge by explicitly informing participants, after the SRTT training phase, about a subset of the regular transitions (e.g., 1 out of 6 ) of the sequence. By presenting information about the transitions after training we ensured that the manipulation did not affect the amount of sequence knowledge acquired during training (i.e., we made sure that participants did not use that information during the SRTT to strategically search for more regular transitions). We manipulated implicit knowledge by varying the amount of regularity present in the SRTT training sequence. For this purpose, we used materials with a mere probabilistic regularity; such materials are typically assumed to produce robust implicit knowledge but no explicit knowledge (Jiménez \& Méndez, 1999; Jiménez, Méndez, \& Cleeremans, 1996).

In a test of the monotonicity assumption underlying the ordinal PD approach, Experiment 1 explored the speculation that explicit knowledge remains underutilized in exclusion. To foreshadow, we found that this was indeed the case and that the monotonicity assumption was violated. Results suggested that this is because invariance of the controlled process is violated.

In Experiments 2 and 3, we directly tested the invariance assumptions of the parametric PD model, closely following the methodology used by Klauer et al. (2015): We fit an extended process-dissociation model $\mathcal{M}_{1}$ that allowed for testing the invariance assumption of both the controlled and the automatic process. The model provided us with separate estimates for these processes for both inclusion and exclusion tasks; and we used the differences between these estimates to test the invariance assumption. This model relies on the auxiliary assumptions that each experimental manipulation selectively influenced only one of both processes; these assumptions are tested by goodness-of-fit tests proposed by Klauer (2010). Moreover, in order to justify the auxiliary assumptions, we specified a standard processdissociation model $\mathcal{M}_{2}$ that does not enforce the auxiliary assumptions but enforces the invariance assumption; model comparison techniques (DIC; Spiegelhalter, Best, Carlin, \& Van Der Linde, 2002) were then used to compare model $\mathcal{M}_{1}$ and model $\mathcal{M}_{2}$. If model $\mathcal{M}_{1}$ is favored over model $\mathcal{M}_{2}$, this can be taken as evidence in favor of our auxiliary assumptions over the invariance assumption. Finally, instead of aggregating data, we used hierarchical Bayesian extensions of all models (cf., Klauer, 2010; Rouder \& Lu, 2005; Rouder et al., 
2008). ${ }^{6}$

\section{Experiment 1}

A critical assumption of the ordinal-PD approach is that explicit knowledge monotonically increases inclusion performance and monotonically decreases exclusion performance. If (contrary to this assumption) explicit knowledge does not affect exclusion performance at all, the ordinal PD approach may technically still be applied; however, the results would be misleading if a difference in explicit (but not implicit) knowledge between two conditions led to a difference in inclusion but not in exclusion performance. In this case, the ordinal PD would suggest that the two conditions differ in explicit and implicit knowledge (Hirshman, 2004, Data Pattern I). For the ordinal PD approach to yield valid results, exclusion performance must decline with increasing explicit knowledge, and even fall below baseline when explicit knowledge is sufficiently strong so as to counter the influence of implicit knowledge (this would yield Hirshman's Data Pattern IV, which indicates an increase in explicit knowledge only). Therefore, a critical empirical test for the ordinal-PD approach is whether (and under which conditions) participants are able to use explicit knowledge to suppress generation below baseline levels under exclusion conditions. Our primary goal of Experiment 1 was to test this assumption; therefore, while keeping implicit sequence constant at moderate levels across conditions, we manipulated explicit knowledge by revealing parts of the sequence (i.e., explicit knowledge about 0,1 , or 2 transitions) to participants after finishing the SRTT.

A secondary goal of Experiment 1 was to manipulate the amount of practice participants had with including and excluding their explicit knowledge: If, in a sequence learning study, participants acquired explicit knowledge about a transition during the SRTT, they are likely to encounter the same transition again during the remainder of the SRTT several times: These additional exposures to the transition amount to an opportunity to practice including the explicit knowledge (e.g., intentionally implementing it into a motor pattern). This practice might be essential for the validity of the subsequent PD generation task. Therefore, one might wonder if the explicit sequence knowledge that is acquired during learning is comparable to explicit knowledge via instruction as implemented in our studies. To ensure that the effects of our explicit-knowledge manipulation were comparable with those of acquired knowledge, we amended the generation task by short generation-practice blocks involving inclusion/exclusion instructions that preceded the main inclusion/exclusion blocks. To explore the effects of practice, we manipulated whether a transition was revealed prior to or after these practice blocks: Some transitions were revealed prior to practice, and participants were instructed to implement a motor pattern including (or excluding) the revealed transition. Other transitions were revealed only after practice; for these transitions there was no opportunity to practice.

In five between-subjects conditions, we manipulated both the level of explicit sequence knowledge (i.e., the number of revealed transitions) and-nested within that factor-the amount of generation practice (i.e., whether a transition was revealed prior to or after practice blocks):

1. In the Control group, no explicit knowledge was revealed to participants.

\footnotetext{
${ }^{6}$ This modeling approach controls for interindividual differences and circumvents aggregation artifacts.
} 
2. In the No-Practice group, one transition was revealed immediately before the first main generation block, but after the practice blocks that preceded the first generation block. To avoid carry-over of practice effects from the first generation block, a different non-practiced transition was revealed after the second set of practice blocks and immediately preceding the second main generation block.

3. In the Unspecific-Practice group, one transition was revealed to participants after practice, immediately before each main generation block (as in the No-Practice group). In the third practice block before the exclusion task, participants were asked to inhibit a specific response location (i.e., they were asked not to use the $5^{\text {th }}$ location/ $N$ key).

4. In the Practice group, one transition was revealed to participants immediately before the practice blocks. Participants were encouraged to include (exclude) the revealed transition during generation-practice and in the main generation block.

5. In the Transfer group, information about two transitions was revealed; one of them was non-practiced (as in the No-Practice group), the other one practiced (as in the Practice group). The practiced transition was revealed before the first practice blocks. After these practice blocks, the second (non-practiced) transition was revealed immediately before the first generation block. The practiced transition was again named before participants worked on the practice blocks of the second generation phase. After these practice blocks, a second non-practiced transition was revealed immediately before the second generation block.

This design allowed us to assess overall generation performance for different levels of explicit knowledge. The monotonicity assumption states that with increasing levels of explicit knowledge, the proportion of regular transitions generated under inclusion should increase, while under exclusion it should decrease.

Beyond overall performance, we also analyzed data from specific transitions to test whether explicit knowledge may not be exhaustively expressed during the generation task (and, in particular, under exclusion instructions), and whether the level of its expression depends on generation practice. Our design allowed us to assess generation performance for three main transition types; (1) non-revealed transitions, (2) transitions that were revealed but remained non-practiced, and (3) transitions that were revealed and practiced. ${ }^{7}$

A comparison of non-revealed with (revealed but) non-practiced transitions allows us to assess the degree to which participants can spontaneously (i.e., without practice) make use of their explicit knowledge in the generation task. Comparing non-practiced with practiced transitions allowed us to assess whether transition-specific generation practice could increase the use of explicit knowledge. We also compared whether performance for revealed but non-practiced transitions differs between the No-Practice and Transfer groups, as would be expected if the effect of specific practice transfers to non-practiced explicit knowledge.

\footnotetext{
${ }^{7}$ In the second generation block of the No-Practice, Unspecific-Practice, and Transfer groups, a fourth transition type can be distinguished: Transitions that were revealed but non-practiced before the first generation block. Because participants included (or excluded) these transitions in the previous (i.e., the first) generation block, performance on these transitions should be more similar to practiced than to non-practiced transitions in the second block.
} 
Finally, we explored whether unspecific inhibition practice affects performance for both revealed but non-practiced and/or non-revealed transitions.

In sum, we hypothesized that possessing explicit knowledge may not be sufficient for its expression in the generation task. Specifically, (a) explicit knowledge without practice (No-Practice group) may fail to lead to below-baseline exclusion performance, and (b) this may also hold for non-practiced transitions for participants who practiced another transition (Transfer group). If the exclusion task is not sensitive to manipulations of explicit knowledge, the ordinal PD would yield erroneous conclusions; it would also suggest that the invariance assumption for explicit knowledge of the parametric PD might also be violated. We had no clear hypothesis regarding the unspecific response-inhibition practice, but wanted to explore whether it would be as effective as transition-specific exclusion practice in improving the validity of the generation task as a measure of explicit knowledge.

\section{Method}

Design. The study realized a 5 (Explicit-knowledge-and-Practice: Control, NoPractice, Unspecific-Practice, Practice, Transfer $) \times 2(P D$ instruction: inclusion vs. exclusion $)$ $\times 2$ (Block order: inclusion first vs. exclusion first) design with repeated measures on the $P D$ instruction factor.

Participants. One hundred and forty-seven participants (113 women) aged between 17 and 55 years ( $M=23.7$ years) completed the study. Most were undergraduates from Heinrich-Heine-Universität Düsseldorf. Participants were randomly assigned to experimental conditions. They received either course credit or 3.50 Euro for their participation. ${ }^{8}$

Materials. A probabilistic sequence was generated from the first-order conditional sequence $2-6-5-3-4-1$. With a probability of .6, a stimulus location was followed by the next location from this sequence; otherwise, another stimulus location was randomly chosen from a uniform distribution. There were no direct repetitions of response locations.

Procedure. The experiment consisted of three consecutive parts: Participants first worked on an SRTT (the acquisition task), followed by a generation task and, finally, a debriefing phase. In the acquisition task, all participants performed an SRTT consisting of eight blocks with 144 trials each (for a total of 1,152 responses). SRTT and generation task were run on 17" CRT monitors (with a screen resolution of 1,024 px $\times 768 \mathrm{px}$ ). The viewing distance was approximately $60 \mathrm{~cm}$. A horizontal sequence of six white squares (56 px) was presented on a gray screen. The distance between squares was $112 \mathrm{px}$. Each screen location corresponded to a key on a QWERTZ keyboard (from left to right $\mathrm{Y}, \mathrm{X}$, C, B, N, M). Participants had to respond whenever a square's color changed from white to red by pressing the corresponding key. They were instructed to place the left ring-, middleand index fingers on the keys $\mathrm{Y}, \mathrm{X}$ and $\mathrm{C}$. The right index-, middle- and ring fingers were to be placed on keys B, N and $\mathrm{M}$. There was no time limit for responses in the learning phase (nor in the generation phase). A warning beep indicated an incorrect response. The response-stimulus interval (RSI) was $250 \mathrm{~ms}$; there were no pauses within a single learning block.

Following the SRTT phase, participants were told that stimulus locations during the SRTT followed an underlying sequential structure (but were not informed about the exact

\footnotetext{
${ }^{8}$ The present research used procedures that are exempt from mandatory formal ethical approval under the ethical guidelines of the Deutsche Gesellschaft für Psychologie.
} 
sequence). They were then asked to try to generate a short sequence of six locations that followed this structure.

The generation task followed, consisting of two main generation blocks of 120 responses that were each preceded by three generation-practice blocks of twelve responses. Before entering practice blocks, one transition was revealed to participants in the Practice and Transfer groups. After practice blocks, another transition was revealed to participants in the No-Practice, Unspecific-Practice, and Transfer groups. Participants were told to memorize those transitions and to use their knowledge in all following tasks.

The main inclusion block was preceded by three practice blocks that were all performed under inclusion instructions. The main exclusion block was preceded by two practice blocks that were performed under inclusion instructions and a third practice block that was performed under exclusion instructions. The first two practice blocks (those that always involved inclusion instructions) were aimed at allowing participants to integrate their acquired sequence knowledge with just-revealed sequence information. The third block (that involved either inclusion or exclusion instructions, depending on the instructions of the subsequent main generation block) was aimed at allowing participants to familiarize with inclusion/exclusion instructions. ${ }^{9}$

In both main generation and generation-practice blocks, under inclusion (exclusion) instructions, participants were told to generate a sequence as similar (dissimilar) as possible to the sequence from the acquisition task. Participants were instructed to follow their intuition if they had no explicit knowledge about the underlying sequence. Participants who had received information about transitions were instructed to include (exclude) the revealed transitions. Question marks appeared at all locations and participants' key presses were reflected by the corresponding square's color changing to red. Direct repetitions were explicitly discouraged and were followed by a warning beep.

Upon completing the computerized task, participants were asked to complete a questionnaire containing the following items (translated from German): (1) "One of the tasks mentioned a sequence in which the squares lit up during the first part of the study. In one of the experimental conditions, the squares did indeed follow a specific sequence. Do you think you were in this condition or not?", (2) "How confident are you (in \%)?", and (3) "Can you describe the sequence in detail?". Subsequently, participants were asked to indicate, for each of the six response keys, the next key in the sequence on a printed keyboard layout and to indicate how confident they were in this decision. Finally, participants were thanked and debriefed.

Data analysis. All analyses were performed using the $\mathrm{R}$ software ${ }^{10}$ and Stan (Carpenter et al., 2016). For analyses of reaction times during the acquisition task, we

\footnotetext{
${ }^{9}$ In Experiment 1, we held constant the number of generation-practice blocks involving inclusion/exclusion instructions. We based the number of presented practice blocks on our observations in Experiment 2 (that was conducted earlier): In Experiment 2, participants worked on practice blocks until they had consistently included/excluded a revealed transition. Prior to main inclusion blocks, participants in Experiment 2 needed $M=2.98, M d=2$ inclusion practice blocks. Prior to main exclusion blocks, participants in Experiment 2 needed $M=1.26, M d=1$ exclusion and $M=1.52, M d=1$ inclusion practice blocks. This suggests that the choice of 3 practice blocks - 3 under inclusion instructions (for the inclusion block), or 2 under inclusion and 1 under exclusion instructions (for the exclusion block) — should be sufficient for the majority of participants.

${ }^{10}$ We used R (Version 3.4.3; R Core Team, 2017) and the R-packages afex (Version 0.19.1; Singmann, Bolker, Westfall, \& Aust, 2018), and papaja (Version 0.1.0.9709; Aust \& Barth, 2018).
} 
excluded the first trial of each block as well as trials with errors, trials succeeding an error, reactions faster than $50 \mathrm{~ms}$ and those slower than 1,000 ms. For analyses of error rates during the acquisition task, we excluded the first trial of each block.

Generation task analyses were conducted with the first trial of each block as well as any response repetitions excluded. During the generation task, participants generated 120 keypresses. We coded these data as 119 first-order conditional transitions (e.g., a 4-key sequence $1-2-3-4$ was coded as the three transitions $1-2,2-3$, and 3-4); we then computed the frequency of transitions that were consistent (i.e., part of) or inconsistent with (i.e., not part of) the training sequence. This scoring procedure follows the one used in the studies of Destrebecqz and Cleeremans (2001) and Wilkinson and Shanks (2004). ${ }^{11}$ Response repetitions were excluded from analyses, as these were explicitly discouraged in the instructions. For repeated-measures ANOVAs, Greenhouse-Geisser-corrected degrees of freedom are reported.

\section{Results}

We first analyzed the performance data from the SRTT to determine whether sequence knowledge had been acquired during the task. Next, we analyzed generation task performance using an ordinal-PD approach (full descriptive statistics and additional model-based analyses are reported in Appendices A and C). Finally, to test our predictions regarding the different effects of practice, we analyzed generation performance for transitions about which explicit knowledge had been revealed.

Acquisition task. If participants acquired knowledge about the regularity underlying the sequence of key presses, we expect a performance advantage for regular over irregular transitions, reflected in reduced RT and/or error rate. If this advantage is due to learning, it is expected to increase over SRTT blocks.

Reaction times. Figure 1 shows reaction times during acquisition. We conducted an 8 (Block number $) \times 2$ (FOC transition status: regular vs. irregular) repeated-measures ANOVA. There was a main effect of block number, $F(4.05,591.75)=80.42, M S E=1,658.05$, $p<.001, \hat{\eta}_{G}^{2}=.048$, with RT decreasing over blocks. There also was a main effect of FOC transitions status, $F(1,146)=716.67, M S E=982.05, p<.001, \hat{\eta}_{G}^{2}=.062$, reflecting faster responses to regular than to irregular transitions. The interaction of block number and FOC transition status was also significant, $F(6.39,933.34)=45.89, M S E=257.06, p<.001$, $\hat{\eta}_{G}^{2}=.007$, reflecting the finding that the RT advantage for regular transitions increased over blocks, which indicated successful sequence learning.

Error rates. Figure 2 shows error rates during acquisition. The pattern of findings was similar to that obtained for RT. We conducted an 8 (Block number $) \times 2$ (FOC transition status: regular vs. irregular) repeated-measures ANOVA that revealed a main effect of block number, $F(6.29,917.65)=8.35, M S E=9.42, p<.001, \hat{\eta}_{G}^{2}=.015$, reflecting increasing

\footnotetext{
${ }^{11}$ This scoring procedure ignores sequential dependencies inherent in the free-generation data. For instance, the frequency with which a specific location is generated determines how often a transition starting from this location can be generated, and thereby, how well the knowledge available about this transition can be estimated: To illustrate, if the starting point of a transition is never generated, it is not possible to learn anything about the knowledge participants may have acquired about this transition. We believe this is not a serious threat to the present analysis because participants generated the locations at comparable rates. Still, other types of dependencies may yet turn out to be more problematic, and future research should consider modeling entire generation sequences instead of individual transitions.
} 


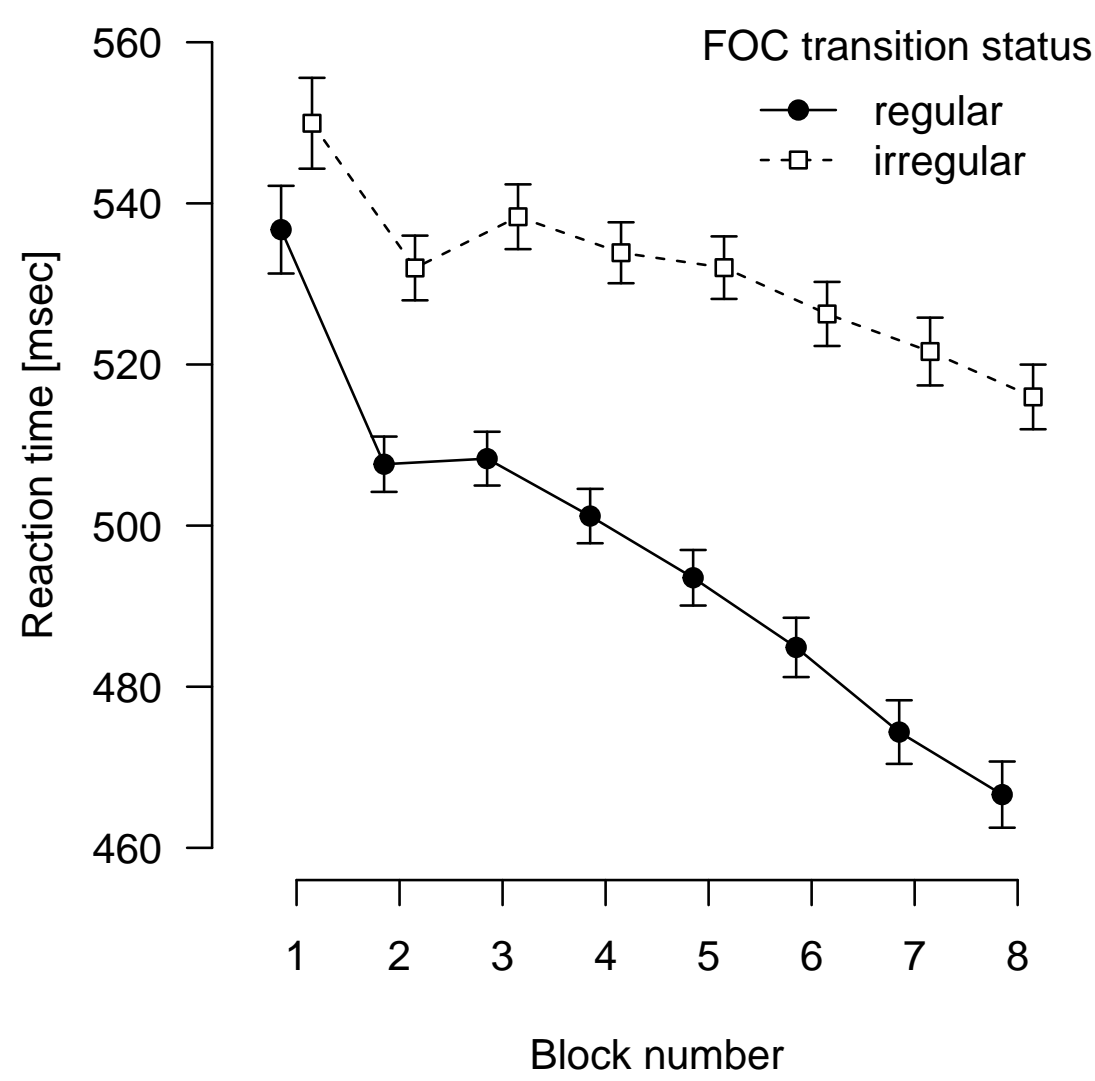

Figure 1. RTs during acquisition phase of Experiment 1, split by FOC transition status. Error bars represent $95 \%$ within-subjects confidence intervals.

error rates over blocks; and a main effect of FOC transition status, $F(1,146)=188.88$, $M S E=11.92, p<.001, \hat{\eta}_{G}^{2}=.066$, reflecting an accuracy advantage (i.e., lower error rates) for regular transitions. The interaction of block number and FOC transition status was also significant, $F(6.53,953.88)=7.36, M S E=7.09, p<.001, \hat{\eta}_{G}^{2}=.011$, reflecting an increase of the accuracy advantage for regular (as compared to irregular) transitions over blocks, indicating successful sequence learning.

Generation task. We first analyzed generation performance by applying standard ANOVA techniques to the proportions of regular transitions generated in inclusion and exclusion blocks. We then analyzed generation performance for those transitions that were revealed to participants, testing our hypotheses about the effects of practice on generation performance.

Overall generation performance. Figure 3 shows the overall generation performance. We conducted a 5 (Explicit-knowledge-and-Practice: Control vs. No-Practice vs. Unspecific-Practice vs. Practice vs. Transfer $) \times 2($ Order: Inclusion first vs. Exclusion first $) \times 2(P D$ instruction: Inclusion vs. Exclusion $)$ ANOVA that revealed a main effect of $P D$ instruction, $F(1,137)=64.03, M S E=176.93, p<.001, \hat{\eta}_{G}^{2}=.199$, participants generated more regular transitions in inclusion than exclusion blocks; and a main effect of Explicitknowledge-and-Practice, $F(4,137)=13.81, M S E=155.01, p<.001, \hat{\eta}_{G}^{2}=.158$, indicating a clear influence of our manipulation of explicit knowledge and on generation performance. 


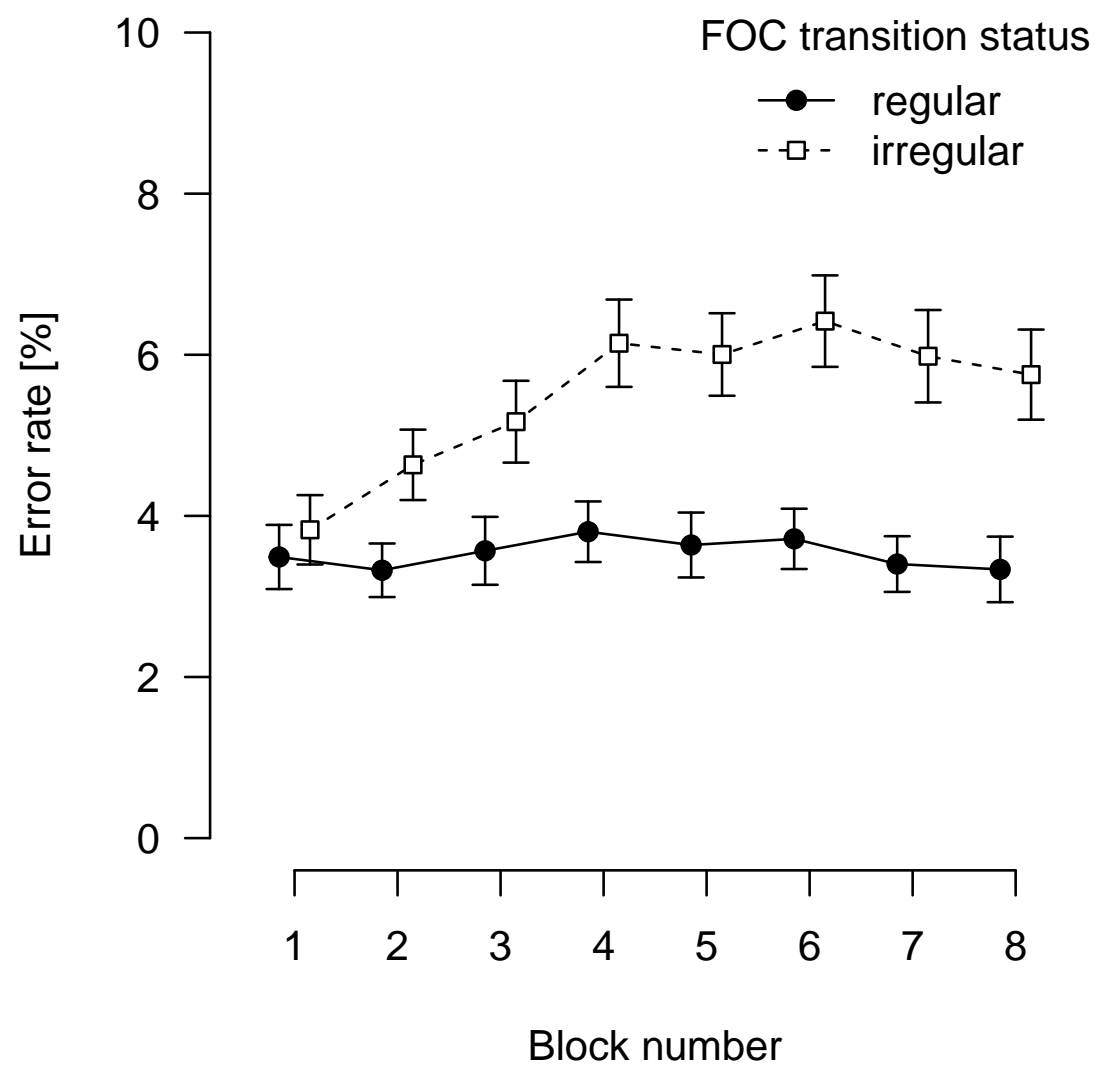

Figure 2. Error rates during acquisition phase of Experiment 1, split by FOC transition status. Error bars represent $95 \%$ within-subjects confidence intervals.

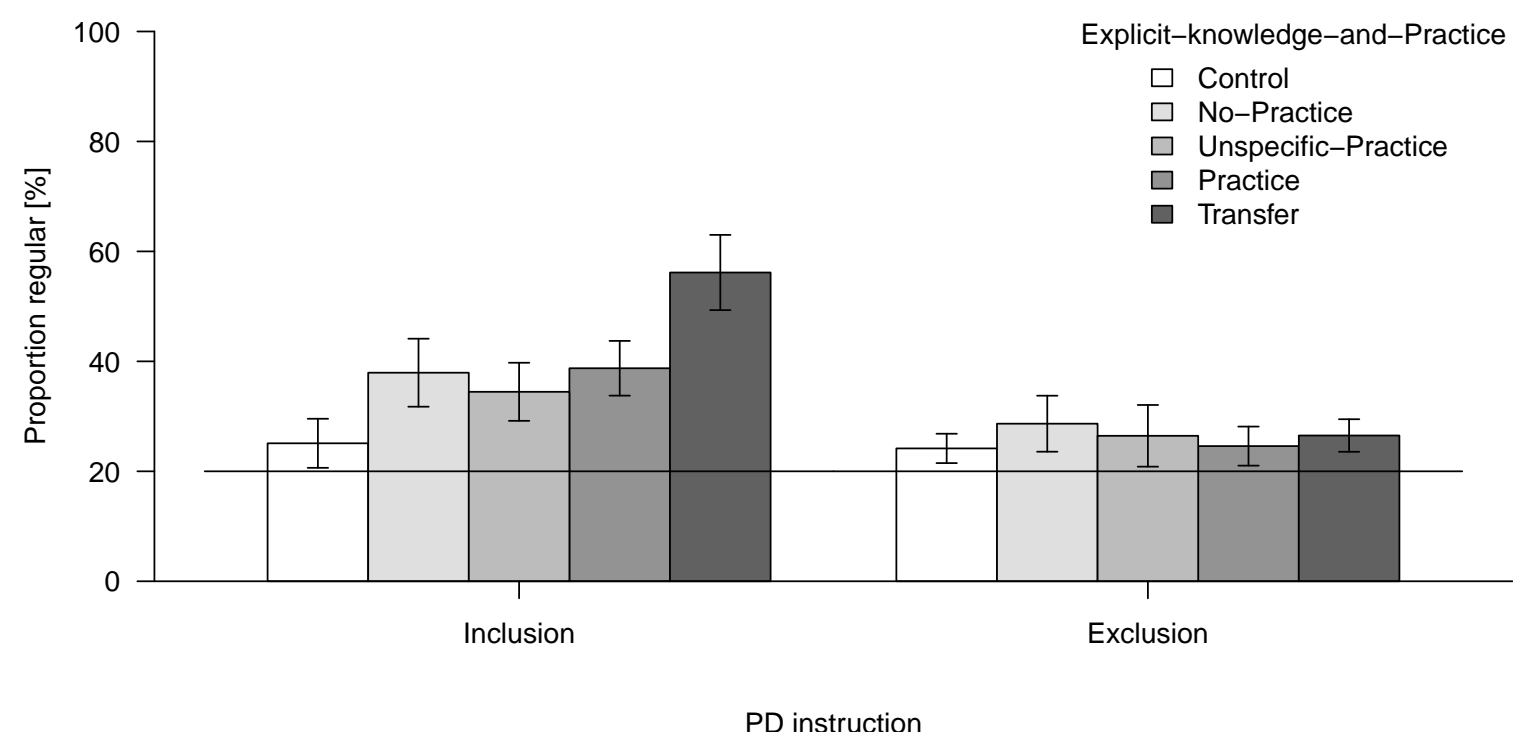

Figure 3. Generation performance in Experiment 1, excluding repetitions. Error bars represent $95 \%$ confidence intervals. Horizontal lines represent chance baseline. 


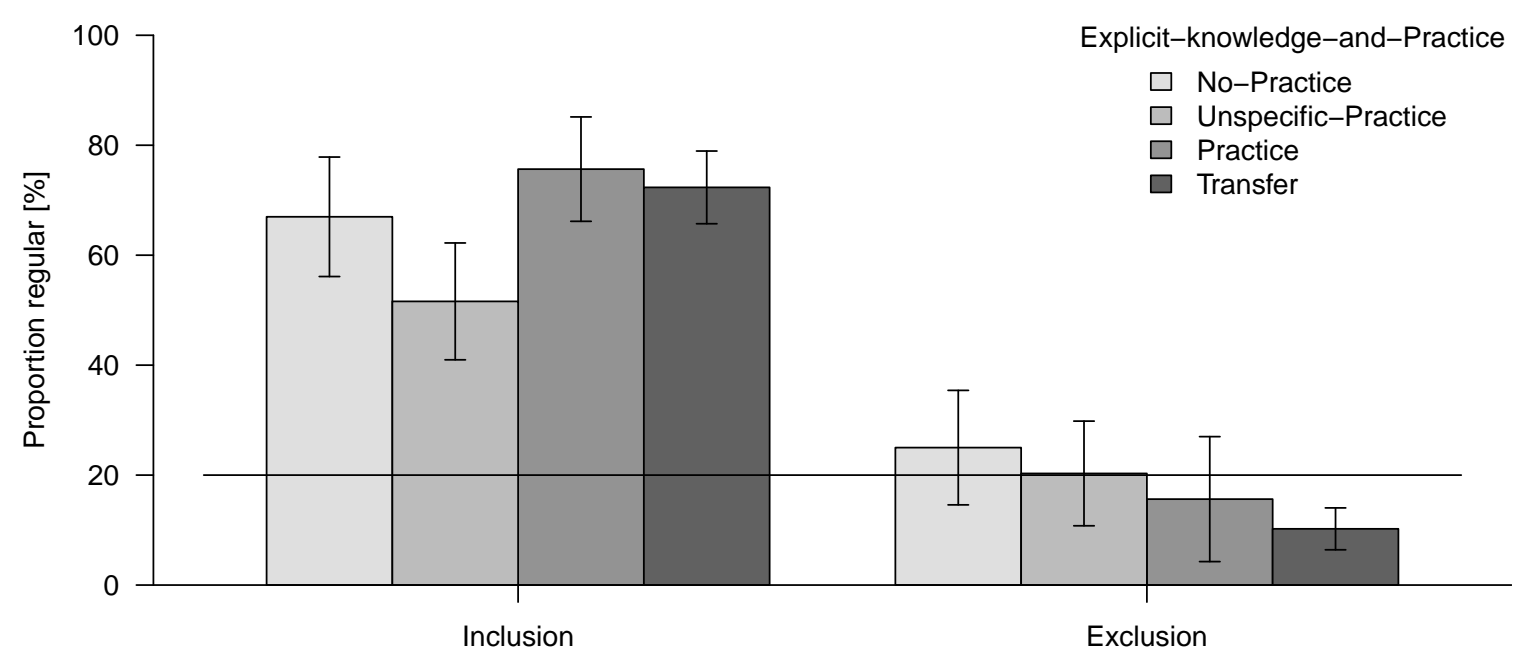

PD instruction

Figure 4. Generation performance for revealed transitions in Experiment 1. Error bars represent $95 \%$ confidence intervals. Horizontal lines represent chance baseline.

Moreover, the interaction of Explicit-knowledge-and-Practice and PD instruction reached significance, $F(4,137)=9.63, M S E=176.93, p<.001, \hat{\eta}_{G}^{2}=.130$, indicating that the effect of Explicit-knowledge-and-Practice is qualified by $P D$ instruction. The interaction of $P D$ instruction and block order also reached significance, $F(1,137)=10.89, M S E=176.93$, $p=.001, \hat{\eta}_{G}^{2}=.041$. To disentangle these interactions, we analyzed inclusion and exclusion performance, separately.

Analyzing inclusion blocks, a 5 (Explicit-knowledge-and-Practice: Control vs. NoPractice vs. Unspecific-Practice vs. Practice vs. Transfer) $\times 2$ (Order: Inclusion first vs. Exclusion first) ANOVA revealed a significant main effect of Explicit-knowledge-andPractice, $F(4,137)=17.74, M S E=211.85, p<.001, \hat{\eta}_{G}^{2}=.341$, indicating that inclusion performance increased with the number of revealed transitions; and a main effect of block order, $F(1,137)=9.95, M S E=211.85, p=.002, \hat{\eta}_{G}^{2}=.068$ : participants generated more regular transitions if inclusion followed exclusion; the interaction of Explicit-knowledgeand-Practice and block order did not reach significance, $F(4,137)=0.52, M S E=211.85$, $p=.723, \hat{\eta}_{G}^{2}=.015$.

Analyzing exclusion blocks, a 5 (Explicit-knowledge-and-Practice: Control vs. NoPractice vs. Unspecific-Practice vs. Practice vs. Transfer) $\times 2$ (Order: Inclusion first vs. Exclusion first) ANOVA revealed no significant effects on exclusion performance (all $p \mathrm{~s} \geq .143)$. Specifically, increasing levels of explicit knowledge did not reduce the level of regular transitions generated under exclusion instructions.

Generation performance for revealed transitions. To explore effects of practice, we analyzed generation performance for only those transitions about which explicit knowledge was revealed (see Figure 4). A 4 (Explicit-knowledge-and-Practice: No-Practice vs. Unspecific-Practice vs. Practice vs. Transfer $) \times 2($ Order: Inclusion first vs. Exclusion first $) \times 2(P D$ instruction: Inclusion vs. Exclusion $)$ ANOVA revealed a nonsignificant main effect of Explicit-knowledge-and-Practice, $F(3,110)=2.00, M S E=660.29, p=.119$, 
Practice vs. No-Practice groups

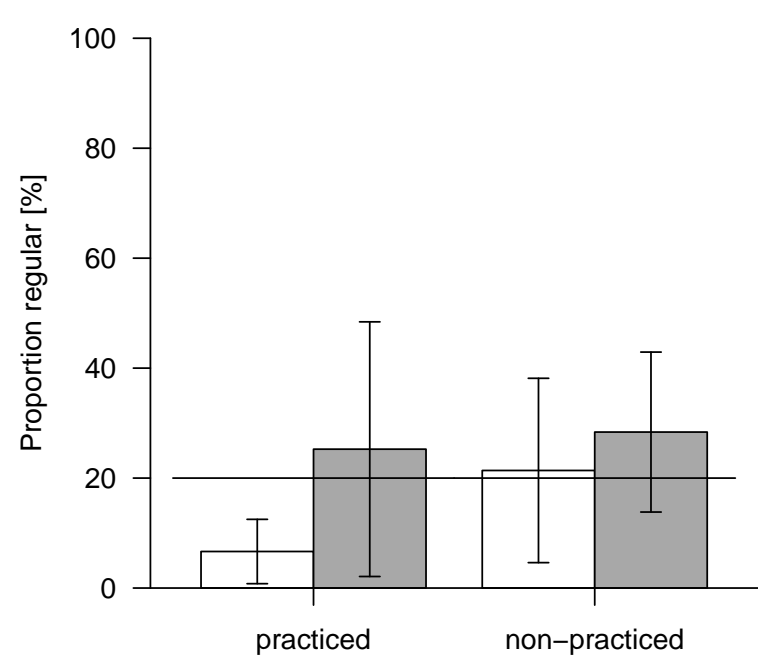

Transition type
Transfer group

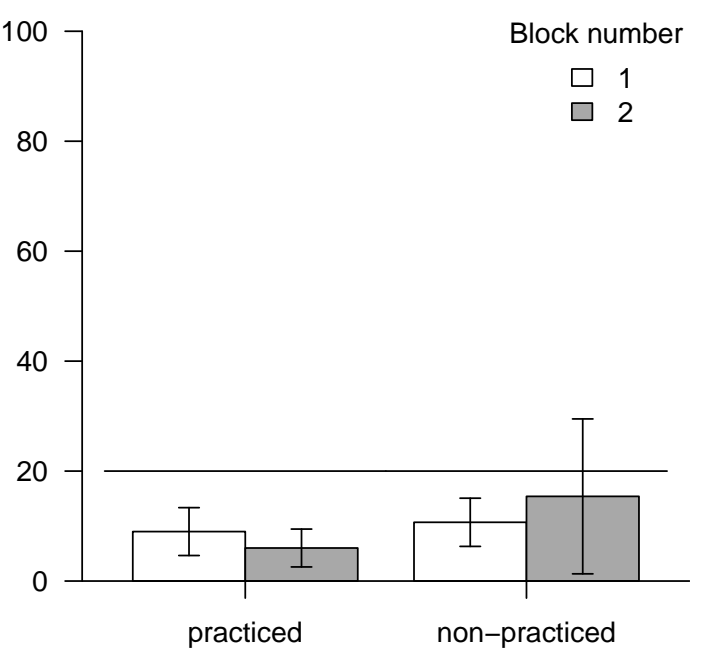

Transition type

Figure 5. Exclusion performance for revealed transitions in Experiment 1. Left: Betweensubjects comparison between Practice and No-Practice groups. Right: Within-subjects comparison in Transfer group. Horizontal lines represent chance baseline.

$\hat{\eta}_{G}^{2}=.028$, but a significant main effect of $P D$ instruction, $F(1,110)=243.88, M S E=575.67$, $p<.001, \hat{\eta}_{G}^{2}=.508$, and their significant interaction, $F(3,110)=5.59, M S E=575.67$, $p=.001, \hat{\eta}_{G}^{2}=.066$. The main effect of $P D$ instruction reflects the clear influence of the PD instruction on the expression of explicit knowledge depicted in Figure 4. It is present in all practice conditions but modulated by amount of knowledge and type of practice (i.e., greater effects given specific practice): The effect was greatest in the Transfer group, $t(29)=-14.84$, $p<.001, d=-2.71$; somewhat smaller in the Practice group, $t(28)=-9.79, p<.001$, $d=-1.82$; it was still smaller and comparable in the No-practice group, $t(28)=-5.25$, $p<.001, d=-0.97$, and the Unspecific-practice group, $t(29)=-5.13, p<.001, d=-0.94$.

We investigated this issue more closely in two sets of follow-up analyses. Whereas the above findings suggest that practice improves the degree to which explicit knowledge is expressed in the generation task, it does not elucidate the mechanism by which this occurs. One mechanism by which practice may improve performance is by boosting the proportion of regular transitions in inclusion blocks.

Inclusion performance for revealed transitions in the No-Practice and Practice groups was analyzed as a function of practice (practiced vs. non-practiced) . Results showed no effect of practice on inclusion performance, $F(1,56)=0.21, M S E=696.48, p=.652$, $\hat{\eta}_{G}^{2}=.004$. Similarly, when we compared inclusion performance for practiced vs. nonpracticed transitions in the Transfer group, there was no effect of practice, $F(1,29)=1.19$, $M S E=365.77, p=.285, \hat{\eta}_{G}^{2}=.014$. We conclude that practice did not affect inclusion performance for revealed transitions.

Next, we analyzed whether practice improves suppressing the regular transition in the exclusion task. We speculated that, without training, participants might not be able to suppress their generation of regular transitions below baseline level in the exclusion task. We 
compared generation performance for the revealed transitions between the No-Practice and Practice groups (see Figure 5, left panel). The expected below-baseline performance was not found when aggregating across both blocks: Whereas the direction of effects was as expected, there was no deviation from chance, neither for the practice condition, $t(28)=-0.79$, $p=.219, d=-0.15$, nor for the no-practice condition, $t(28)=1.60, p=.940, d=0.30$. However, the pattern was present in the first block: Below-chance performance was found for the practice condition, $t(14)=-4.89, p<.001, d=-1.26$, but not for the no-practice condition, $t(13)=0.18, p=.569, d=0.05$.

To more directly establish a practice effect, we next turned to the data from the Transfer group for a within-subjects comparison of practiced and non-practiced transitions. In doing so, we also addressed the transfer hypothesis: If training on one transition transfers to other transitions, we should find below-chance performance also for non-practiced transitions in the Transfer group. This was confirmed: Generation performance was below baseline both for practiced, $t(29)=-9.60, p<.001, d=-1.75$ and non-practiced transitions, $t(29)=-2.04, p=.025, d=-0.37$, indicating transfer of exclusion practice from practiced to non-practiced transitions (see Figure 5, right panel). ${ }^{12}$

\section{Discussion}

Participants in Experiment 1 acquired knowledge about the sequence, as expressed in RT and accuracy advantages for regular transitions that increased over SRTT blocks. Participants received different amounts of instructed explicit knowledge, and they were able to express this knowledge in the inclusion task, as revealed by a main effect of Explicitknowledge-and-Practice on inclusion performance. Conversely, participants were not able to express their knowledge in the exclusion task, as there was no effect of our explicit knowledge manipulation on exclusion performance. This finding violates the monotonicity assumption.

Analyzing exclusion performance of only revealed transitions, performance differed across groups (i.e., practice conditions), suggesting that explicit knowledge was indeed expressed under exclusion instructions, and that specific exclusion practice was beneficial to implementing these instructions. However, even with practice, inclusion performance did not reach ceiling and exclusion performance did not reach floor levels, indicating that participants were not able to exhaustively express their explicit knowledge of these transitions in the generation task. This pattern of results is also in line with Wilkinson and Shanks's (2004) speculation that participants adopt perseverative response strategies especially under exclusion instructions; these might be mildly informed by strong explicit knowledge (e.g., in our Transfer group).

Importantly, the results showed no effect of practice on inclusion performance of revealed transitions. Such an effect would be expected if explicit knowledge revealed to participants after the end of the SRTT differed from explicit knowledge acquired by participants during the SRTT (e.g., because in the latter case, during the remainder of the SRTT participants would have repeated opportunities to practice including their explicit knowledge by intentionally implementing it into a motor pattern). The absence of this effect corroborates the validity of the present explicit-knowledge manipulation.

\footnotetext{
${ }^{12}$ Analyzing only the first block revealed the same pattern of results: Generation performance was below chance for practiced, $t(14)=-5.42, p<.001, d=-1.40$, as well as for non-practiced transitions, $t(14)=-4.56, p<.001, d=-1.18$.
} 
Furthermore, even if (after repeated opportunity to practice) participants were able to refrain from generating some of the revealed transitions, this was not consistently reflected in below-baseline overall generation performance. It can thus be concluded that increasing amounts of explicit knowledge do not necessarily lead to fewer regular transitions being generated; the monotonicity assumption of the ordinal PD is thus violated. As a consequence, if the ordinal PD were applied to such data, a change in only explicit knowledge between two conditions would thus be misinterpreted as reflecting changes in both implicit and explicit knowledge.

In sum, Experiment 1 showed that, first, increasing amounts of explicit knowledge were not reflected in decreasing levels of exclusion performance, showing that the monotonicity assumption underlying the ordinal PD approach is violated. Second, explicit knowledge can nevertheless be used under exclusion instructions to decrease performance to below-baseline levels (if not exhaustively, and only under specific practice conditions); thus, we can reject the hypothesis that explicit knowledge does not affect exclusion performance at all.

Third, the usage of explicit knowledge in the generation task was higher under inclusion than exclusion, suggesting a violation of invariance (i.e., $C_{I}>C_{E}$ ). Experiments 2 and 3 more directly tested this assumption.

\section{Experiment 2}

Experiment 2 applied the parametric PD model and tested the invariance assumption for automatic and controlled processes using materials with first-order conditional regularities. We implemented two different levels of implicit knowledge by presenting either random or probabilistic sequences to participants during the SRTT. Orthogonally, we implemented two different levels of explicit knowledge by experimentally inducing such knowledge: After the SRTT, we informed one half of participants about one of the six transitions in the regular sequence.

\section{Method}

Design. The study realized a 2 (Material: random vs. probabilistic) $\times 2$ (Explicit knowledge: no transition revealed vs. one transition revealed $) \times 2(P D$ instruction: inclusion vs. exclusion $) \times 2$ (Block order: inclusion first vs. exclusion first) design with repeated measures on the $P D$ instruction factor.

Participants. One hundred and twenty-one participants ( 87 women) aged between 17 and 51 years ( $M=23.7$ years) completed the study. Most were undergraduates from University of Cologne. Participants were randomly assigned to experimental conditions. They received either course credit or 3.50 Euro for their participation.

Materials. We used two different types of material:

- A random sequence was randomly generated for each participant anew by drawing with replacement from a uniform distribution of six response locations.

- A probabilistic sequence was generated similar to the sequence in Experiment 1.

In both materials there were no direct repetitions of response locations. In the random group, there was no "regular" sequence, and transition frequencies varied across persons. To compute the dependent variable in the generation task (i.e., the proportion of rule-adhering 
or regular transitions), we used the generating sequence for participants who worked on probabilistic material; for participants who worked on random material, we determined an individual criterion for each participant. In order to calculate the individual criteria, we first generated all possible sequences that follow the constraints that they are 6-item-sequences that do not contain repetitions and contain all six response locations. Then, for each participant, we calculated how many of the transitions that were presented during the acquisition phase followed each of those 120 non-redundant 6 -item-sequences. We then chose, for each participant anew, the sequence that most frequently adhered to the transitions presented during acquisition phase and took this 6-item-sequence to calculate the dependent variable during the generation phase. Given probabilistic materials, this scoring leads to the same results as using the generating sequence as a criterion. For the group that was instructed about a regular transition, the criterion sequence always contained the revealed transition.

Procedure. During an SRTT consisting of eight blocks with 144 trials each (for a total of 1,152 responses), participants were trained on either random or probabilistic sequences. After the SRTT, participants were informed about the underlying sequential structure of stimulus locations and asked to generate a short sequence of six key presses that followed this (unspecified) structure.

The generation task followed, with counterbalanced order of inclusion versus exclusion blocks. Prior to the inclusion task, two generation-practice blocks involved inclusion instructions; prior to the exclusion task, the first generation-practice block was performed under inclusion instructions and the second generation-practice block was performed under exclusion instructions. If participants who were explicitly informed about one transition failed to include (exclude) the revealed transition in practice blocks, they were informed that they did something wrong; the already revealed transition was again presented and two additional practice blocks had to be performed (if a participant failed to include the transition during the first practice block, they were immediately presented with the sequence knowledge, again). This procedure was repeated until the revealed transition was successfully included (excluded) in two consecutive practice blocks (in contrast to Experiment 1, where the number of practice blocks was held constant). Upon completing the computerized task, participants answered the same questionnaire as in Experiment 1.

Data analysis. For analyses of reaction times during the acquisition task, we excluded the first trial of each block as well as trials with errors, trials succeeding an error, reactions faster than $50 \mathrm{~ms}$ and those slower than 1,000 ms. For analyses of error rates during the acquisition task, we excluded the first trial of each block.

Generation task analyses were conducted with the first trial of a block as well as any response repetitions excluded. For the model-based analyses, we used hierarchical Bayesian extensions of the process-dissociation model (Klauer, 2010; Rouder \& Lu, 2005; Rouder et al., 2008). We estimated model $\mathcal{M}_{1}$ that extended the traditional process-dissociation model by allowing for a violation of the invariance assumption: Controlled and automatic processes were allowed to vary as a function of instruction (inclusion vs. exclusion). The first-level equations of this model were given by:

$$
\begin{aligned}
I_{i j} & =C_{i j m}+\left(1-C_{i j m}\right) A_{i j m}, & & m=1 \\
E_{i j} & =\left(1-C_{i j m}\right) A_{i j m}, & & m=2
\end{aligned}
$$


where $i$ indexes participants, $j$ indexes transition type (i.e., revealed: $j=1$; nonrevealed: $j=2$ ), and $m$ indexes the $P D$ instruction condition (inclusion: $m=1$; exclusion: $m=2$ ).

Parameters $C_{i j m}$ and $A_{i j m}$ are probabilities in the range between zero and one; following previous work (e.g. Albert \& Chib, 1993; Klauer et al., 2015; Rouder et al., 2008), we used a probit function to link these probabilities to the second-level parameters as follows:

$$
C_{i j m}= \begin{cases}\Phi\left(\mu_{k m}^{(C)}+\delta_{i m}^{(C)}\right) & \text { if } j=1 \text { (item has been revealed) } \\ 0 & \text { if } j=2 \text { (item has not been revealed) }\end{cases}
$$

and

$$
A_{i j m}=\Phi\left(\mu_{j k m}^{(A)}+\delta_{i j m}^{(A)}\right)
$$

where $\Phi$ denotes the standard normal cumulative distribution function, $\mu_{k m}^{(C)}$ is the fixed effect of material $k$ (that participant $i$ worked on during the SRTT) and PD instruction condition $m$ on controlled processes. $\delta_{i m}^{(C)}$ is the $i$ th participant's deviation from his or her group's mean.

Accordingly, $\mu_{j k m}^{(A)}$ is the fixed effect of transition type $j$, material $k$, and $P D$ instruction condition $m$ on automatic processes, and $\delta_{i j m}^{(A)}$ is the $i$ th participant's deviation from the corresponding mean. Priors on parameters are given in the Appendix D.

This specification imposes two auxiliary assumptions to the model: First, it is assumed that controlled processes $C$ are set to zero for nonrevealed transitions (i.e., $C=0$ for $j=2$ ), in other words, we assumed that no explicit knowledge has been acquired during the SRT phase. Second, it is assumed that automatic processes $A$ do not vary as a function of the between-subjects manipulation of explicit knowledge $l$ (i.e., $\mu_{l=1}^{(A)}=\mu_{l=2}^{(A)}$ ). These assumptions allowed us to relax and test the invariance assumption by obtaining separate estimates of both $C$ and $A$ for the inclusion and exclusion conditions (note that a full model relaxing all three assumptions cannot be estimated).

To assess goodness of fit, we used posterior predictive model checks as proposed by Klauer (2010): Statistic $T_{A 1}$ summarizes how well the model describes the individual category counts for the eight categories (revealed vs. nonrevealed $\times$ regular vs. nonregular $\times$ inclusion vs. exclusion). Statistic $T_{B 1}$ summarizes how well the model describes the covariations in the data across participants.

Additionally, we also estimated a model $\mathcal{M}_{2}$ that does not impose the auxiliary assumptions but enforces the invariance assumptions (i.e., parameters were not allowed to vary as a function of $\mathrm{PD}$ instruction condition $m$ ):

$$
\begin{aligned}
I_{i j} & =C_{i j}+\left(1-C_{i j}\right) A_{i j} \\
E_{i j} & =\left(1-C_{i j}\right) A_{i j}
\end{aligned}
$$

The second-level equations of model $\mathcal{M}_{2}$ are then given by:

$$
C_{i j}=\Phi\left(\mu_{j k l}^{(C)}+\delta_{i j}^{(C)}\right)
$$

and

$$
A_{i j}=\Phi\left(\mu_{j k l}^{(A)}+\delta_{i j}^{(A)}\right)
$$


where $i$ indexes participants, $j$ indexes transition type, $k$ indexes the learning material that participant $i$ worked on during the SRTT, and $l$ indexes the manipulation of explicit knowledge (i.e., whether or not a transition has been revealed to participant $i$ ). Note that, given this model specification, separate parameters are estimated for each between-subjects condition $k l$ and each transition type $j$, while the invariance assumption is maintained (i.e., there is no index $m$ for $P D$ instruction in the model equations).

These two models were compared using the deviance information criterion DIC (Spiegelhalter et al., 2002; Spiegelhalter, Best, Carlin, \& van der Linde, 2014); the DIC is an extension of AIC for Bayesian hierarchical models, and differences of 10 are considered to imply strong evidence in favor of the model with the lower DIC value (Klauer et al., 2015). Therefore, if model $\mathcal{M}_{1}$ outperforms model $\mathcal{M}_{2}$, it can be concluded that the auxiliary assumptions are less problematic than the invariance assumptions.

Furthermore, model $\mathcal{M}_{1}$ yields separate estimates of controlled and automatic processes for both inclusion and exclusion. The invariance assumption can be targeted directly by calculating the posterior differences $A_{I}-A_{E}$ and $C_{I}-C_{E}$ : If the posterior distributions of these differences include zero, it can be concluded that the respective invariance assumption holds; if the posterior does not contain zero, it can be concluded that the respective invariance assumption is violated.

\section{Results}

We first analyzed the performance data from the SRTT to determine whether sequence knowledge had been acquired during the task. Next, we analyzed generation task performance using hierarchical PD models (descriptive statistics and ordinal-PD analyses are reported in Appendices A and B).

Acquisition task. If participants acquired knowledge about the (probabilistic) regularity underlying the sequence of key presses, we expect a performance advantage for regular over irregular transitions, reflected in reduced RT and/or error rate. If this advantage is due to learning, it is expected to increase over SRTT blocks.

Reaction times. Figure 6 shows reaction times during the SRTT. We conducted a 2 (Material: Random vs. Probabilistic) $\times 8$ (Block number $) \times 2($ FOC transition status: regular vs. irregular) ANOVA that revealed a main effect of material, $F(1,119)=8.11$, $M S E=39,617.25, p=.005, \hat{\eta}_{G}^{2}=.055$; a main effect of block number $F(4.89,582.06)=$ $33.35, M S E=1,032.91, p<.001, \hat{\eta}_{G}^{2}=.029$; a main effect of FOC transition status, $F(1,119)=125.46, M S E=714.88, p<.001, \hat{\eta}_{G}^{2}=.016$; an interaction of material and FOC transition status, $F(1,119)=121.57, M S E=714.88, p<.001, \hat{\eta}_{G}^{2}=.015$; an interaction of block number and FOC transition status, $F(6.32,752.52)=10.68, M S E=197.96, p<.001$, $\hat{\eta}_{G}^{2}=.002$; and a three-way interaction between material, block number, and FOC transition status, $F(6.32,752.52)=5.70, M S E=197.96, p<.001, \hat{\eta}_{G}^{2}=.001$.

Separate ANOVAs for each material condition yielded, for random material, only a significant main effect of block number, $F(4.38,258.47)=13.09, M S E=1,276.78, p<.001$, $\hat{\eta}_{G}^{2}=.026$, with RTs decreasing over blocks (all other $F_{\mathrm{s}}<1$ ). For probabilistic material, in contrast, we obtained main effects of block number, $F(5.07,304.28)=22.09, M S E=$ 891.30, $p<.001, \hat{\eta}_{G}^{2}=.035$; and of transition status, $F(1,60)=182.32, M S E=976.60$,

$p<.001, \hat{\eta}_{G}^{2}=.061$ (i.e. responses to regular transitions were faster than those for irregular transitions); importantly, we also obtained an interaction of block number and transition 

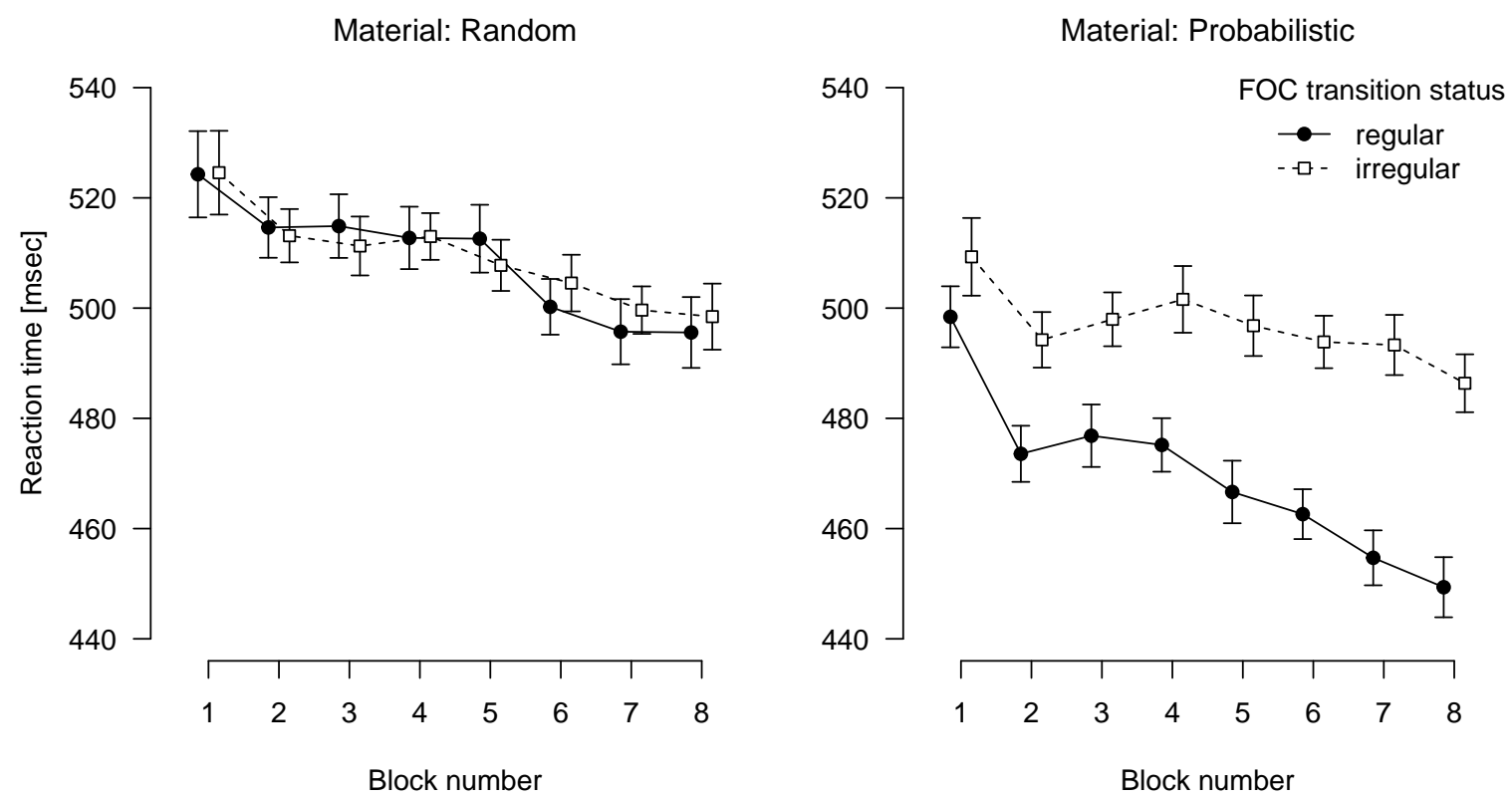

Figure 6. RTs during acquisition phase of Experiment 2, split by material and FOC transition status. Error bars represent $95 \%$ within-subjects confidence intervals.

status, $F(5.93,356.02)=15.83, M S E=194.03, p<.001, \hat{\eta}_{G}^{2}=.007$, showing that the RT difference between regular and irregular transitions increased over blocks, indicating learning of the regularities inherent in the probabilistic material.

Error rates. Figure 7 shows error rates during acquisition. We conducted a 2 (Material: Random vs. Probabilistic) $\times 8$ (Block number $) \times 2($ FOC transition status: regular vs. irregular) ANOVA that revealed a main effect of block number, $F(5.83,693.83)=6.06$, $M S E=11.83, p<.001, \hat{\eta}_{G}^{2}=.016$, indicating that error rates increased over blocks, and a main effect of $F O C$ transition status, $F(1,119)=38.19, M S E=13.49, p<.001, \hat{\eta}_{G}^{2}=.019$, indicating that error rates were higher for nonregular transitions. The interaction of material and FOC transition status was also significant, $F(1,119)=27.61, M S E=13.49, p<.001$, $\hat{\eta}_{G}^{2}=.014$, reflecting the finding that the effect of the latter factor was limited to the probabilistic material. The three-way interaction of material, block number, and FOC transition status was however not significant, $F(6.55,778.97)=1.84, M S E=7.94, p=.082$, $\hat{\eta}_{G}^{2}=.004$.

To disentangle these interactions, we analyzed both material groups separately. As for RT, an ANOVA for the random material group revealed only a main effect of block number, $F(4.94,291.45)=2.50, M S E=16.03, p=.031, \hat{\eta}_{G}^{2}=.013\left(\right.$ all other $\left.F_{\mathrm{s}}<1\right)$. The probabilistic material group showed a main effect of block number $F(5.73,343.65)=$ 4.63, $M S E=10.29, p<.001, \hat{\eta}_{G}^{2}=.022$, and a main effect of FOC transition status, $F(1,60)=62.50, M S E=14.23, p<.001, \hat{\eta}_{G}^{2}=.070$. Importantly, the interaction of block number and FOC transition status was significant, $F(5.9,353.81)=3.23, M S E=7.85$, $p=.004, \hat{\eta}_{G}^{2}=.012$, indicating that the difference in error rates between regular and irregular transitions increased across blocks, consistent with the learning effect obtained for reaction times. 

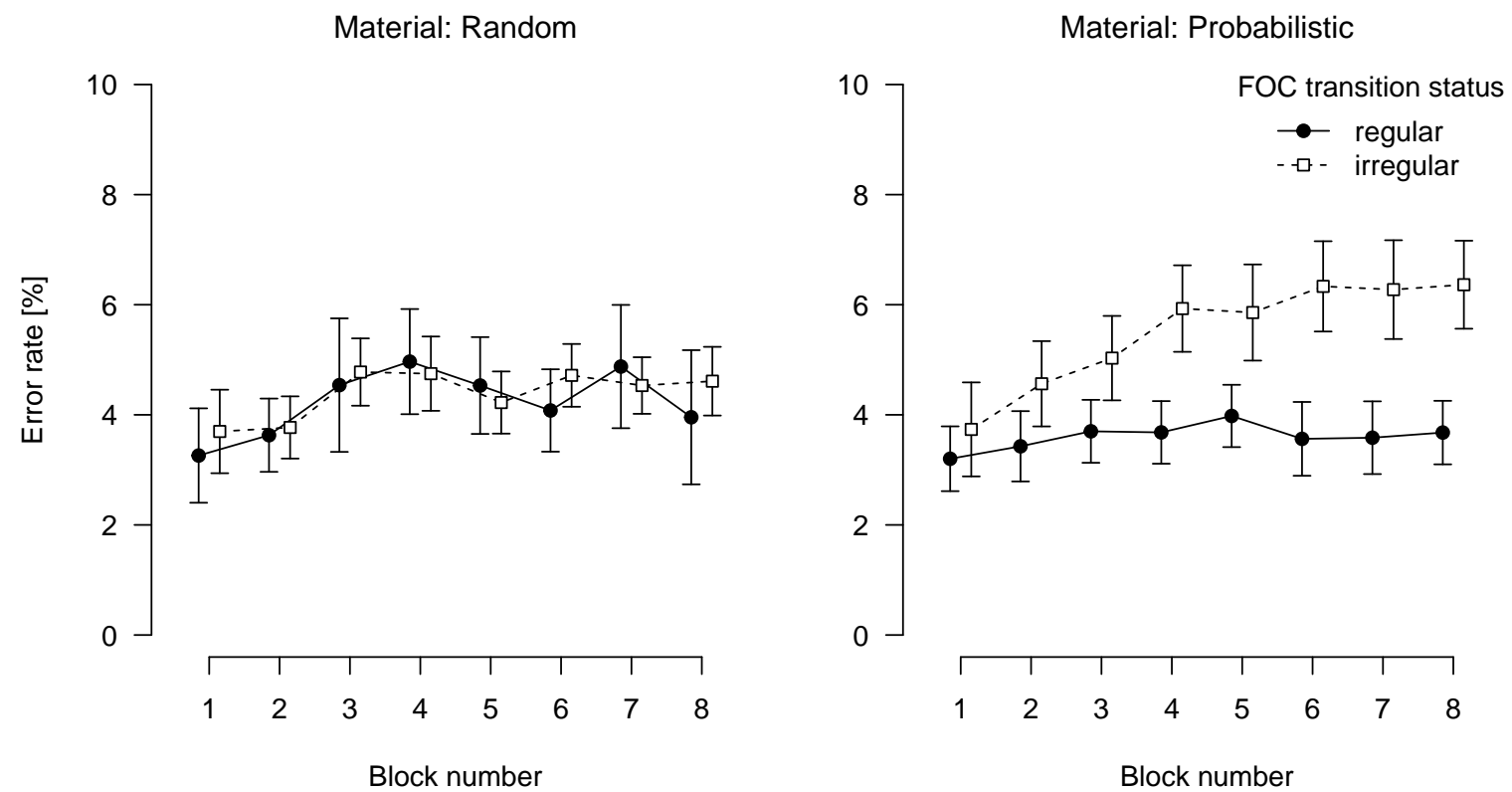

Figure 7. Error rates during acquisition phase of Experiment 2, split by material and FOC transition status. Error bars represent $95 \%$ within-subjects confidence intervals.

Generation task. In a second step, we investigated how learned knowledge was expressed in the generation task. We analyzed generation performance by fitting two hierarchical models, $\mathcal{M}_{1}$ and $\mathcal{M}_{2}$. $\mathcal{M}_{1}$ allows the automatic and controlled processes to vary between inclusion and exclusion, but it assumes that participants acquired only implicit knowledge during the SRTT, and that revealing explicit knowledge after the SRTT did not affect implicit knowledge. $\mathcal{M}_{2}$ is a hierarchical extension of the classical PD model that enforces the invariance assumption. We computed model fit statistics to test whether each model could account for the means, $T_{A 1}$, and covariances, $T_{B 1}$, of the observed frequencies. We compared both models using the DIC statistic that provides a combined assessment of parsimony and goodness of fit and penalizes models for unnecessary complexity. Parameter estimates from model $\mathcal{M}_{1}$ were used to address the invariance assumptions, directly.

The model checks for model $\mathcal{M}_{1}$ were satisfactory,

$$
\begin{gathered}
T_{A 1}^{\text {observed }}=491.06, T_{A 1}^{\text {expected }}=469.94, p=.369, \\
T_{B 1}^{\text {observed }}=9.05, T_{B 1}^{\text {expected }}=6.95, p=.366 .
\end{gathered}
$$

In contrast, the model checks for model $\mathcal{M}_{2}$ revealed significant deviations of the model's predictions from the data,

$$
\begin{gathered}
T_{A 1}^{o b s e r v e d}=1,092.06, T_{A 1}^{\text {expected }}=473.88, p=.002, \\
T_{B 1}^{\text {observed }}=190.05, T_{B 1}^{\text {expected }}=6.93, p<.001 .
\end{gathered}
$$

Model $\mathcal{M}_{1}$ attained a DIC value of $25,293.45$ and clearly outperformed model $\mathcal{M}_{2}$ that attained a DIC value of $25,891.74, \Delta \mathrm{DIC}_{\mathcal{M}_{1}-\mathcal{M}_{2}}=-598.29$. This implies that the 
Automatic processes $A$

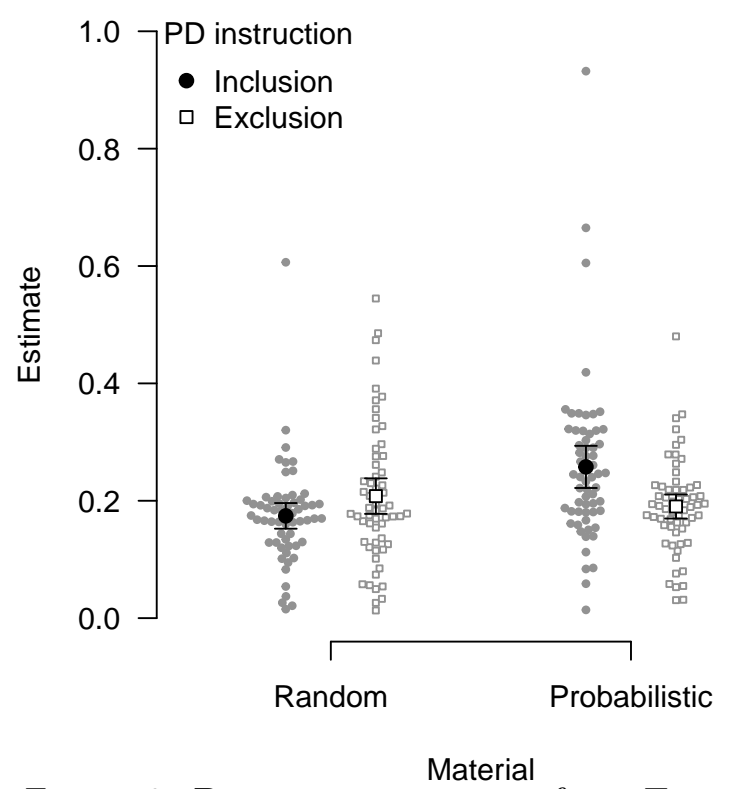

Controlled processes $C$

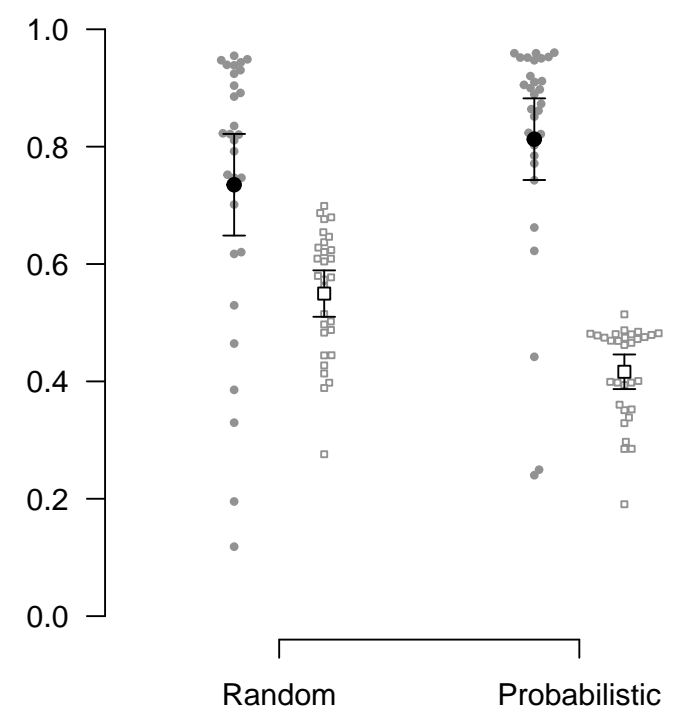

Material

Figure 8. Parameter estimates from Experiment 2. Error bars represent $95 \%$ confidence intervals.

auxiliary assumptions we introduced to $\mathcal{M}_{1}$ were much less problematic than the invariance assumption. Moreover, the standard PD model enforcing the invariance assumption was not able to account for the data.

Figure 8 shows the parameter estimates obtained from model $\mathcal{M}_{1}$. Figure 9 shows that the invariance assumption for the automatic process was violated with $A_{I}>A_{E}, 95 \%$ CI $[.01, .03]$, and a Bayesian $p<.001$ ( $p=.360$ for revealed transitions). The invariance assumption for the controlled process was also violated with $C_{I}>C_{E}, 95 \%$ CI [.08, .54], and a Bayesian $p=.003$.

Robustness checks. Next we assessed whether these findings were sensitive to the assumptions of our models. Despite the fact that the auxiliary assumptions could be upheld in model comparison, and that the incorporating model was well able to account for the data, it may nevertheless still be the case that violations have biased parameter estimates. Specifically, if participants had in fact acquired explicit knowledge about nonrevealed transitions during learning, they may have used this knowledge to generate more regular transitions under inclusion than exclusion. Because of our assumption that $C=0$ for nonrevealed transitions, this performance difference would have been reflected in greater estimates of implicit knowledge under inclusion than exclusion, and might account for the observed $A_{I}>A_{E}$ pattern.

To assess this possibility, we used the questionnaire data to exclude any transitions that participants reported in their explicit description of the sequence (while keeping the revealed transitions); if the acquired explicit knowledge was indeed the cause of the invariance violation, excluding the transitions for which knowledge was reported should make the violation disappear. To the contrary, excluding all correctly reported transitions $(9.04 \%$ of 

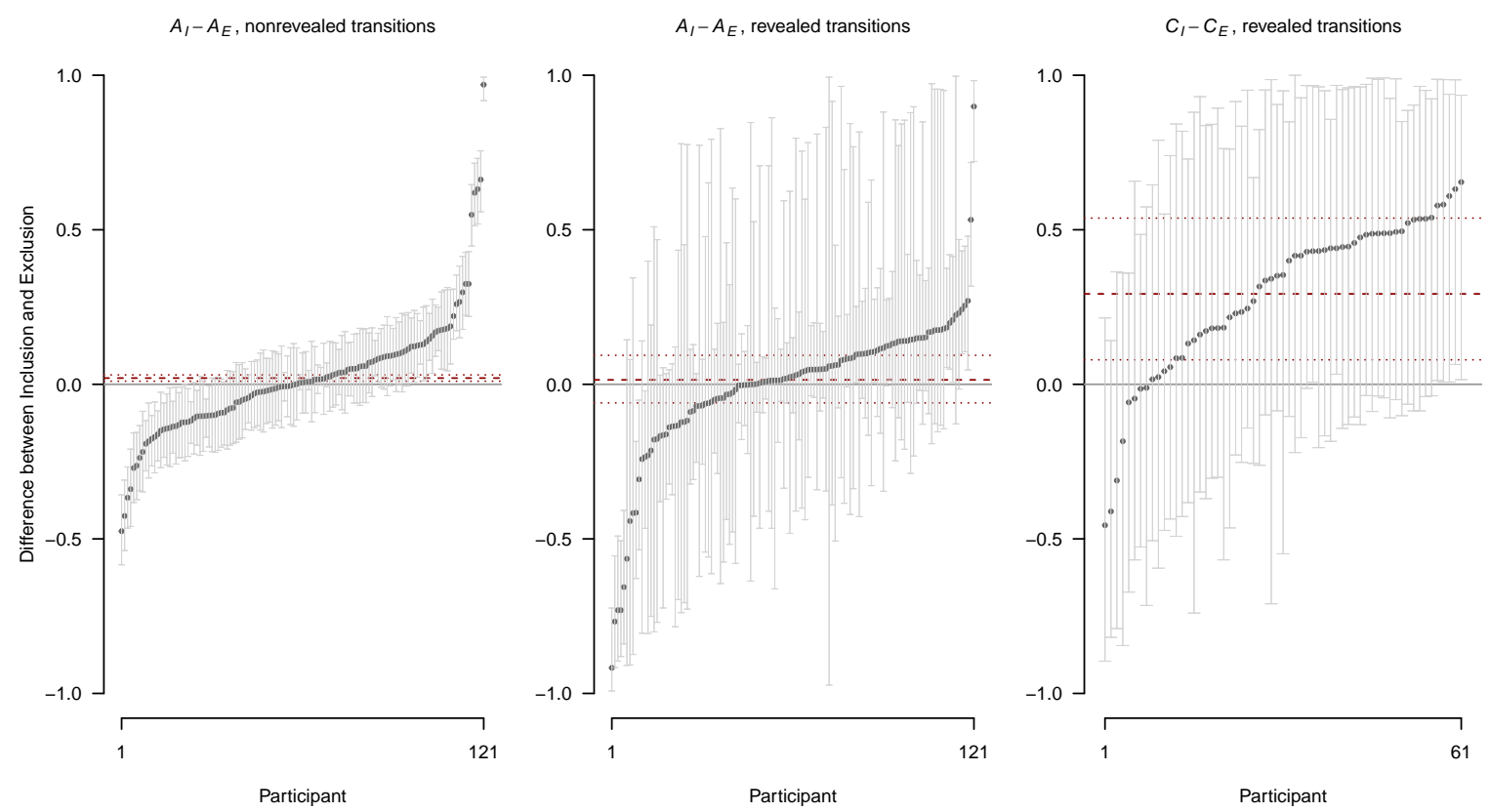

Figure 9. Posterior differences between $A_{I}-A_{E}$ and $C_{I}-C_{E}$ in Experiment 2, plotted for each participant (gray dots) with $95 \%$ credible intervals. Dashed lines represent the posterior means of the differences between mean parameter estimates. Dotted lines represent $95 \%$ credible intervals.

cases) did not affect the pattern of results. ${ }^{13}$

We also tested the invariance assumption using a different model $\mathcal{M}_{1 R}$ that extended $\mathcal{M}_{1}$ by relaxing the assumption that $C=0$ for nonrevealed transitions (see Appendix $\mathrm{C}$ for details). The invariance violation for the controlled process, $C_{I}>C_{E}$, replicated in the absence of the assumption $C=0$, demonstrating its robustness. However, the small invariance violation for the automatic process was no longer evident in $\mathcal{M}_{1 R}$.

\section{Discussion}

Based on the SRTT results, we can conclude that participants acquired sequence knowledge during learning. In addition, explicit knowledge about one of the six transitions had a clear effect on generation performance for that transition.

The extended process-dissociation model $\mathcal{M}_{1}$ revealed a violation of the invariance assumptions for both the controlled process (i.e., $C_{I}>C_{E}$ ) and the automatic process (i.e., $\left.A_{I}>A_{E}\right)$. Model $\mathcal{M}_{1}$ rested on two auxiliary assumptions: It was assumed that controlled processes were not affected by learning material (i.e., no explicit knowledge was acquired from the SRTT), and that automatic processes were not affected by the manipulation of explicit knowledge (i.e., revealing a transition). Both assumptions found support in the current data as they did not harm model fit. Moreover, model comparison by the DIC showed that model

\footnotetext{
${ }^{13}$ Of the reported (nonrevealed) transitions, only approximately $25.47 \%$ were indeed regular transitions. After excluding all reported transitions regardless of whether they reflect correct knowledge or not $(27.55 \%$ of cases), the invariance violation was descriptively unchanged but no longer statistically significant, Bayesian $p=.221$.
} 
$\mathcal{M}_{1}$ was a better account of the data than the standard process-dissociation model $\mathcal{M}_{2}$ that did not impose these assumptions but instead imposed the invariance assumption.

Invariance of the automatic process was significantly violated, but the magnitude of the violation was small, and it disappeared entirely under a relaxed model $\left(\mathcal{M}_{1 R}\right.$; see Appendix C). Given the small magnitude, and its lack of robustness to the modeling assumptions, the invariance violation of $A$ appears to be no serious threat to the validity of the PD at this point.

In contrast, invariance of the controlled process was consistently found to be violated and the violation was large in magnitude: Confirming the speculation that explicit knowledge is not exhaustively used in exclusion, explicit knowledge was used to a greater degree under inclusion than exclusion.

\section{Experiment 3}

The main goal of Experiment 3 was to replicate the previous findings and extend them to second-order conditional (SOC) material.

A secondary goal was to explore whether different amounts of implicit knowledge are acquired with mixed versus pure SOC material. Previous studies of the SRTT using a PD generation task have employed 12-item-sequences of four response locations (e.g., SOC1 = $3-4-2-3-1-2-1-4-3-2-4-1 ;$ SOC2 $=3-4-1-2-4-3-1-4-2-1-3-2$, Destrebecqz \& Cleeremans, 2001; Wilkinson \& Shanks, 2004). Analyzing these sequences more closely, it is evident that they did not only contain second order information (i.e., the last two locations predict the next location), but they also incorporate lower-order information: First, direct repetitions never occur; and reversals occur below chance (i.e., 1/12, whereas chance level would equal $1 / 3$ given that repetitions are prohibited). Second, the last location of a triplet $L_{3}$ is not independent of the first location $L_{1}$ (e.g., for SOC1, $p\left(L_{3}=2 \mid L_{1}=3\right)=2 / 3$ ). In other words, in two out of three cases, the third location of a triplet can be predicted by the first location of a triplet alone. It is plausible that participants are able to learn this lower-order information, and that learning effects may not (only) be based on second-order information (cf., Koch \& Hoffmann, 2000; Reed \& Johnson, 1994). To investigate this possibility, Experiment 3 implemented two types of probabilistic material: A mixed SOC material that incorporated both second-order and first-order types of information, and another pure $S O C$ material that only followed a second-order regularity.

\section{Method}

Design. The study realized a 3 (Material: random, mixed SOC, pure SOC) $\times 2$ (Explicit knowledge: no transition revealed vs. two transitions revealed $) \times 2(P D$ instruction: inclusion vs. exclusion) $\times 2$ (Block order: inclusion first vs. exclusion first) design with repeated measures on the $P D$ instruction factor.

Participants. One hundred and seventy-nine participants (120 women) aged between 18 and 58 years $(M=22.8$ years) completed the study. Most were undergraduates from Heinrich-Heine-Universität Düsseldorf. Data from 8 participants were excluded from generation task analyses because they had received erroneous exclusion instructions. Participants were randomly assigned to experimental conditions. They received either course credit or 3.50 Euro for their participation. 
Materials. We implemented three different types of material:

- A random sequence was randomly generated for each participant anew by drawing with replacement from a uniform distribution of six response locations.

- A mixed $S O C$ sequence incorporated two types of information: First, the third location of a triplet was conditional upon the first two locations. Second, within such regular triplets, given a fixed first-position location, there was one highly probable thirdposition location and two somewhat less probable third-position locations; the other three response locations never occurred for this first-position location.

- A pure $S O C$ sequence followed only the second-order regularity.

In both probabilistic materials (mixed and pure SOC), $87.5 \%$ of trials adhered to the second-order regularity, which was individually and randomly selected for each participant anew. In all conditions, the material adhered to the following (additional) restrictions: (1) there were no direct repetitions of response locations, and (2) there were no response location reversals (i.e., 1-2-1). To compute the dependent variable in the generation task (i.e., the number of rule-adhering triplets), for both probabilistic groups, we used the second-order sequence that was used to generate each participant's materials. For the random group, there is no "regular" sequence and we again computed an individual criterion sequence for each participant. For convenience, we did not generate all possible second-order sequences for these participants (as we did for first-order materials in Experiment 1), but chose to use individual criterion sequences that were randomly generated similar to the pure $S O C$ material.

Procedure. The experimental procedure closely followed that of Experiment 1: In the acquisition task, participants performed a SRTT consisting of eight blocks with 180 trials each (for a total of 1,440 responses). The response-stimulus interval (RSI) was 0 ms. Following the SRTT phase, participants were told that stimulus locations during the SRTT followed some underlying sequential structure. They were then asked to try to generate a short sequence of thirty locations that followed this structure.

The generation task followed, with inclusion versus exclusion block order counterbalanced. We fixed the number of generation-practice blocks that preceded both inclusion and exclusion task: Prior to the inclusion task, three practice blocks involved inclusion instructions; prior to the exclusion task, the first and second practice block involved inclusion instructions, and the third involved exclusion instructions. Before working on practice blocks, two transitions were revealed to one half of the participants.

Upon completing the computerized task, participants were asked to complete a questionnaire containing the following items: (1) "Did you notice anything special working on the task? Please mention anything that comes to your mind.", (2) "One of the tasks mentioned a sequence in which the squares lit up during the first part of the study. In one of the experimental conditions, the squares did indeed follow a specific sequence. Do you think you were in this condition or not?", (3) "How confident are you (in \%)?", (4) "Can you describe the sequence in detail?". Subsequently, participants were asked to indicate, for ten first-order transitions, the next three keys in the sequence on a printed keyboard layout. The first-order transitions were individually selected for each participant so that each participant had the chance to express full explicit knowledge about the second-order regularity. 

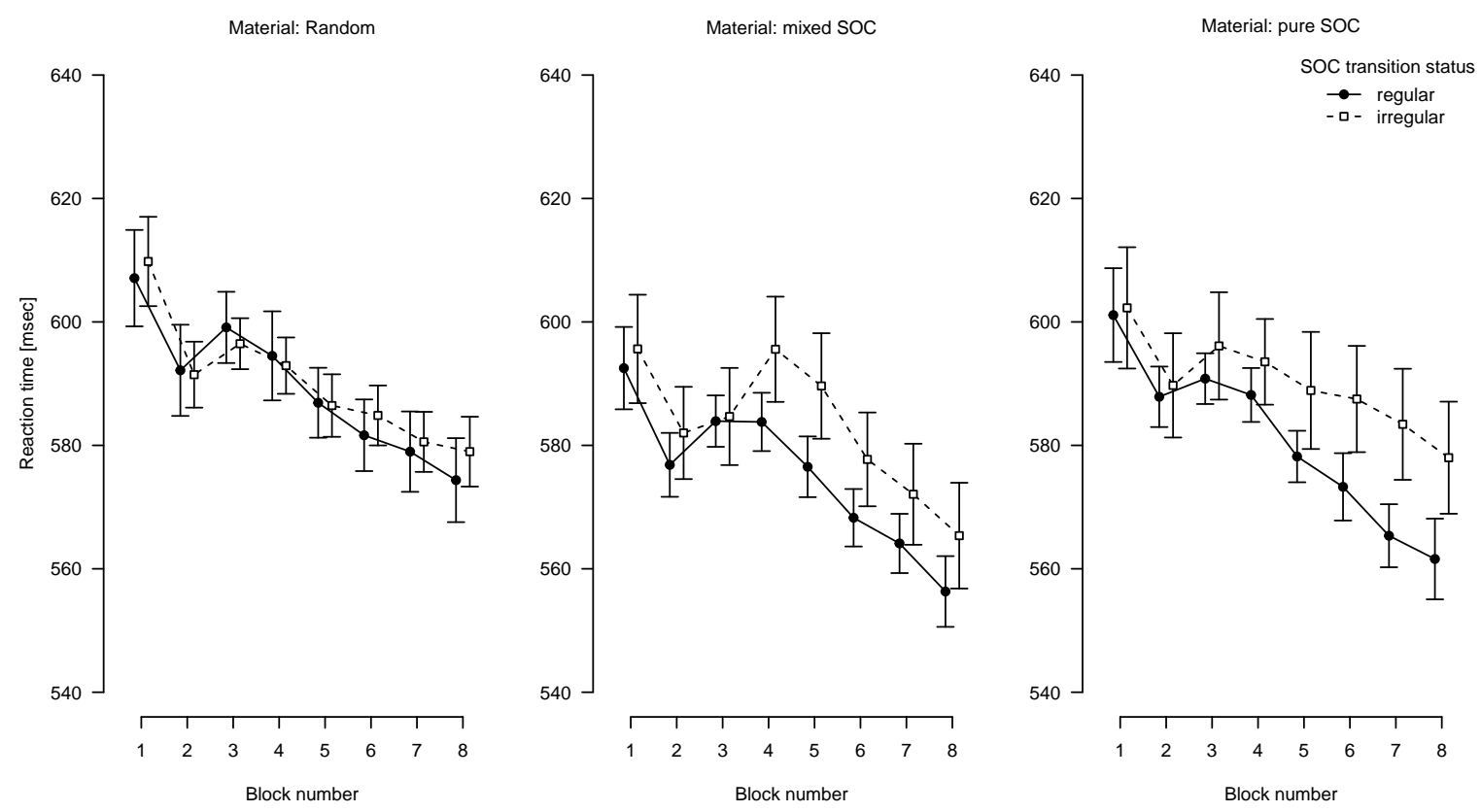

Figure 10. RTs during acquisition phase of Experiment 3, split by material and SOC transition status. Error bars represent $95 \%$ within-subjects confidence intervals.

Data analysis. For analyses of reaction times during the acquisition task, we excluded the first two trials of each block because the first two locations cannot be predicted, as well as error trials, trials succeeding an error, reactions faster than $50 \mathrm{~ms}$ and slower than 1,000 ms. For analyses of error rates during the acquisition task, we excluded the first two trials of each block.

Generation task analyses were conducted with the first two trials of a block as well as any response repetitions and reversals excluded. Model-based analyses were conducted with models $\mathcal{M}_{1}$ and $\mathcal{M}_{2}$ analogous to those used in Experiment 2 (see Appendix D for details).

\section{Results}

We first analyzed reaction times and error rates during the SRTT to determine whether sequence knowledge had been acquired during the task. Next, we analyzed generation task performance using hierarchical PD models (descriptive statistics and ordinal-PD analyses are reported in Appendices A and B).

Acquisition task. If participants acquired sequence knowledge from probabilistic materials, we expect a performance advantage for regular over irregular transitions, reflected in reduced $\mathrm{RT}$ and/or error rate. If this advantage is due to learning, it is expected to increase over SRTT blocks. If participants are able to learn lower-order information that is only present in mixed $S O C$ material, the advantage is expected to be greater in mixed SOC material compared to pure $S O C$. If participants are able to learn second-order information, a performance advantage is to be expected not only in mixed $S O C$ but also in pure $S O C$ material.

Reaction times. Figure 10 shows reaction times during acquisition. We conducted a 3 (Material: random vs. pure SOC vs. mixed SOC) $\times 2$ (Transition status: regular 
vs. irregular SOC $) \times 8$ (Block number $)$ ANOVA with repeated measures on the last two factors that revealed a main effect of block number, $F(4.46,780.51)=41.53, M S E=1,515.93$, $p<.001, \hat{\eta}_{G}^{2}=.020$, reflecting decreasing RT over blocks; a main effect of transition status, $F(1,175)=40.02, M S E=582.10, p<.001, \hat{\eta}_{G}^{2}=.002$, reflecting an RT advantage for regular transitions; and an interaction of block number and transition status, $F(6.39,1118.42)=2.81$, $M S E=439.60, p=.009, \hat{\eta}_{G}^{2}=.001$, reflecting the finding that the RT advantage for regular transitions increased over block (i.e., the sequence learning effect). We also found an interaction of material and transition status, $F(2,175)=7.40, M S E=582.10, p=.001$, $\hat{\eta}_{G}^{2}=.001$, reflecting the finding that the effect of transition status was absent in the random material group, $F(1,58)=0.44, M S E=380.19, p=.510, \hat{\eta}_{G}^{2}=.000$; trivially, no sequence knowledge was learned from random material.

The three-way interaction was not significant, $F(12.78,1118.42)=0.92, M S E=439.60$, $p=.535, \hat{\eta}_{G}^{2}=.000$, suggesting that the sequence-learning effect did not differ across material groups. We conducted separate analyses to probe for sequence-learning effects in each material condition. Analyzing only the random material group revealed only a main effect of block number, $F(3.82,221.55)=15.74, M S E=1,484.04, p<.001, \hat{\eta}_{G}^{2}=.020$ (all other $p \mathrm{~s}>.05$ ). In the pure $S O C$ group, in contrast, a main effect of block number, $F(3.96,229.51)=12.04, M S E=2,038.65, p<.001, \hat{\eta}_{G}^{2}=.019$, was accompanied by a main effect of transition status, $F(1,58)=28.48, M S E=637.73, p<.001, \hat{\eta}_{G}^{2}=.004$, and an interaction of both factors, $F(6.03,349.61)=2.47, M S E=530.13, p=.023, \hat{\eta}_{G}^{2}=.002$, reflecting a sequence learning effect on RT.

In the mixed SOC group, we obtained only main effects of block number, $F(4.91,289.7)=15.95, M S E=1,334.22, p<.001, \hat{\eta}_{G}^{2}=.024$, and of transition status, $F(1,59)=18.83, M S E=725.90, p<.001, \hat{\eta}_{G}^{2}=.003$, but the interaction of block number and transition status was not significant, $F(5.74,338.77)=1.15, M S E=571.40$, $p=.331, \hat{\eta}_{G}^{2}=.001$. This is despite the fact that the effect of transition status is also likely to be a result of sequence learning, and it is of similar magnitude to that obtained in the pure SOC group. The notion that both learning effects are similar was also supported by a joint analysis of the pure SOC and mixed SOC groups: The two-way interaction between block number and transition status was significant, $F(6.14,718.32)=2.75, M S E=527.50$, $p=.011, \hat{\eta}_{G}^{2}=.001$, but the three-way-interaction of material, block number, and transition status was not significant, $F(6.14,718.32)=0.87, M S E=527.50, p=.521, \hat{\eta}_{G}^{2}=.000$. Taken together, we interpret these findings to show that the learning effect in the mixed SOC group was comparable to that observed in the pure SOC group but too small to reach significance in a separate analysis.

Error rates. Figure 11 shows error rates during acquisition. We conducted a 3 (Material: Random vs. mixed SOC vs. pure SOC $) \times 8$ (Block number $) \times 2($ SOC transition status: regular vs. irregular) ANOVA with repeated measures on the last two factors that revealed a main effect of block number, $F(3.66,644.87)=3.78, M S E=39.10, p=.006$, $\hat{\eta}_{G}^{2}=.008$, reflecting increasing error rates over blocks, and a main effect of transition status, $F(1,176)=16.14, M S E=9.08, p<.001, \hat{\eta}_{G}^{2}=.002$, reflecting an accuracy advantage for regular transitions. The interaction of material and transition status was not significant, $F(2,176)=2.66, M S E=9.08, p=.073, \hat{\eta}_{G}^{2}=.001$,

Separate analyses yielded no significant effects in the random material group (all $p \mathrm{~s}>$ .05). Importantly, an effect of transition status was clearly absent from the random material 

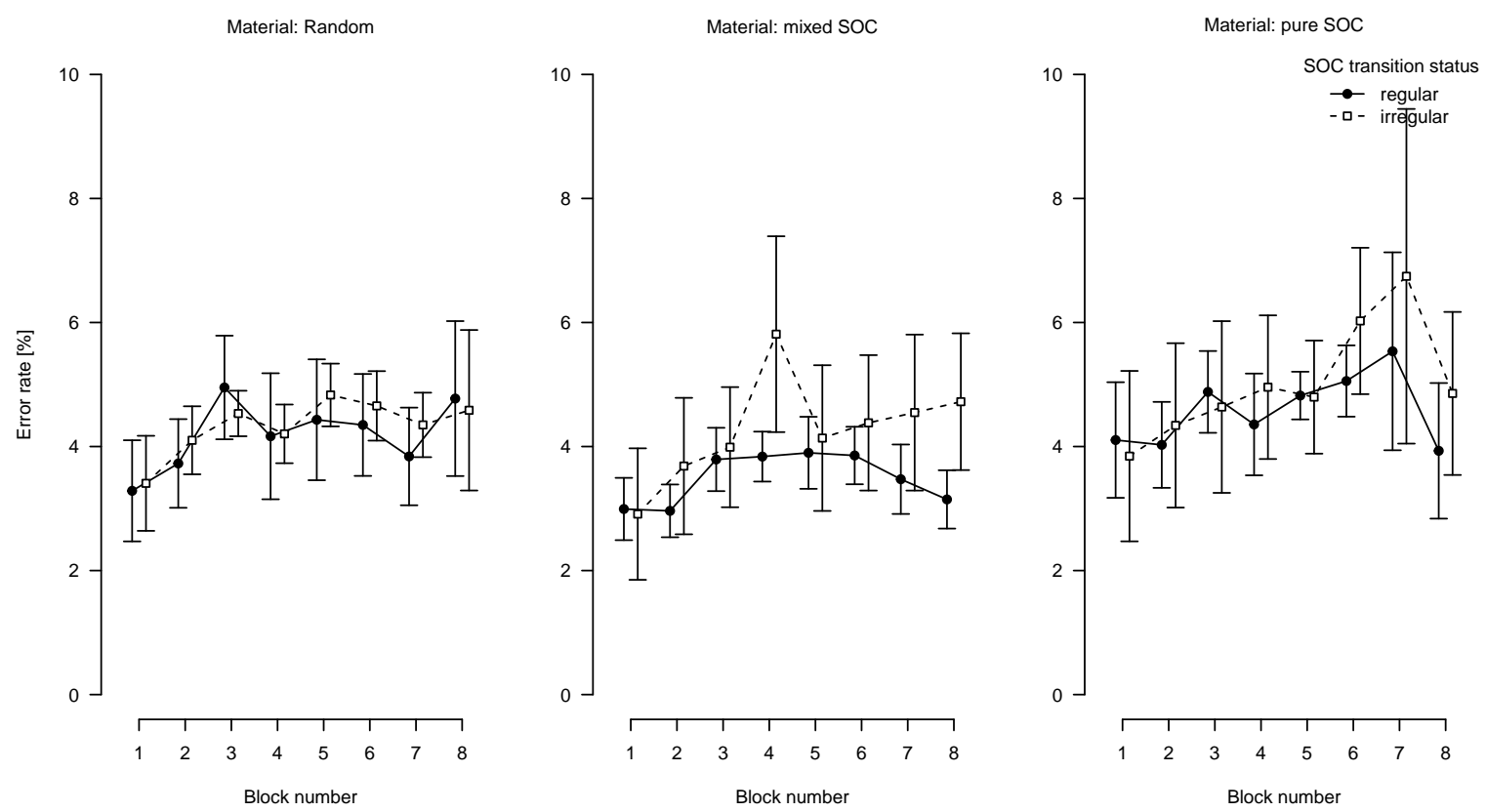

Figure 11. Error rates during acquisition phase of Experiment 3, split by material and SOC transition status. Error bars represent $95 \%$ within-subjects confidence intervals.

group, $F(1,58)=0.62, M S E=7.68, p=.433, \hat{\eta}_{G}^{2}=.000$. In the mixed SOC group, a main effect of block number was found, $F(5.66,334.01)=2.96, M S E=15.46, p=.009, \hat{\eta}_{G}^{2}=.017$, along with a main effect of transition status, $F(1,59)=12.88, M S E=11.29, p=.001$, $\hat{\eta}_{G}^{2}=.009$, reflecting higher error rates for irregular than for regular transitions. Finally, in the pure $S O C$ group, block number did not affect error rates, $F(1.87,110.6)=1.72$, $M S E=133.60, p=.185, \hat{\eta}_{G}^{2}=.011$; but a main effect of transition status was also found, $F(1,59)=5.55, M S E=8.24, p=.022, \hat{\eta}_{G}^{2}=.001$, reflecting higher error rates for irregular than regular transitions.

Taken together, error rates mirror RTs in that they also reflect a performance advantage for regular transitions in the mixed and pure SOC groups that was not evident in the random control group. Deviating from the RT result pattern, this advantage did not reliably increase across blocks.

Generation task. We analyzed generation performance by fitting the two hierarchical models $\mathcal{M}_{1}$ and $\mathcal{M}_{2}$ that we introduced above to the data from Experiment 3 . For both models, we computed model fit statistics to assess whether each model could account for the data; we then compared both models using the DIC. Parameter estimates from model $\mathcal{M}_{1}$ were then used to address the invariance assumptions directly.

The model checks for model $\mathcal{M}_{1}$ were satisfactory,

$$
\begin{gathered}
T_{A 1}^{\text {observed }}=692.77, T_{A 1}^{\text {expected }}=653.45, p=.291, \\
T_{B 1}^{\text {observed }}=8.44, T_{B 1}^{\text {expected }}=6.04, p=.292 .
\end{gathered}
$$

In contrast, the model checks for model $\mathcal{M}_{2}$ revealed significant deviations of the model's 
Automatic processes $A$

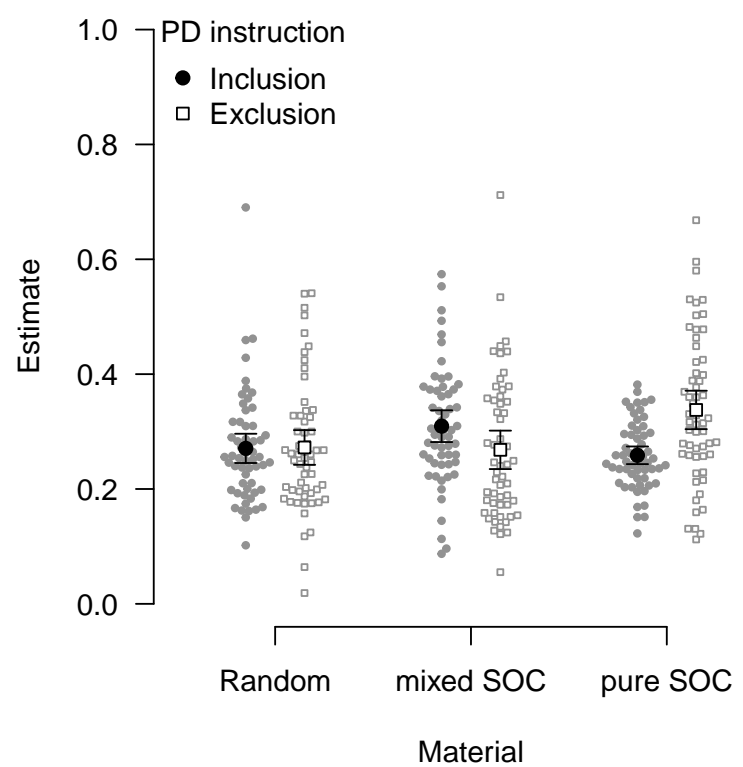

Controlled processes $C$

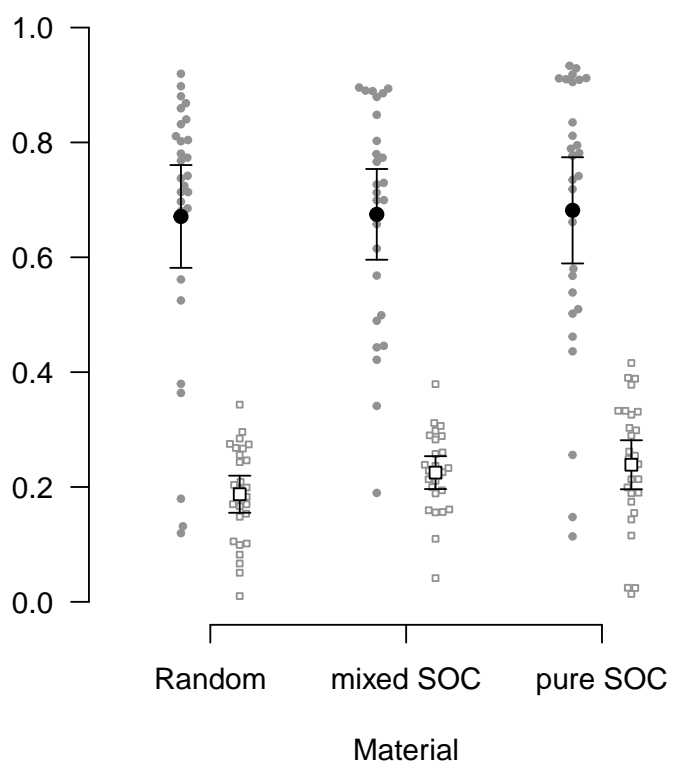

Figure 12. Parameter estimates from Experiment 3. Error bars represent 95\% confidence intervals.

predictions from the data,

$$
\begin{gathered}
T_{A 1}^{\text {observed }}=1,077.52, T_{A 1}^{\text {expected }}=652.79, p=.003, \\
T_{B 1}^{\text {observed }}=49.97, T_{B 1}^{\text {expected }}=6.06, p<.001 .
\end{gathered}
$$

Model $\mathcal{M}_{1}$ attained a DIC value of $38,907.43$ and outperformed model $\mathcal{M}_{2}$ that attained a DIC value of $39,210.66, \Delta \mathrm{DIC}_{\mathcal{M}_{1}-\mathcal{M}_{2}}=-303.23$. This implies that our auxiliary assumptions were less problematic than the invariance assumption. Moreover, the standard PD model enforcing the invariance assumption was not able to account for the data.

Figure 12 shows the parameter estimates obtained from model $\mathcal{M}_{1}$. Figure 13 shows that the invariance assumption for controlled processes was again violated with $C_{I}>C_{E}$, $95 \%$ CI $[.27, .63]$, Bayesian $p<.001$. The invariance violation was also obtained with model $\mathcal{M}_{1 R}$, showing that it is robust to the specific modeling assumptions (see Appendix $\mathrm{C}$ ). In contrast to the results of Experiment 2, the invariance assumption for automatic processes was not violated but could be upheld, $95 \%$ CI $[-.01, .01]$, Bayesian $p=.638$ for non-revealed transitions and $95 \%$ CI $[-.10, .05], p=.763$ for revealed transitions.

\section{Discussion}

Based on the SRTT results, we can conclude that participants acquired some (albeit weak) sequence knowledge during learning. In addition, generation performance was clearly affected by instructed explicit knowledge, as revealed by the clearly above-zero estimates of the $C$ parameters for revealed transitions. 

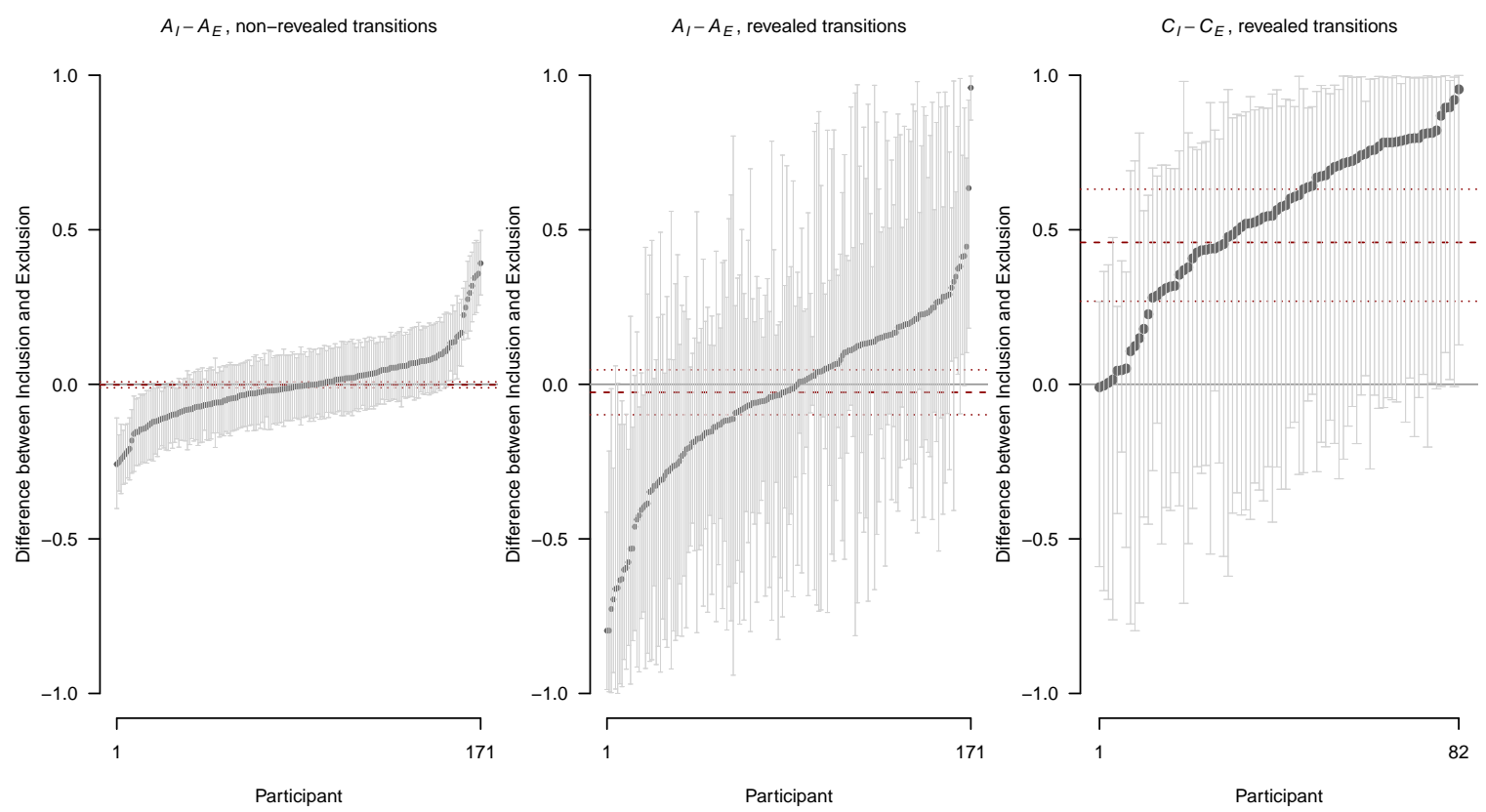

Figure 13. Posterior differences $A_{I}-A_{E}$ and $C_{I}-C_{E}$ in Experiment 3, plotted for each participant (gray dots) with $95 \%$ credible intervals. Dashed lines represent the posterior means of the differences between mean parameter estimates. Dotted lines represent $95 \%$ credible intervals.

An extended process-dissociation model $\mathcal{M}_{1}$ revealed a violation of the invariance assumption for controlled processes with $C_{I}>C_{E}$. The invariance assumption for automatic processes could be upheld. Model $\mathcal{M}_{1}$ rested on two auxiliary assumptions: It was assumed that controlled processes were not affected by learning material, and that automatic processes were not affected by the manipulation of explicit knowledge. Both assumptions found support in the current data as they did not harm model fit. Moreover, model selection strongly favored model $\mathcal{M}_{1}$ over a standard process-dissociation model $\mathcal{M}_{2}$ that did not impose these assumptions.

Regarding our secondary goal to explore whether different amounts of sequence knowledge are acquired from mixed versus pure second-order conditional material, we did not find evidence for a difference between these two types of material in the SRTT. This may well be due to the overall low levels of acquired sequence knowledge in the present study. Clearly, the present data are not strong enough to rule out such differences; this question requires further study.

\section{General Discussion}

\section{Summary of main findings}

The process-dissociation approach as applied to sequence learning assumes either (1) that automatic processes monotonically increase both inclusion and exclusion performance, while controlled processes increase inclusion but decrease exclusion performance (if the ordinal approach is used), or (2) that the controlled and automatic process are invariant 
under inclusion and exclusion instructions (if the parametric model is used).

In three sequence-learning experiments, we tested whether the monotonicity and invariance assumptions hold in the generation task. The results show a consistent pattern.

Monotonicity assumption. Increases in explicit knowledge across conditions consistently increased overall inclusion performance, but were insufficient to reliably decrease overall exclusion performance. Participants were largely unable to use their explicit knowledge to suppress the proportion of regular transitions generated in the exclusion task to levels below baseline. Below-baseline generation levels for revealed transitions were robustly found only for material with a first-order regularity, and only in participants who had explicit knowledge about (at least) two transitions and engaged in generation-task practice specific to a given to-be-excluded transition (Exp. 1, Transfer condition). In these participants, there was even some evidence that below-chance exclusion performance transferred to non-practiced explicit knowledge. However, transition-specific practice was (necessary but) not sufficient for successful exclusion: Whereas participants without such practice (i.e., the No-Practice and Unspecific-Practice conditions of Exp.1) failed to reach below-chance levels, participants with practice also failed to attain below-chance levels under exclusion instructions if they worked on the inclusion task first (i.e., Exp. 1, Practice condition). Taken together, these results confirm Wilkinson and Shanks's (2004) speculation that inclusion and exclusion strategies may differ and that explicit knowledge is not exhaustively expressed in the generation task's exclusion condition, to the effect that increasing explicit knowledge does not result in decreased generation of regular transitions under exclusion.

Invariance of the controlled process. The finding that explicit knowledge was less likely to affect exclusion performance also suggests a violation of invariance. Experiments 2 and 3 showed that, indeed, the invariance assumption for explicit knowledge was consistently violated, in first-order as well as second-order material, and despite extensive opportunity for practice. In all cases, explicit knowledge was expressed to a greater degree under inclusion than under exclusion instructions: Participants succeeded in generating the revealed transition under inclusion conditions, but failed to consistently refrain from generating that transition under exclusion conditions; specifically, under exclusion conditions, participants typically generated the revealed transition at chance levels, instead of suppressing its generation altogether as instructed.

\section{Limitations and open questions}

Before turning to the implications of the present findings, we discuss potential limitations and identify open questions.

The invariance violation of the automatic process may reflect learned explicit knowledge. In Experiment 2 that used first-order conditional material we found evidence suggesting a violation of the invariance assumption for implicit knowledge; no such evidence was however found for the second-order conditional material used in Experiment 3. If interpreted in a standard PD framework, the inclusion-exclusion performance difference resulting from this violation may lead to erroneous conclusions about the presence of explicit knowledge (if such knowledge is indeed absent), or to overestimation of the contribution of explicit knowledge. We believe these findings of an inclusion-exclusion difference in estimates of the automatic parameter should be interpreted with some caution, for at least three reasons. First, the finding was inconsistent across studies, and there are multiple possible 
causes of this inconsistency: The lack of a violation in Experiment 3 may be due to specific properties of the material, or it may be due to the fact that sequence knowledge levels in that study were too low for differences in its expression to be measurable.

Second, the violation was relatively small (i.e., the $A_{I}-A_{E}$ difference ranged between .01 and .03 in Exp.2; and between .00 and .03 in Exp.1, see Appendix C). In the absence of controlled influences, this would be equivalent to a difference between inclusion and exclusion performance of approximately 2 percentage points - an effect barely noticeable under typical conditions.

Third, it is unclear whether the observed invariance violation of parameter $A$ reflects implicit knowledge at all. Note that the parameter for the automatic process captures the sum of all non-controlled influences on generation performance. In particular, it might reflect guessing strategies, and these may differ under inclusion versus exclusion conditions (Stahl et al., 2015). In other words, the above effect may reflect a violation of invariance of guessing or response strategies instead of a violation of invariance of the automatic expression of implicit knowledge. Taken together, we interpret the finding as too weak to conclude that the invariance assumption is violated also for the automatic process.

Instead of being due to guessing, the inclusion-exclusion difference in estimates of the automatic parameter may be due to explicit knowledge acquired during learning. Such an effect, if present at all, is likely to be small given that (1) the material was probabilistic and therefore difficult to learn explicitly; (2) the model incorporating the assumption that no learned explicit knowledge was learned fitted the data well; and (3) the results were unchanged when we excluded the data from transitions that participants (correctly) reproduced during debriefing. However, we cannot exclude the possibility that small amounts of explicit knowledge, obtained during the SRTT phase, may have distorted our model's parameter estimates. This interpretation could also account for the lack of such an effect in Experiment 3 given that explicit knowledge was less likely to be learned from the more complex second-order conditional material used in that study. If this were true, then any differences between inclusion and exclusion that were attributed by the model to an invariance violation of the implicit process may in fact have been a consequence of residual explicit knowledge that was not reflected in our debriefing questionnaire (perhaps due to participants' conservative reporting criteria).

To further address this possibility, we conducted additional model analyses for Experiments 2 and 3 (reported in Appendix C) that aimed at estimating the amount of this residual explicit knowledge; we still found a violation of invariance for the automatic process, but of different direction - a finding that we consider to be an artifact of the auxiliary modeling assumptions. This limitation is another reason for caution in interpreting the above finding as evidence for a violation of invariance of the automatic process. Note that it does not limit the interpretation of our main finding of the invariance violation of the controlled process, which was robust against changes in auxiliary assumptions.

The evidence for sequence learning was weak for SOC material in Experi-

ment 3. As expected, second-order conditional material (Experiment 3) was more difficult to learn than first-order conditional material (Experiments 1 \& 2). This was reflected here in the finding that (despite a 20\% increase in learning trials) there was only weak evidence for sequence learning in Experiment 3. Specifically, responses to regular transitions were clearly faster and more accurate for both variants of the SOC materials, but the interaction 
between regularity and training block, which is critical for unambiguously interpreting a performance advantage for regular transitions as an effect of learning, was not significant. Clearly, an even larger amount of SRTT training should be realized in future studies using SOC materials. Yet, it is unlikely that the observed RT advantage for regular transitions has any other causes than learning, given that it was absent from the random condition, and that the effect could not be attributed to properties of specific transitions because regularity of a transition was randomized for each participant anew. Nevertheless, because evidence for (implicit) sequence learning was not beyond doubt, it is not warranted to interpret the modeling results as stringent tests of the invariance assumption for the automatic process.

Explicit knowledge learned via instruction may be qualitatively different from acquired explicit knowledge. The present study manipulated explicit knowledge via instruction. Although it is an established method (e.g., Liefooghe, Wenke, \& De Houwer, 2012) that has yielded important insights in other domains, one might argue that explicit knowledge acquired via instruction is somehow qualitatively different from explicit knowledge acquired during SRTT training, and that therefore the present results do not speak to the question of interest regarding the invariance of the expression of acquired knowledge. We believe our manipulation to be valid for the following reasons. First, the instructed explicit knowledge communicated the same proposition about the sequence that participants would have acquired during SRTT training (i.e., that a specific location was regularly followed by another location). Second, we took precautions to avoid any inconsistency or conflict with learned sequence knowledge: Transitions that were revealed to participants were part of the regular sequence and therefore compatible with acquired (implicit or explicit) sequence knowledge. Third, we allowed participants to integrate instructed and acquired knowledge during the practice blocks before the generation task.

Given that the instructed and acquired propositions are identical, we would argue that qualitative differences between acquired and instructed knowledge are likely to involve nonpropositional forms of knowledge; such non-propositional knowledge is typically considered to be implicit. Indeed, it is likely that strong implicit knowledge is a precondition for acquiring explicit knowledge (Cleeremans \& Jiménez, 2002; Haider \& Frensch, 2009): Instructed and acquired explicit knowledge are therefore likely to differ in the degree to which they are correlated with implicit knowledge. If participants are better able to control acquired than instructed explicit knowledge, this would then be due, paradoxically, to the presence of acquired implicit knowledge. Finally, even if that was the case, note that this would not salvage the PD method because a strong correlation between explicit and implicit knowledge would violate the independence assumption, thereby posing another problem for its validity.

Boundary conditions of the process-dissociation approach may be violated. Jacoby and colleagues (Jacoby, Begg, \& Toth, 1997; Jacoby, Toth, \& Yonelinas, 1993; Toth, Reingold, \& Jacoby, 1994) emphasized the importance of avoiding floor effects when applying the process-dissociation approach. In the present studies, floor effects may be present if participants succeed in avoiding to generating any regular transitions under exclusion instructions. In such cases, parameter estimates of controlled and automatic processes may be biased and might have artifactually produced an invariance violation for controlled processes (i.e., $C_{I}>C_{E}$ ). We would argue this is not the case in our data for the following reasons: First, generation performance in the present studies fails to show evidence for floor effects (i.e., both low overall levels as well as reduced variability): Regarding overall levels, 
mean performance in most conditions deviated no more than $\pm 1 S D$ from chance baseline (i.e., 20\% in Experiments 1 and 2, and 25\% in Exp.3). Regarding variability, the cells with the lowest overall performance (i.e., with the greatest risk of floor effects) showed variability comparable to that exhibited by the other conditions. While we found below-chance exclusion performance for revealed transitions in some conditions of our experiments (i.e., successful exclusion of the revealed transitions), the invariance violation for controlled processes was replicated not only in these conditions, but across all conditions that involved non-zero explicit knowledge. Importantly, it also replicated in Experiment 3 that realized a higher baseline level of 25\%: In that study, revealed transitions were generated at rates of $25-30 \%$ under exclusion conditions, rates that are clearly unconspicuous of reflecting floor effects.

Second, while Jacoby and colleagues warned about floor effects, they also described the mechanism by which zero counts pose a threat to the validity of the estimation procedure, and proposed means to deal with this problem: If individual participants' data are analyzed separately, floor effects might be accompanied by inflated levels of zero counts in the exclusion condition (i.e., perfect exclusion) for some participants. The original PD equations would then lead to an estimate of $A=0$ for this participant; therefore, averaging over individual $A$ parameters would lead to an underestimation of the automatic parameter. As a means to circumvent this estimation problem, Toth et al. (1994) proposed complete pooling (i.e., aggregate analysis) of data. In our studies, we followed and extended this recommendation by utilizing a Bayesian hierarchical multinomial model for estimating parameters; while allowing for individual differences between parameter estimates, a participant's parameter estimate is not only affected by the data that are directly linked to this estimate, but also by the higher-level distribution for this parameter; the influence of outlier values (such as zero counts provided by some individuals) on the parameter estimates is thus minimized. Another type of estimation bias may arise if our data contained otherwise inflated levels of zero counts; this would suggest higher levels of control ability and lead to an overestimation of $C$ parameters for exclusion conditions. Such inflated levels of zero counts would therefore work against our main invariance-violation finding that explicit knowledge remains underutilized under exclusion conditions (i.e., $C_{I}>C_{E}$ ).

Finally, our conclusion that explicit knowledge remains underutilized under exclusion is not only based on analyses of the parametric PD model (which are susceptible to biased parameter estimates due to floor effects) but was consistently corroborated by the results from ordinal-PD analyses that did not depend on estimates based on the parametric PD equations: Across all three studies, we consistently found a violation of the monotonicity assumption in the sense that explicit knowledge does not reliably decrease the proportion of regular transitions in exclusion conditions (see Appendices A and B).

\section{Implications}

We will first discuss implications for the PD approach before we suggest ways to improve measurement of sequence knowledge using the generation task. We conclude with a few broader implications.

Validity of the PD method. The present findings show that participants fail to exhaustively suppress generating regular transitions under exclusion instructions; this finding has repercussions for both the ordinal- and parametric-PD approaches. 
In the ordinal approach, given a single experimental condition, it is concluded that implicit knowledge is present if exclusion performance is above a (chance or empirical) baseline; and it is concluded that explicit knowledge is present if inclusion performance exceeds exclusion performance. These conclusions depend on the assumption that a monotonically increasing controlled process should lead to a monotonic increase of inclusion performance and at the same time a monotonic decrease of exclusion performance. The present study shows, however, that exclusion performance cannot be assumed to reliably decrease with increasing explicit knowledge. This implies that the assumptions underlying the ordinal-PD approach are violated for the generation task as applied to sequence learning. In addition, we have previously shown that another assumption of ordinal PD, namely that baseline performance is identical in the inclusion and exclusion tasks, is also violated at least in some cases (Stahl et al., 2015). Given that these two fundamental assumptions are violated, the analysis approach adopted in the SRTT literature is also compromised.

The controlled process was found to operate less effectively under exclusion than inclusion instructions; in terms of the parametric PD model, invariance for the controlled process was violated with $C_{I}>C_{E}$. A model that nevertheless incorporates the invariance assumption will likely fail to adequately account for the data, and will yield distorted estimates of the automatic and controlled process. To illustrate, assume that the true values of the parameters are $C_{\text {Inclusion }}=.8, C_{\text {Exclusion }}=.4$, and $A_{\text {Inclusion }}=A_{\text {Exclusion }}=.25$. This yields the following generation proportions of regular transitions $I=.8+(1-.8) * .25=0.85$ and $E=(1-.4) * .25=0.15$. When fitting a traditional PD model enforcing the invariance assumption $C=C_{\text {Inclusion }}=C_{\text {Exclusion }}$ to these data, we get $C=.7$ that lies somewhere between the true values of $C$, and $A=.5$ which is a vast overestimation of the true $A$. Importantly, note that if the true value of $A=.25$ represents chance level, applications of the traditional PD method might lead to the erroneous conclusion that implicit knowledge had been learned even if such knowledge was in fact entirely absent. In addition, if we are interested in the amount of explicit knowledge learned from the SRTT training phase, it might be argued that the higher estimate obtained from the inclusion condition might be a more valid estimate of learned explicit knowledge; the inability to express this knowledge under exclusion may be of secondary interest. By this argument, applying the traditional PD method also yields an underestimation of explicit knowledge.

We therefore recommend against using the PD method unless separate estimates of $C_{\text {Inclusion }}$ and $C_{\text {Exclusion }}$ can be obtained, for example as we have done in the present study. To do so, an extension of the standard design is necessary; for instance, in the present study we implemented two levels of an explicit-knowledge factor across which we equated the $A$ parameters; this allowed us to estimate separate $C$ parameters for inclusion and exclusion. Note that this strategy may not be broadly applicable in typical SRTT studies because of the strong correlation between (acquired) $C$ and $A$; the assumption that the level of implicit knowledge is constant across two different levels of explicit knowledge will be warranted only in special cases such as realized in the present studies (e.g., if explicit knowledge is revealed).

Generation task as a measure of sequence knowledge. The generation task has been proposed as a useful and sensitive measure of implicit knowledge (Jiménez et al., 1996; Perruchet \& Amorim, 1992). Its sensitivity may be called into question by the finding that RT effects obtained during the SRTT were often greater than implicit-knowledge effects in the generation task. In part, this may be attributed to the greater reliability of the RT 
measure, as it relies on aggregation across a larger number of trials than does the generation task. Another possible reason is that the generation task's sensitivity as a measure of implicit knowledge may be lower than previously thought. For instance, previous findings of implicit knowledge using the generation task may have been overestimates of implicit knowledge due to a violation of invariance for the controlled process with $C_{I}>C_{E}$. Note that most studies used much easier-to-learn materials (with four instead of six locations); it is thus plausible that participants acquired more explicit knowledge than they did in our experiments, and that the overestimation bias was more severe in those studies.

Another possible reason for overestimating implicit knowledge is that the regularities in the sequences implemented in previous research were such that the probability of reversals (e.g., 1-2-1) was below chance. Given that participants spontaneously tend to generate reversals at below-chance levels, this implies that they instead generate other regular transitions at slightly above-chance levels even in the absence of any true sequence knowledge (Stahl et al., 2015). As a consequence of this reversal-avoidance bias, implicit knowledge might be overestimated if one uses chance baselines as a reference. This problem has been discussed before (Destrebecqz \& Cleeremans, 2003; Reed \& Johnson, 1994; Shanks \& Johnstone, 1999), and was solved by comparing performance on the training sequence with performance on a transfer sequence containing a similarly low proportion of reversals. This implies, however, that the PD approach does not provide a measure of the absolute level of implicit or explicit knowledge; instead, by relying on a comparison of performance across two sequences, it yields a difference measure that is associated with reduced reliability. In addition, the reversal-avoidance bias may not only mimic implicit knowledge; it may also mimic (or mask) explicit knowledge if it interacted with the inclusion-exclusion instructions, perhaps via different response strategies or criteria adopted under inclusion versus exclusion instructions.

\section{Conclusion and Outlook}

In light of the present findings suggesting limited validity of the PD generation task, what can we conclude about explicit and implicit sequence knowledge from its previous applications? Clearly, the violation of basic assumptions implies that PD results cannot be unambiguously interpreted: Unless we have a better understanding of the processes that drive generation performance, and the degree to which they operate under inclusion versus exclusion instructions, comparisons between inclusion and exclusion performance do not support conclusions about implicit and explicit knowledge. This also implies that a reanalysis of previous findings (which is beyond the scope of the present article) would probably provide limited insight. In this section we therefore take a different approach: We initially accept the conclusions reported in the literature about the contribution of implicit and explicit knowledge at face value; consider the implications of these conclusions about the presence of distortions arising from the invariance violation; and then discuss how the initial conclusion should be corrected in light of these distortions. To recap, the invariance violation results in overestimation of implicit knowledge and underestimation of explicit knowledge. These distortions differentially affect the three patterns of results found in the literature (i.e., evidence for only implicit knowledge, for only explicit knowledge, or both).

The first pattern, evidence for implicit but no explicit knowledge, was found in only two studies (no-RSI condition, Destrebecqz \& Cleeremans, 2001; and Exp.3, 6-blocks condition, 
Q. Fu et al., 2008). In these studies, however, explicit knowledge may nevertheless have been acquired; the observed lack of significant evidence for explicit knowledge may instead reflect the underestimation bias resulting from the invariance violation, perhaps combined with relatively low statistical power (with $N=12$ and $N=24$ in the respective conditions).

Other attempts to replicate this finding were unsuccessful and instead produced the second, opposite, pattern - evidence for explicit but no implicit knowledge (e.g., Wilkinson \& Shanks, 2004). In this case, the evidence for explicit knowledge suggests that the distortions due to the invariance violation apply: Obtaining evidence for explicit knowledge despite the underestimation bias implies that explicit knowledge was likely present. Obtaining no evidence for implicit knowledge despite the likely presence of an overestimation bias supports the absence of implicit knowledge (or, alternatively, it may reflect lack of statistical power).

The third pattern - evidence for both explicit and implicit knowledge - was reported in several studies (e.g., Destrebecqz \& Cleeremans, 2001, 2003; Jiménez, Vaquero, \& Lupiáñez, 2006). The evidence for explicit knowledge suggests that the distortions resulting from the invariance violation may have compromised the results: Again, the evidence for explicit knowledge obtained despite the underestimation bias should probably be assumed to be reliable; however, the evidence for implicit knowledge may be an artifact of the overestimation bias and should be interpreted with caution.

Taken together, when considering the limitations discovered in our studies, the PD approach to using the generation task as a measure of implicit and explicit sequence knowledge in the SRTT has so far yielded few reliable conclusions. If anything, results support the presence of explicit knowledge and call into question the interpretation of PD results as indicative of implicit knowledge.

It might be possible to devise a version of the generation task that allows for the separation of automatic and controlled processes but does not depend on exclusion of explicit knowledge and does not induce different response criteria. For example, D'Angelo, Milliken, Jiménez, and Lupiáñez (2013) implemented such a generation task variant in artificial grammar learning in which two different inclusion instructions were compared: After learning about two different grammars, participants were asked, in the first (second) inclusion block to generate exemplars from the first (second) grammar. Under certain assumptions, performance differences between blocks can be interpreted as evidence for explicit controllable knowledge. Exclusion failure and different criteria presumably do not matter in this task: Participants were not instructed to exclude explicit knowledge, and it is plausible that the similarity of instructions for both generation tasks also induced comparable response criteria. As another example, in the domain of recognition memory, the PD procedure can be replaced by a source-memory task in which, instead of including versus excluding items from one of two study lists (A and B), participants are asked to indicate the source of the word (list A or list B; Buchner et al., 1997; Steffens, Buchner, Martensen, \& Erdfelder, 2000; Yu \& Bellezza, 2000). Perhaps with a similar modification, an improved generation task may prove a useful measure of sequence knowledge. Future research should also consider using alternative methods of assessing implicit and explicit knowledge (for a recent overview, see Timmermans \& Cleeremans, 2015).

One of the great benefits of multinomial models such as the PD model is that they are flexibly adaptable measurement models for studying latent cognitive processes using a wide variety of experimental paradigms (Erdfelder et al., 2009). To validate a new model, it is 
common to assess its goodness of fit, and to empirically demonstrate that its parameters can be selectively manipulated and interpreted psychologically (i.e., parameter estimates reflect targeted experimental manipulations in the predicted manner; W. H. Batchelder \& Riefer, 1999). In many cases, however, simplifying assumptions need to be made; for instance, latent processes are equated across two or more experimental conditions (e.g., a single controlled process $C$ is assumed to operate under inclusion and exclusion conditions). Whenever such assumptions of invariance are made, we propose that they should also be tested empirically as part of the model-validation effort when a new model is proposed, before it is used to investigate substantive issues (for an example, see Brainerd, Reyna, \& Mojardin, 1999). 


\section{References}

Albert, J. H., \& Chib, S. (1993). Bayesian Analysis of Binary and Polychotomous Response Data. Journal of the American Statistical Association, 88(422), 669-679. doi:10.1080/01621459.1993.10476321

Aust, F., \& Barth, M. (2018). papaja: Create APA manuscripts with R Markdown. Retrieved from https://github.com/crsh/papaja

Batchelder, W. H., \& Riefer, D. M. (1999). Theoretical and empirical review of multinomial process tree modeling. Psychonomic Bulletin \& Review, 6(1), 57-86. doi:10.3758/BF03210812

Brainerd, C. J., Reyna, V. F., \& Mojardin, A. H. (1999). Conjoint recognition. Psychological Review, 106(1), 160-179. doi:10.1037/0033-295X.106.1.160

Buchner, A., Erdfelder, E., \& Vaterrodt-Plünnecke, B. (1995). Toward unbiased measurement of conscious and unconscious memory processes within the process dissociation framework. Journal of Experimental Psychology: General, 124(2), 137-160. doi:10.1037/0096-3445.124.2.137

Buchner, A., Erdfelder, E., Steffens, M. C., \& Martensen, H. (1997). The nature of memory processes underlying recognition judgments in the process dissociation procedure. Memory \& Cognition, 25(4), 508-517. doi:10.3758/BF03201126

Buchner, A., Steffens, M. C., \& Rothkegel, R. (1998). On the role of fragmentary knowledge in a sequence learning task. The Quarterly Journal of Experimental Psychology Section A, 51(2), 251-281. doi:10.1080/713755757

Carpenter, B., Gelman, A., Hoffman, M., Lee, D., Goodrich, B., Betancourt, M., ... Riddell, A. (2016). Stan: A probabilistic programming language. Journal of Statistical Software, 20, 1-37. doi:10.18637/jss.v076.i01

Cleeremans, A., \& Jiménez, L. (2002). Implicit learning and consciousness: A graded, dynamic perspective. In R. M. French \& A. Cleeremans (Eds.), Implicit learning and consciousness: An empirical, philosophical and computational consensus in the making (pp. 1-40). Hove: Psychology Press. Retrieved from http://journalpsyche. org/articles/0xc03a.pdf

Cohen, A., Ivry, R. I., \& Keele, S. W. (1990). Attention and structure in sequence learning. Journal of Experimental Psychology: Learning, Memory, and Cognition, 16(1), 17-30. doi:10.1037/0278-7393.16.1.17

Curran, T. (2001). Implicit learning revealed by the method of opposition. Trends in Cognitive Sciences, 5(12), 503-504. doi:10.1016/S1364-6613(00)01791-5

Curran, T., \& Hintzman, D. L. (1995). Violations of the independence assumption in process dissociation. Journal of Experimental Psychology: Learning, Memory, and Cognition, 
21(3), 531-547. doi:10.1037/0278-7393.21.3.531

Curran, T., \& Hintzman, D. L. (1997). Consequences and causes of correlations in process dissociation. Journal of Experimental Psychology. Learning, Memory 83 Cognition, 23(2), 496. doi:10.1037/0278-7393.23.2.496

Destrebecqz, A. (2004). The effect of explicit knowledge on sequence learning: A graded account. Psychologica Belgica, 44(4), 217. doi:10.5334/pb-44-4-217

Destrebecqz, A., \& Cleeremans, A. (2001). Can sequence learning be implicit? New evidence with the process dissociation procedure. Psychonomic Bulletin $\&$ Review, 8(2), 343-350. doi:10.3758/BF03196171

Destrebecqz, A., \& Cleeremans, A. (2003). Temporal effects in sequence learning. In Attention and implicit learning (pp. 181-213). Amsterdam, Netherlands: John Benjamins Publishing Company. doi:10.1075/aicr.48.11des

D’Angelo, M. C., Milliken, B., Jiménez, L., \& Lupiáñez, J. (2013). Implementing flexibility in automaticity: Evidence from context-specific implicit sequence learning. Consciousness and Cognition, 22(1), 64-81. doi:10.1016/j.concog.2012.11.002

Erdfelder, E., Auer, T.-S., Hilbig, B. E., Aßfalg, A., Moshagen, M., \& Nadarevic, L. (2009). Multinomial Processing Tree Models: A review of the literature. Zeitschrift Für Psychologie / Journal of Psychology, 217(3), 108-124. doi:10.1027/0044-3409.217.3.108

Fu, Q., Fu, X., \& Dienes, Z. (2008). Implicit sequence learning and conscious awareness. Consciousness and Cognition, 17(1), 185-202. doi:10.1016/j.concog.2007.01.007

Graf, P., \& Komatsu, S.-I. (1994). Process dissociation procedure: Handle with caution! European Journal of Cognitive Psychology, 6(2), 113-129. doi:10.1080/09541449408520139

Haider, H., \& Frensch, P. A. (2009). Conflicts between expected and actually performed behavior lead to verbal report of incidentally acquired sequential knowledge. Psychological Research, 73(6), 817-834. doi:10.1007/s00426-008-0199-6

Haider, H., Eichler, A., \& Lange, T. (2011). An old problem: How can we distinguish between conscious and unconscious knowledge acquired in an implicit learning task? Consciousness and Cognition, 20(3), 658-672. doi:10.1016/j.concog.2010.10.021

Hintzman, D. L., \& Curran, T. (1997). More than one way to violate independence: Reply to Jacoby and Shrout (1997). Journal of Experimental Psychology. Learning, Memory E Cognition, 23(2), 511. doi:10.1037/0278-7393.23.2.511

Hirshman, E. (2004). Ordinal Process Dissociation and the Measurement of Automatic and Controlled Processes. Psychological Review, 111(2), 553-560. doi:10.1037/0033295X.111.2.553

Jacoby, L. L. (1991). A process dissociation framework: Separating automatic from intentional uses of memory. Journal of Memory and Language, 30(5), 513-541. 
doi:10.1016/0749-596X(91)90025-F

Jacoby, L. L., \& Shrout, P. E. (1997). Toward a psychometric analysis of violations of the independence assumption in process dissociation. Journal of Experimental Psychology. Learning, Memory \& Cognition, 23(2), 505. doi:10.1037/0278-7393.23.2.505

Jacoby, L. L., Begg, I. M., \& Toth, J. P. (1997). In defense of functional independence: Violations of assumptions underlying the... Journal of Experimental Psychology. Learning, Memory $\&$ Cognition, 23(2), 484. Retrieved from http://search.ebscohost. com/login.aspx $?$ direct $=$ true $\& d b=b t h \& A N=9706175953 \&$ site $=$ ehost-live

Jacoby, L. L., Toth, J. P., \& Yonelinas, A. P. (1993). Separating conscious and unconscious influences of memory: Measuring recollection. Journal of Experimental Psychology: General, 122(2), 139-154. doi:10.1037/0096-3445.122.2.139

Jiménez, L., \& Méndez, C. (1999). Which attention is needed for implicit sequence learning? Journal of Experimental Psychology: Learning, Memory, and Cognition, 25(1), 236-259. doi:10.1037/0278-7393.25.1.236

Jiménez, L., Méndez, C., \& Cleeremans, A. (1996). Comparing direct and indirect measures of sequence learning. Journal of Experimental Psychology: Learning, Memory, and Cognition, 22(4), 948. doi:10.1037/0278-7393.22.4.948

Jiménez, L., Vaquero, J. M. M., \& Lupiáñez, J. (2006). Qualitative differences between implicit and explicit sequence learning. Journal of Experimental Psychology: Learning, Memory, and Cognition, 32(3), 475-490. doi:10.1037/0278-7393.32.3.475

Joordens, S., \& Merikle, P. M. (1993). Independence or redundancy? Two models of conscious and unconscious influences. Journal of Experimental Psychology: General, 122(4), 462-467. doi:10.1037/0096-3445.122.4.462

Joordens, S., Wilson, D. E., Spalek, T. M., \& Paré, D. E. (2010). Turning the processdissociation procedure inside-out: A new technique for understanding the relation between conscious and unconscious influences. Consciousness and Cognition, 19(1), 270-280. doi:10.1016/j.concog.2009.09.011

Klauer, K. C. (2010). Hierarchical Multinomial Processing Tree Models: A Latent-Trait Approach. Psychometrika, 75(1), 70-98. doi:10.1007/s11336-009-9141-0

Klauer, K. C., Dittrich, K., Scholtes, C., \& Voss, A. (2015). The Invariance Assumption in Process-Dissociation Models: An Evaluation Across Three Domains. Journal of Experimental Psychology: General, 144(1), 198-221. doi:10.1037/xge0000044

Knapp, B. R., \& Batchelder, W. H. (2004). Representing parametric order constraints in multi-trial applications of multinomial processing tree models. Journal of Mathematical Psychology, 48(4), 215-229. doi:10.1016/j.jmp.2004.03.002

Koch, I., \& Hoffmann, J. (2000). Patterns, chunks, and hierarchies in serial reaction-time 
tasks. Psychological Research, 63(1), 22-35. doi:10.1007/PL00008165

Lewandowski, D., Kurowicka, D., \& Joe, H. (2009). Generating random correlation matrices based on vines and extended onion method. Journal of Multivariate Analysis, 100 (9), 1989-2001. doi:10.1016/j.jmva.2009.04.008

Liefooghe, B., Wenke, D., \& De Houwer, J. (2012). Instruction-based task-rule congruency effects. Journal of Experimental Psychology: Learning, Memory, and Cognition, 38(5), 1325-1335. doi:10.1037/a0028148

Nissen, M. J., \& Bullemer, P. (1987). Attentional requirements of learning: Evidence from performance measures. Cognitive Psychology, 19(1), 1-32. doi:10.1016/00100285(87)90002-8

Norman, E., Price, M. C., \& Duff, S. C. (2006). Fringe consciousness in sequence learning: The influence of individual differences. Consciousness and Cognition, 15(4), 723-760. doi:10.1016/j.concog.2005.06.003

Perruchet, P., \& Amorim, M.-A. (1992). Conscious knowledge and changes in performance in sequence learning: Evidence against dissociation. Journal of Experimental Psychology: Learning, Memory, and Cognition, 18(4), 785. doi:10.1037/0278-7393.18.4.785

Perruchet, P., \& Gallego, J. (1993). Association between conscious knowledge and performance in normal subjects: Reply to Cohen and Curran (1993) and Willingham, Greeley, and Bardone (1993). doi:10.1037/0278-7393.19.6.1438

Perruchet, P., Bigand, E., \& Benoit-Gonin, F. (1997). The emergence of explicit knowledge during the early phase of learning in sequential reaction time tasks. Psychological Research, 60(1-2), 4-13. doi:10.1007/BF00419676

R Core Team. (2017). R: A language and environment for statistical computing. Vienna, Austria: R Foundation for Statistical Computing. Retrieved from https://www. R-project.org/

Ratcliff, R., Van Zandt, T., \& McKoon, G. (1995). Process dissociation, single-process theories, and recognition memory. Journal of Experimental Psychology: General, 124 (4), 352-374. doi:10.1037/0096-3445.124.4.352

Reed, J., \& Johnson, P. (1994). Assessing implicit learning with indirect tests: Determining what is learned about sequence structure. Journal of Experimental Psychology: Learning, Memory, and Cognition, 20(3), 585-594. doi:10.1037/0278-7393.20.3.585

Reingold, E. M., \& Merikle, P. M. (1990). On the inter-relatedness of theory and measurement in the study of unconscious processes. Mind E Language, 5(1), 9-28. doi:10.1111/j.1468-0017.1990.tb00150.x

Rouder, J. N., \& Lu, J. (2005). An introduction to Bayesian hierarchical models with an application in the theory of signal detection. Psychonomic Bulletin \& Review, 12(4), 
573-604. doi:10.3758/BF03196750

Rouder, J. N., Lu, J., Morey, R. D., Sun, D., \& Speckman, P. L. (2008). A hierarchical process-dissociation model. Journal of Experimental Psychology: General, 137(2), 370-389. doi:10.1037/0096-3445.137.2.370

Shanks, D. R., \& Johnstone, T. (1999). Evaluating the relationship between explicit and implicit knowledge in a sequential reaction time task. Journal of Experimental Psychology: Learning, Memory, and Cognition, 25(6), 1435-1451. doi:10.1037/02787393.25.6.1435

Shanks, D. R., \& Perruchet, P. (2002). Dissociation between priming and recognition in the expression of sequential knowledge. Psychonomic Bulletin \& Review, 9(2), 362-367. doi:10.3758/BF03196294

Shanks, D. R., \& St. John, M. F. (1994). Characteristics of dissociable human learning systems. Behavioral and Brain Sciences, 17(3), 367-395. doi:10.1017/S0140525X00035032

Shanks, D. R., Rowland, L. A., \& Ranger, M. S. (2005). Attentional load and implicit sequence learning. Psychological Research, 69(5-6), 369-382. doi:10.1007/s00426004-0211-8

Singmann, H., Bolker, B., Westfall, J., \& Aust, F. (2018). Afex: Analysis of factorial experiments. Retrieved from https://CRAN.R-project.org/package=afex

Spiegelhalter, D. J., Best, N. G., Carlin, B. P., \& Van Der Linde, A. (2002). Bayesian measures of model complexity and fit. Journal of the Royal Statistical Society: Series B (Statistical Methodology), 64(4), 583-639. doi:10.1111/1467-9868.00353

Spiegelhalter, D. J., Best, N. G., Carlin, B. P., \& van der Linde, A. (2014). The deviance information criterion: 12 years on. Journal of the Royal Statistical Society: Series B (Statistical Methodology), 76(3), 485-493. doi:10.1111/rssb.12062

Stahl, C., Barth, M., \& Haider, H. (2015). Distorted estimates of implicit and explicit learning in applications of the process-dissociation procedure to the SRT task. Consciousness and Cognition, 37, 27-43. doi:10.1016/j.concog.2015.08.003

Steffens, M. C., Buchner, A., Martensen, H., \& Erdfelder, E. (2000). Further evidence on the similarity of memory processes in the process dissociation procedure and in source monitoring. Memory \& Cognition, 28(7), 1152-1164. doi:10.3758/BF03211816

Timmermans, B., \& Cleeremans, A. (2015). How can we measure awareness? An overview of current methods. In Behavioural Methods in Consciousness Research (pp. 21-46). Oxford: Oxford University Press.

Toth, J. P., Reingold, E. M., \& Jacoby, L. L. (1994). Toward a redefinition of implicit memory: Process dissociations following elaborative processing and self-generation. Journal of Experimental Psychology: Learning, Memory, and Cognition, 20(2), 
290-303. doi:10.1037/0278-7393.20.2.290

Wilkinson, L., \& Shanks, D. R. (2004). Intentional control and implicit sequence learning. Journal of Experimental Psychology: Learning, Memory, and Cognition, 30(2), 354-369. doi:10.1037/0278-7393.30.2.354

Willingham, D. B., Nissen, M. J., \& Bullemer, P. (1989). On the development of procedural knowledge. Journal of Experimental Psychology: Learning, Memory, and Cognition, 15(6), 1047-1060. doi:10.1037/0278-7393.15.6.1047

Yu, J., \& Bellezza, F. S. (2000). Process dissociation as source monitoring. Journal of Experimental Psychology: Learning, Memory, and Cognition, 26(6), 1518-1533. doi:10.1037/0278-7393.26.6.1518 
Appendix A

Generation performance

This appendix provides the raw generation performance for all experiments in tables A1, $\mathrm{A} 2$, and $\mathrm{A} 3$.

Table A1

Mean percentage of regular transitions generated in Experiment 1, excluding repetions. Standard deviations are given in parentheses.

\begin{tabular}{lcl}
\hline & Inclusion & Exclusion \\
\hline Full dataset & & \\
Control & $25.10(11.74)$ & $24.17(7.02)$ \\
No-Practice & $37.94(16.26)$ & $28.66(13.39)$ \\
Unspecific-Practice & $34.46(14.14)$ & $26.46(15.02)$ \\
Practice & $38.74(13.08)$ & $24.59(9.34)$ \\
Transfer & $56.16(18.32)$ & $26.51(7.93)$ \\
Nonrevealed transitions & & \\
Control & $25.10(11.74)$ & $24.17(7.02)$ \\
No-Practice & $29.20(18.56)$ & $31.90(14.01)$ \\
Unspecific-Practice & $30.38(15.48)$ & $29.34(14.06)$ \\
Practice & $29.63(14.62)$ & $26.81(11.35)$ \\
Transfer & $45.68(24.66)$ & $43.95(17.03)$ \\
Revealed, but nonpracticed transitions & & \\
No-Practice & $47.64(39.71)$ & $24.65(31.82)$ \\
Unspecific-Practice & $33.91(32.58)$ & $20.07(26.96)$ \\
Transfer & $59.65(33.59)$ & $16.72(22.52)$ \\
Revealed-and-practiced transitions & & \\
Practice & $75.65(24.96)$ & $15.63(29.87)$ \\
Transfer & $79.51(21.81)$ & $7.50(7.13)$ \\
\hline
\end{tabular}




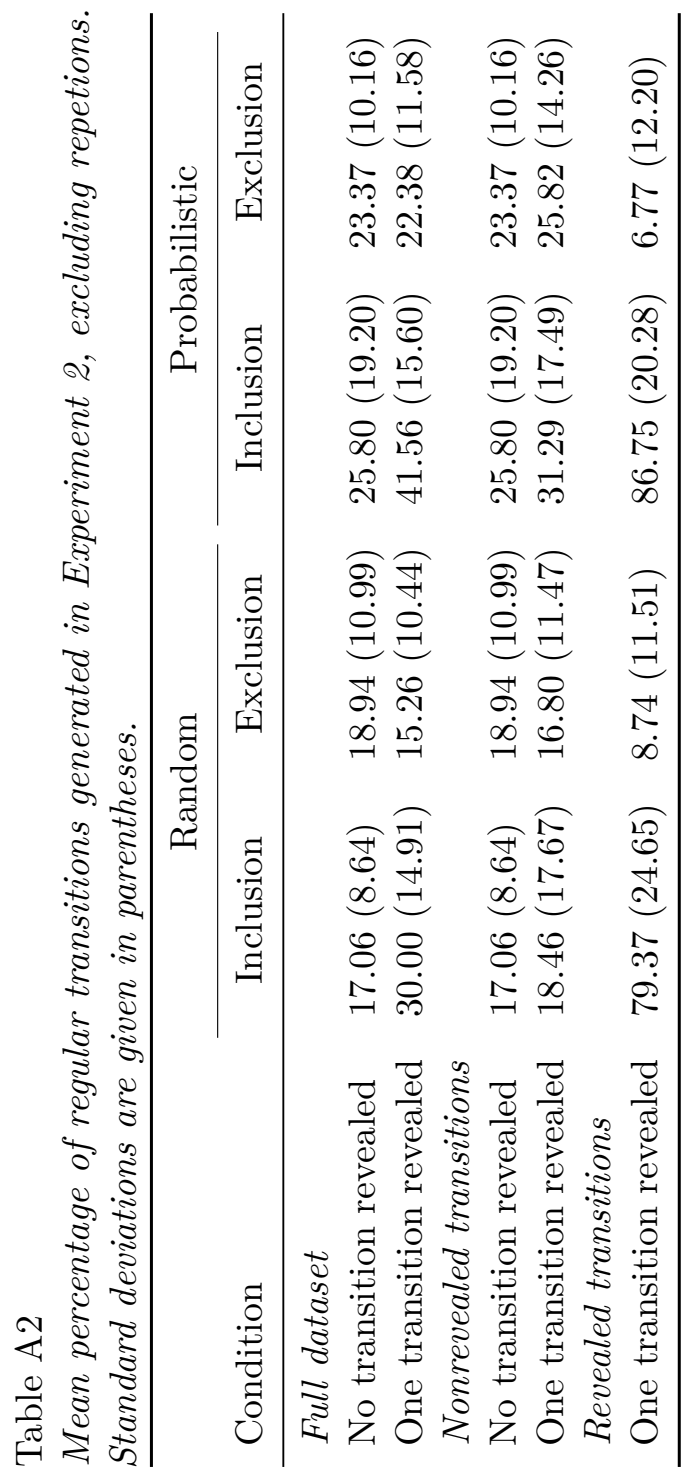




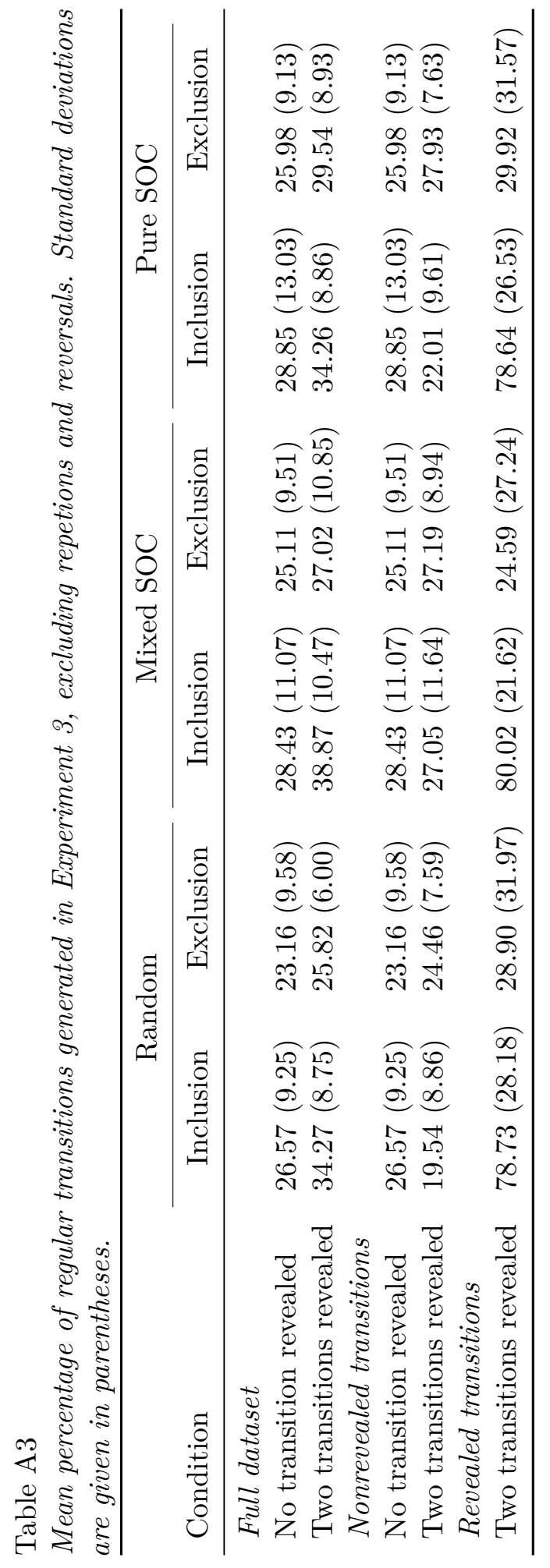


Appendix B

Additional ordinal-PD analyses

This appendix provides results of additional ordinal-PD analyses for Experiments 2 and 3 .

\section{Experiment 2}

Figure B1 shows the overall generation performance. We conducted a 2 (Material: Random vs. Probabilistic) $\times 2$ (Explicit knowledge: No transition revealed vs. One transition revealed $) \times 2$ (Block order: Inclusion first vs. Exclusion first $) \times 2(P D$ instruction: Inclusion vs. Exclusion) ANOVA that revealed a main effect of $P D$ instruction, $F(1,113)=28.43$, $M S E=156.22, p<.001, \hat{\eta}_{G}^{2}=.109$, participants generated more regular transitions in inclusion than exclusion blocks; and a main effect of explicit knowledge, $F(1,113)=13.00$, $M S E=164.96, p<.001, \hat{\eta}_{G}^{2}=.056$, indicating a clear influence of the explicit knowledge manipulation on generation performance. Moreover, we found a main effect of material, $F(1,113)=22.95, M S E=164.96, p<.001, \hat{\eta}_{G}^{2}=.094$, participants generated more regular transitions if they had worked on regular material during the SRTT; the effect of block order also trended to be significant, $F(1,113)=3.57, M S E=164.96, p=.062, \hat{\eta}_{G}^{2}=.016$, participants generated slightly more regular transitions if inclusion followed exclusion. These main effects were qualified by two-way interactions of explicit knowledge and block order, $F(1,113)=10.31, M S E=164.96, p=.002, \hat{\eta}_{G}^{2}=.045 ;$ and of explicit knowledge and $P D$ instruction, $F(1,113)=26.64, M S E=156.22, p<.001, \hat{\eta}_{G}^{2}=.103$; moreover, the four-way interaction of material, explicit knowledge, block order, and PD instruction was also found to be significant, $F(1,113)=5.42, M S E=156.22, p=.022, \hat{\eta}_{G}^{2}=.023$. To disentangle these interactions, we analyzed inclusion and exclusion performance, separately.

Inclusion. Analyzing the number of regular transitions generated in inclusion blocks, a 2 (Material: Random vs. Probabilistic) $\times 2$ (Explicit knowledge: No transition revealed vs. One transition revealed $) \times 2$ (Block order: Inclusion first vs. Exclusion first) ANOVA revealed a main effect of material, $F(1,113)=14.72, M S E=207.66, p<.001, \hat{\eta}_{G}^{2}=.115$, participants generated more regular transitions if they had worked on probabilistic materials; and a main effect of explicit knowledge, $F(1,113)=29.57, M S E=207.66, p<.001$, $\hat{\eta}_{G}^{2}=.207$, indicating a clear influence of our explicit-knowledge manipulation on inclusion performance. This effect was qualified by a significant interaction of explicit knowledge and block order, $F(1,113)=9.64, M S E=207.66, p=.002, \hat{\eta}_{G}^{2}=.079$, indicating that participants used their explicit sequence knowledge more extensively if inclusion followed exclusion (i.e., after we had represented the transition a second time).

Exclusion. Analyzing the number of regular transitions generated in exclusion blocks, a 2 (Material: Random vs. Probabilistic) $\times 2$ (Explicit knowledge: No transition revealed vs. One transition revealed $) \times 2$ (Block order: Inclusion first vs. Exclusion first) ANOVA revealed a main effect of material $F(1,113)=8.87, M S E=113.52, p=.004$, $\hat{\eta}_{G}^{2}=.073$, participants generated more regular transitions if they had worked on probabilistic materials during the SRTT. We also found a significant three-way interaction of material, explicit knowledge, and block order, $F(1,113)=4.21, M S E=113.52, p=.042, \hat{\eta}_{G}^{2}=.036$ : Exclusion performance was below baseline only if exclusion followed inclusion and participants had worked on random material during the SRTT (i.e., they only had knowledge about one single transition of the sequence and had maximum practice in including/excluding this 

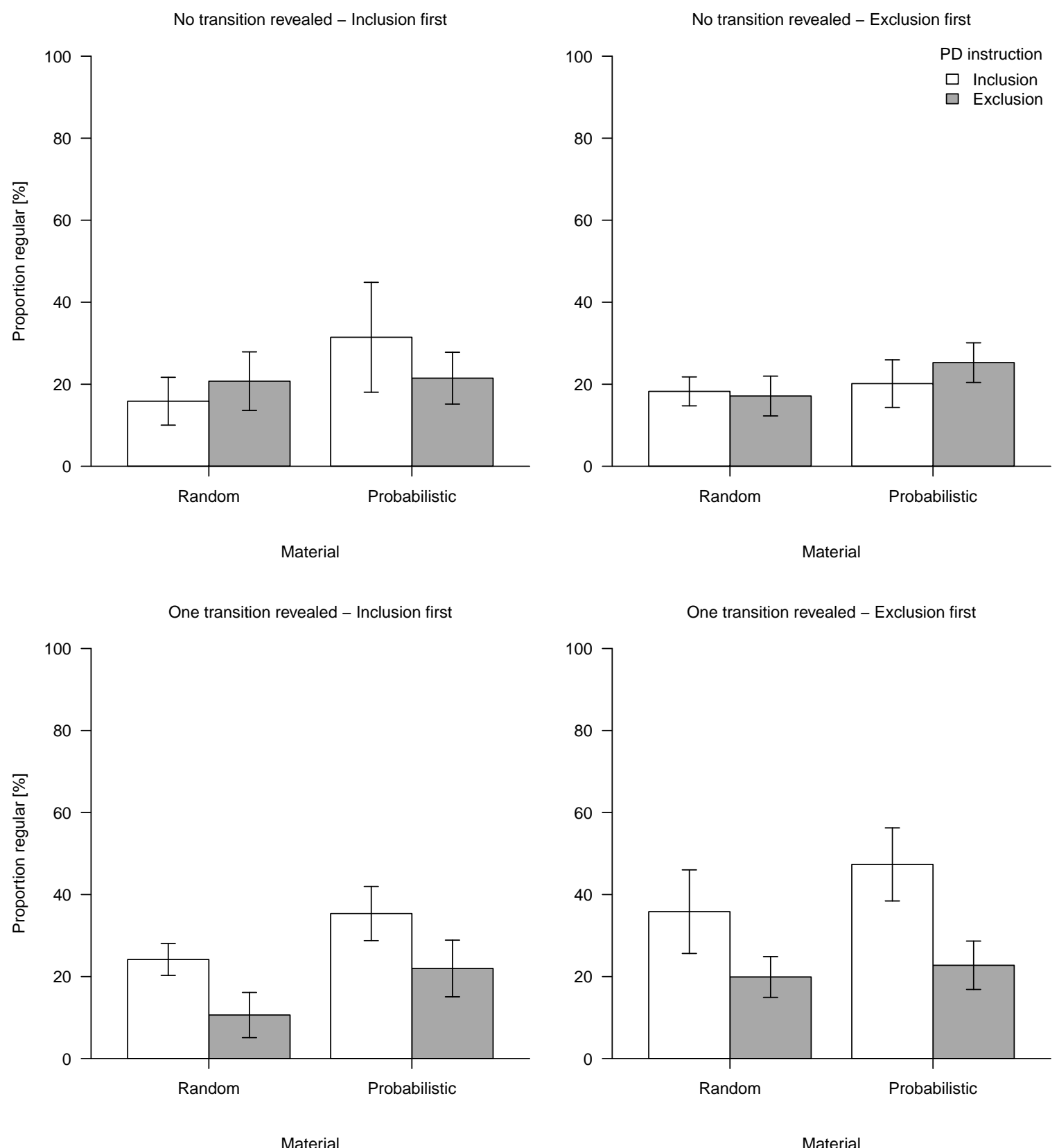

Figure B1. Mean proportion of correct FOCs during the generation task of Experiment 2, excluding repetitions. Error bars represent 95\% confidence intervals. 

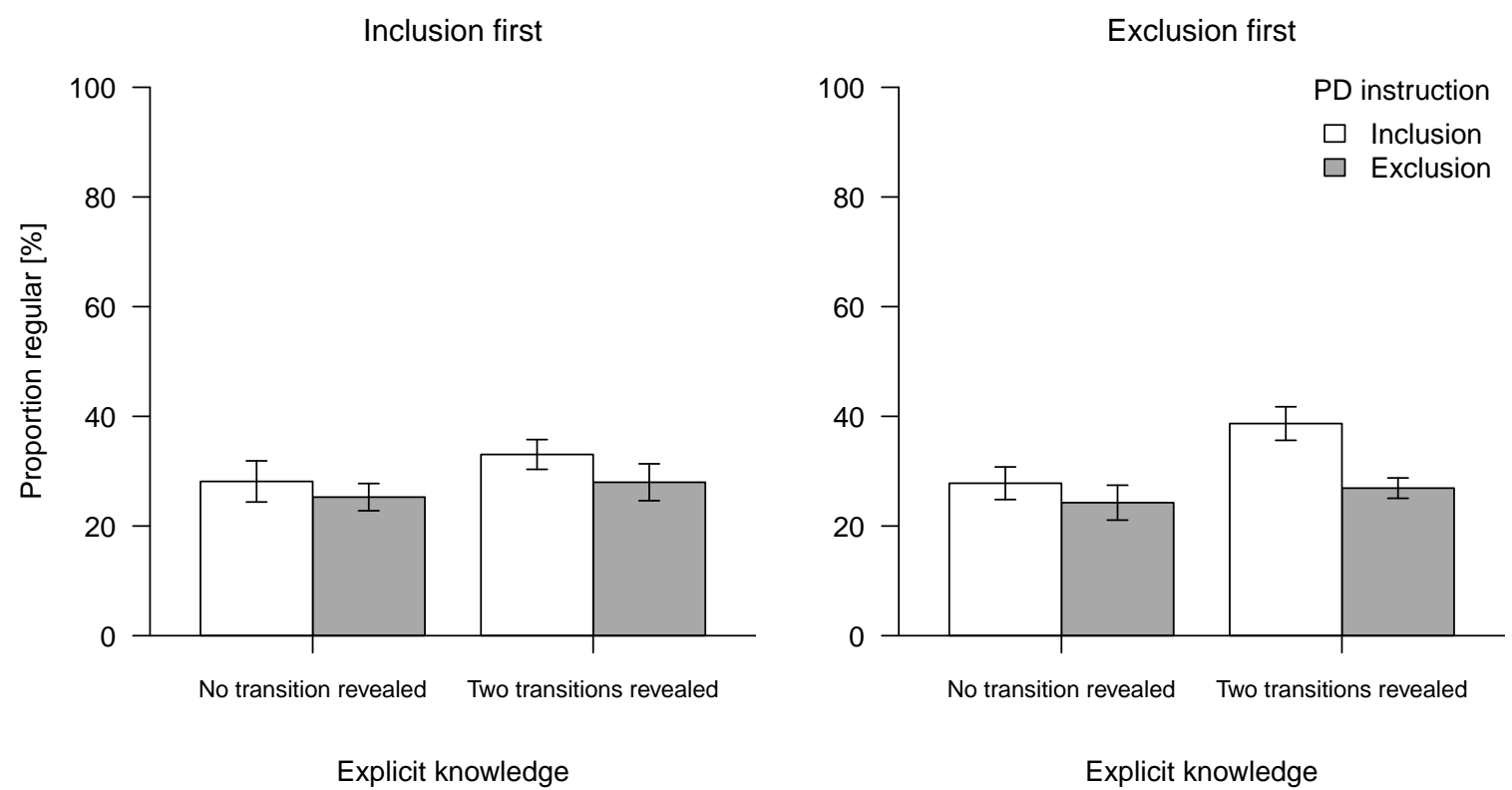

Figure B2. Mean proportion of correct SOCs during the generation task of Experiment 3, excluding repetitions and reversals. Error bars represent $95 \%$ confidence intervals.

transition) - that is, if participants had no sequence knowledge but the single transition that we had revealed to them and they had already used this knowledge during the inclusion block, they were able to generate less regular transitions than baseline during the following exclusion block. The monotonicity assumption of the ordinal-PD approach is thus not violated in this single cell of the design. It is, hoewever, violated if exclusion preceded inclusion, or if participants had worked on probabilistic materials.

\section{Experiment 3}

Figure B2 shows the overall generation performance. A 3 (Material: Random vs. mixed SOC vs. pure SOC $) \times 2$ (Explicit knowledge: No transition revealed vs. Two transitions revealed $) \times 2($ Block Order: Inclusion first vs. Exclusion first $) \times 2(P D$ instruction: Inclusion vs. Exclusion) ANOVA revealed a main effect of $P D$ instruction, $F(1,159)=30.61, M S E=$ $94.53, p<.001, \hat{\eta}_{G}^{2}=.087$, participants generated more regular transitions in inclusion than exclusion blocks; and a main effect of explicit knowledge, $F(1,159)=25.01, M S E=97.20$, $p<.001, \hat{\eta}_{G}^{2}=.074$, indicating a clear influence of the explicit knowledge manipulation on generation performance. Moreover, the interaction of explicit knowledge and PD instruction reached significance, $F(1,159)=6.18, M S E=94.53, p=.014, \hat{\eta}_{G}^{2}=.019$, indicating that the effect of explicit knowledge is qualified by $P D$ instruction. The interaction of $P D$ instruction and block order almost reached significance, $F(1,159)=3.04, M S E=94.53$, $p=.083, \hat{\eta}_{G}^{2}=.009$. To disentangle these interactions, we analyzed inclusion and exclusion performance, separately.

Inclusion. Analyzing the number of regular transitions generated in inclusion blocks, a 3 (Material: Random vs. mixed SOC vs. pure SOC) $\times 2$ (Explicit knowledge: No transition revealed vs. Two transitions revealed) $\times 2$ (Block Order: Inclusion first vs. Exclusion 
first) ANOVA revealed a significant main effect of explicit knowledge, $F(1,159)=25.27$, $M S E=106.81, p<.001, \hat{\eta}_{G}^{2}=.137$, indicating that our manipulation of explicit knowledge influenced inclusion performance. The main effect of block order trended to be significant, $F(1,159)=2.84, M S E=106.81, p=.094, \hat{\eta}_{G}^{2}=.018$, which was qualified by an almost significant interaction of explicit knowledge and block order, $F(1,159)=3.70, M S E=106.81$, $p=.056, \hat{\eta}_{G}^{2}=.023$. This pattern indicated that more regular transitions were generated if participants had received explicit knowledge about two transitions and inclusion followed exclusion, i.e. the explicit knowledge had been presented a second time (once prior to exclusion, once prior to inclusion).

Exclusion. Analyzing the number of regular transitions generated in exclusion blocks, a 3 (Material: Random vs. mixed SOC vs. pure SOC) $\times 2$ (Explicit knowledge: No transition revealed vs. Two transitions revealed) $\times 2$ (Block Order: Inclusion first vs. Exclusion first) ANOVA revealed only an almost significant main effect of explicit knowledge, $F(1,159)=3.72, M S E=84.92, p=.056, \hat{\eta}_{G}^{2}=.023$; revealing explicit knowledge about the sequence slightly increased the proportion of regular transitions generated. This pattern, again, violates the core assumption of the ordinal-PD approach that increasing amounts of explicit knowledge monotonically decrease the proportion of regular transitions in exclusion blocks. Moreover, it also shows that increasing explicit knowledge might produce a data pattern that is typically interpreted as evidence for increasing amounts of implicit knowldge.

\section{Appendix C}

Additional model analyses

This appendix provides results of additional model analyses not included in the main text.

\section{Experiment 1, model $\mathcal{M}_{1}$}

In Experiment 1, we fitted model $\mathcal{M}_{1}$ and used posterior analyses to evaluate the invariance assumption. We adapted the equations from Experiment 2 to the design of Experiment 1 (which did not contain experimental groups with random material). In order to accommodate for the more complex design, we used a model specification that allowed for participant and item (i.e., transition) effects and their interactions by estimating fixed effects for each transition type plus individual participants' deviations from these effects. The model equations of model $\mathcal{M}_{1}$ are given by:

$C_{i j m}= \begin{cases}\Phi\left(\mu_{j l m}^{(C)}+\delta_{i j m}^{(C)}\right) & \text { if } j \epsilon 1,2 \text { (item has been revealed \& practiced, revealed \& non-practiced) } \\ 0 & \text { if } j=3 \text { (item has not been revealed) }\end{cases}$

and

$$
A_{i m t}=\Phi\left(\mu_{m t}^{(A)}+\delta_{i m t}^{(A)}\right)
$$

where $\mu_{j l m}^{(C)}$ is the fixed effect of transition type $j$ (non-revealed, revealed \& practiced, revealed $\&$ non-practiced) in condition $l$ and $P D$ instruction condition $m$ on controlled processes, and $\delta_{i j m}^{(C)}$ is the $i$ th participant's deviation from the corresponding mean. Accordingly, $\mu_{m t}^{(A)}$ is the fixed effect of $P D$ instruction condition $m$ and transition $t$ on automatic processes, and $\delta_{i m t}^{(A)}$ is the $i$ th participant's deviation from the corresponding mean. 
Model $\mathcal{M}_{1}$ imposes two auxiliary assumptions: First, it assumed that no explicit knowledge has been acquired during the SRT phase (i.e., $C=0$ for non-revealed transitions). Second, it assumed that revealing sequence knowledge did not affect automatic processes (i.e., $A$ does not vary as a function of the between-subjects manipulation of explicit knowledge, index $l$ ). Both auxiliary assumptions were tested by posterior predictive checks. In addition to reporting $T_{A 1}$ and $T_{B 1}$ as in Experiments 2 and 3, we calculated additional model check statistic $T_{A 2}$, which summarizes how well the model describes the item-wise category counts (aggregated over participants), and $T_{A 3}$, which summarizes how well the model describes the category counts per participant-item combination; finally, the additional statistic $T_{B 2}$ summarizes how well the model describes the variances and covariances introduced by items. We also calculated the posterior differences $C_{I}-C_{E}$ and $A_{I}-A_{E}$ to more directly test the invariance assumption.

Results. We analyzed generation performance by fitting $\mathcal{M}_{1}$ and computed model fit statistics to assess whether each model can account for the data. Parameter estimates from model $\mathcal{M}_{1}$ were used to address the invariance assumptions, directly. The first trial of a block as well as any response repetitions were excluded from all generation task analyses.

The model checks for model $\mathcal{M}_{1}$ were satisfactory,

$$
\begin{gathered}
T_{A 1}^{\text {observed }}=35.97, T_{A 1}^{\text {expected }}=33.96, p=.322, \\
T_{A 2}^{\text {observed }}=0.05, T_{A 2}^{\text {expected }}=0.05, p=.480, \\
T_{A 3}^{\text {observed }}=1,763.79, T_{A 3}^{\text {expected }}=1,720.63, p=.372, \\
T_{B 1}^{\text {observed }}=5.31, T_{B 1}^{\text {expected }}=4.62, p=.457, \\
T_{B 2}^{\text {observed }}=3,852.65, T_{B 2}^{\text {expected }}=3,393.90, p=.464 .
\end{gathered}
$$

Figure $\mathrm{C} 1$ shows the parameter estimates obtained from model $\mathcal{M}_{1}$; while estimates of the automatic process were only slightly above chance in both $P D$ instruction conditions, estimates of the controlled process differ strongly between $P D$ instruction conditions.

Figure $\mathrm{C} 2$ shows that the invariance assumption for automatic processes was violated with $A_{I}>A_{E}, 95 \%$ CI $[.00, .03]$, and Bayesian $p=.008$. For revealed and practiced transitions, the invariance assumption was violated with $C_{I}>C_{E}, 95 \%$ CI $[.19, .63]$ and a Bayesian $p=.001$. For revealed but non-practiced transitions, the invariance assumption was violated with $C_{I}>C_{E}, 95 \%$ CI $[.03, .31]$ and a Bayesian $p=.005$.

\section{Experiment 2, model $\mathcal{M}_{1 R}$}

To test whether our results are robust against changes in auxiliary assumptions, we fitted another model $\mathcal{M}_{1 R}$ with different auxiliary assumptions. Specifically, we dropped the assumption that $C=0$ for nonrevealed transitions and instead estimated explicit-knowledge parameters for all transitions. Instead, we imposed ordinal restrictions (Knapp \& Batchelder, 2004) as follows: In model $\mathcal{M}_{1 R}$, it is assumed that $C$ parameters are greater under inclusion 
Automatic processes $A$

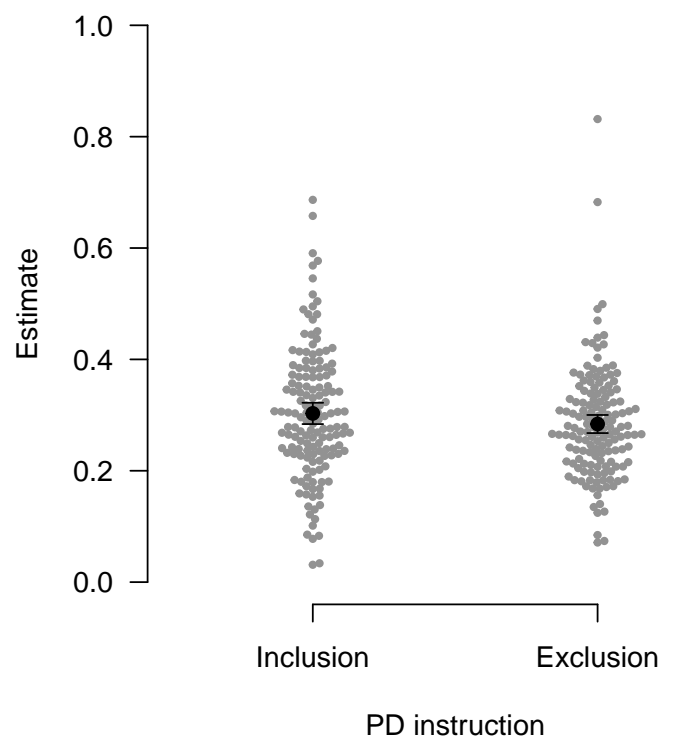

Controlled processes $C$

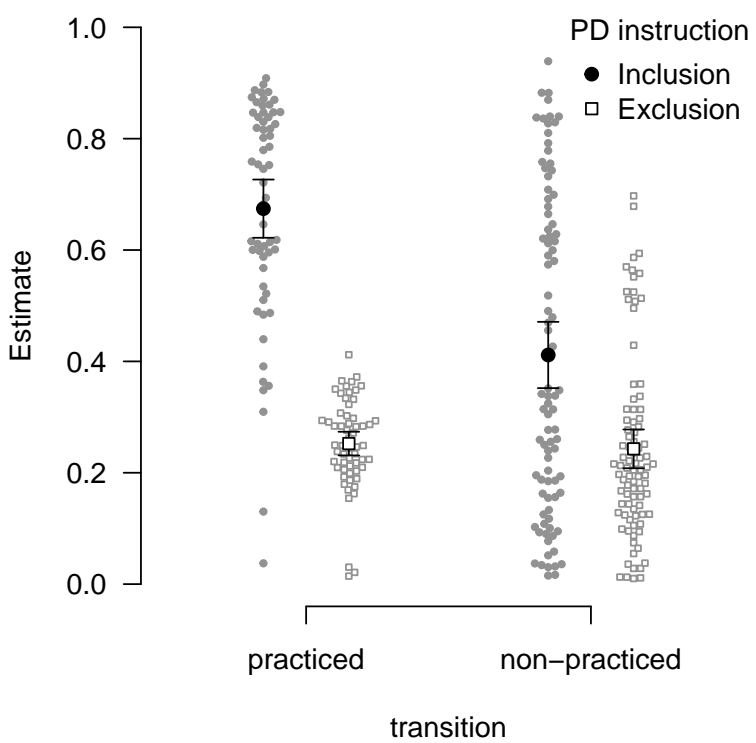

Figure $C 1$. Parameter estimates from Experiment 1, model $\mathcal{M}_{1}$. Error bars represent $95 \%$ confidence intervals.
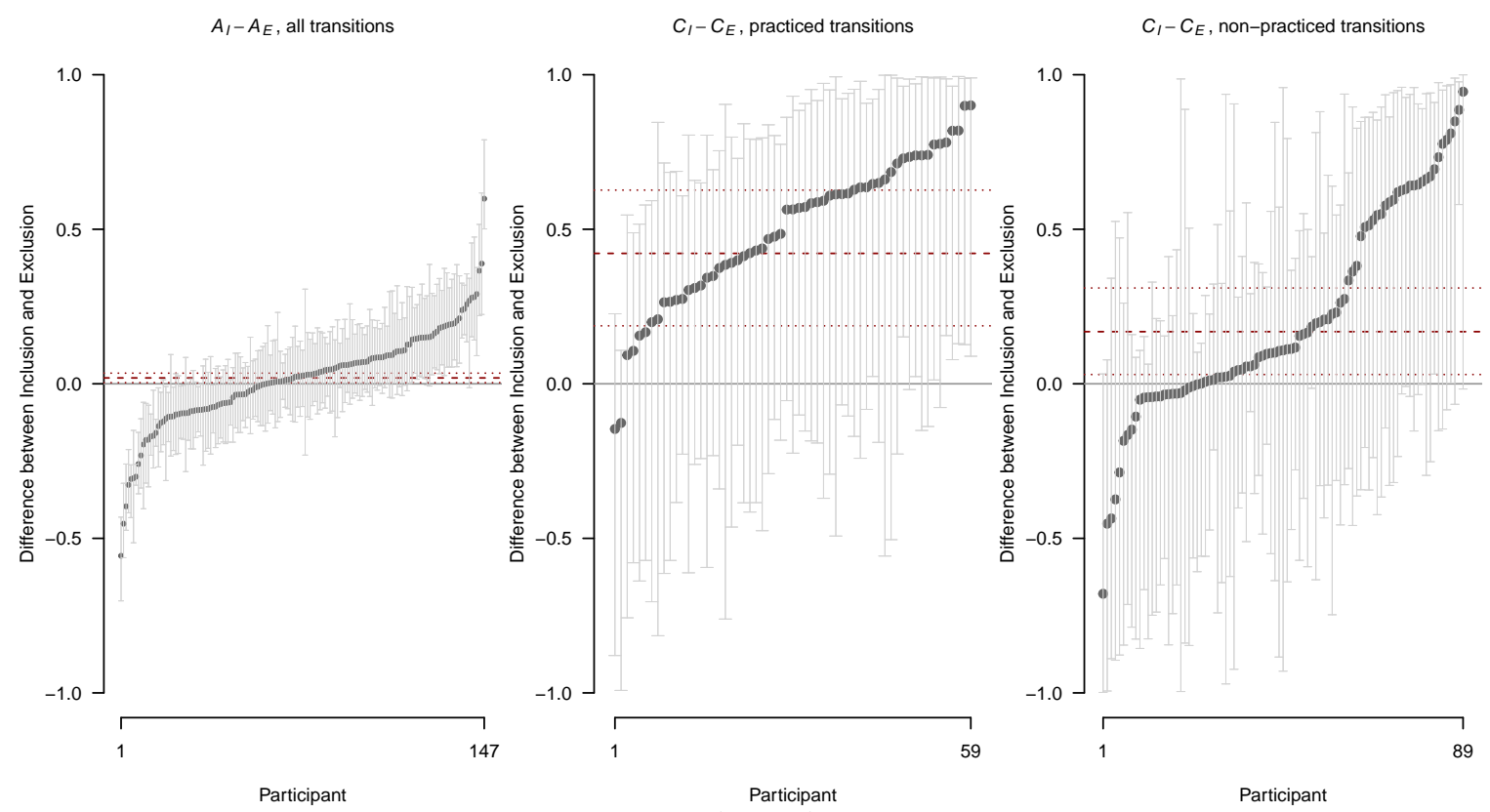

Figure C2. Posterior differences between $A_{I}-A_{E}$ and $C_{I}-C_{E}$ in Experiment 1, plotted for each participant (gray dots) with $95 \%$ credible intervals. Dashed lines represent the posterior means of the differences between mean parameter estimates. Dotted lines represent $95 \%$ credible intervals. 
than exclusion. We also fitted a parallel model with the reversed assumption, but estimation of this model failed to converge.

The second-level equations of model $\mathcal{M}_{1 R}$ are given by:

$$
\begin{aligned}
& C_{i j 1}=C_{i j, \text { Inclusion }}=\Phi\left(\mu_{j k, \text { Inclusion }}^{(C)}+\delta_{i j, \text { Inclusion }}^{(C)}\right) \\
& C_{i j 2}=C_{i j, \text { Exclusion }}=\Phi\left(\mu_{j k, \text { Exclusion }}^{(C)}+\delta_{i j, \text { Exclusion }}^{(C)}\right) * C_{i j, \text { Inclusion }}
\end{aligned}
$$

and

$$
A_{i j m}=\Phi\left(\mu_{j k m}^{(A)}+\delta_{i j m}^{(A)}\right)
$$

$\mu_{j k m}^{(C)}$ is the fixed effect of material $k$ (that participant $i$ worked on during the SRTT), transition type $j$ ( $j=1$ if a transition has actually been revealed, $j=2$ if not), and $P D$ instruction condition $m$ on controlled processes. $\delta_{i j m}^{(C)}$ is the $i$ th participant's deviation from the respective group mean. For participants who did not receive explicit knowledge about a single transition, we assumed that all $\mu_{j k, \text { Inclusion }}^{(C)}=\mu_{k, \text { Inclusion }}^{(C)}$ and $\mu_{j k, \text { Exclusion }}^{(C)}=$ $\mu_{k, \text { Exclusion }}^{(C)}$, i.e. we assumed that the grand mean of explicit knowledge did not vary as a function of the transition that would have been revealed if participants were in another condition. Accordingly, $\mu_{j k m}^{(A)}$ is the fixed effect of transition type $j(j=1$ for the transition that was or would have been revealed, i.e. transition $2-6, j=2$ for all other transitions), material $k$, and $P D$ instruction condition $m$ on automatic processes, and $\delta_{i j m}^{(A)}$ is the $i$ th participant's deviation from the corresponding mean.

Note that this specification imposes two auxiliary assumptions to the model: First, it is assumed that

$$
\forall i j\left(C_{i j, \text { Inclusion }} \geq C_{i j, \text { Exclusion }}\right)
$$

Second, it is assumed that automatic processes $A$ do not vary as a function of the betweensubjects manipulation of explicit knowledge $l$ (both assumptions were necessary so that the model was identified; an alternative model imposing an order constraint $C_{I}<C_{E}$ was also not identified).

Results. The model checks for model $\mathcal{M}_{1 R}$ were satisfactory,

$$
\begin{gathered}
T_{A 1}^{\text {observed }}=484.60, T_{A 1}^{\text {expected }}=470.11, p=.409, \\
T_{B 1}^{\text {observed }}=9.13, T_{B 1}^{\text {expected }}=6.88, p=.358 .
\end{gathered}
$$

and attained a DIC value of $25,294.53$, a value comparable to our extended model $\mathcal{M}_{1}$ presented in the main text and clearly outperforming $\mathcal{M}_{2}$. This again implies that our auxiliary assumptions introduced to $\mathcal{M}_{1 R}$ were much less problematic than the invariance assumption.

Figure C3 shows the parameter estimates obtained from model $\mathcal{M}_{1 R}$. The pattern of results mostly replicates the estimates from model $\mathcal{M}_{1}$. The main difference was that $C$ parameters were slightly greater than zero for nonrevealed transitions (these were set to zero for model $\mathcal{M}_{1}$ ). This may suggest that some explicit knowledge may have been acquired during the learning phase. Alternatively, it may also reflect a technical issue with the 

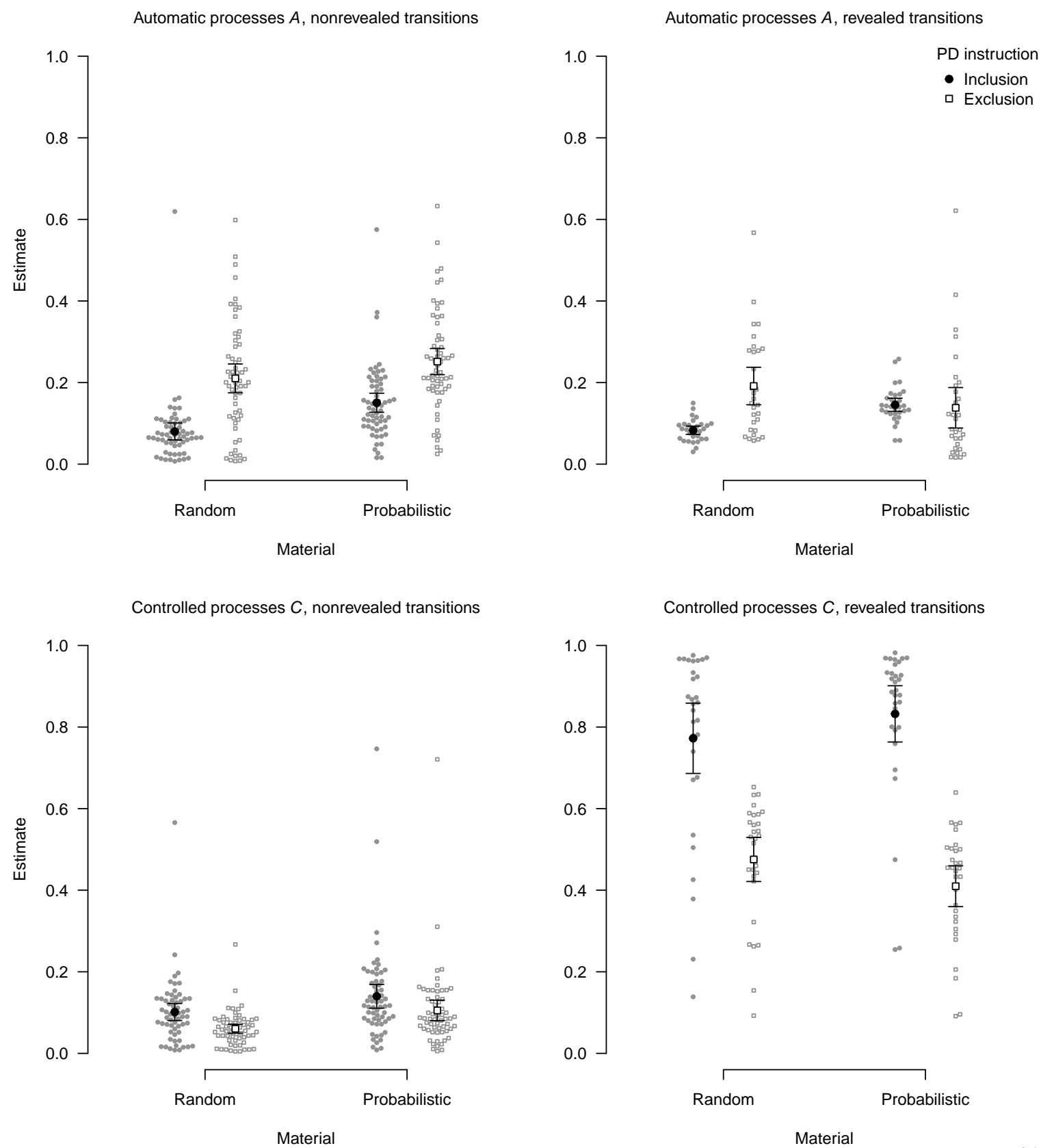

Figure C3. Parameter estimates from Experiment 2, model $\mathcal{M}_{1 R}$. Error bars represent $95 \%$ confidence intervals. 

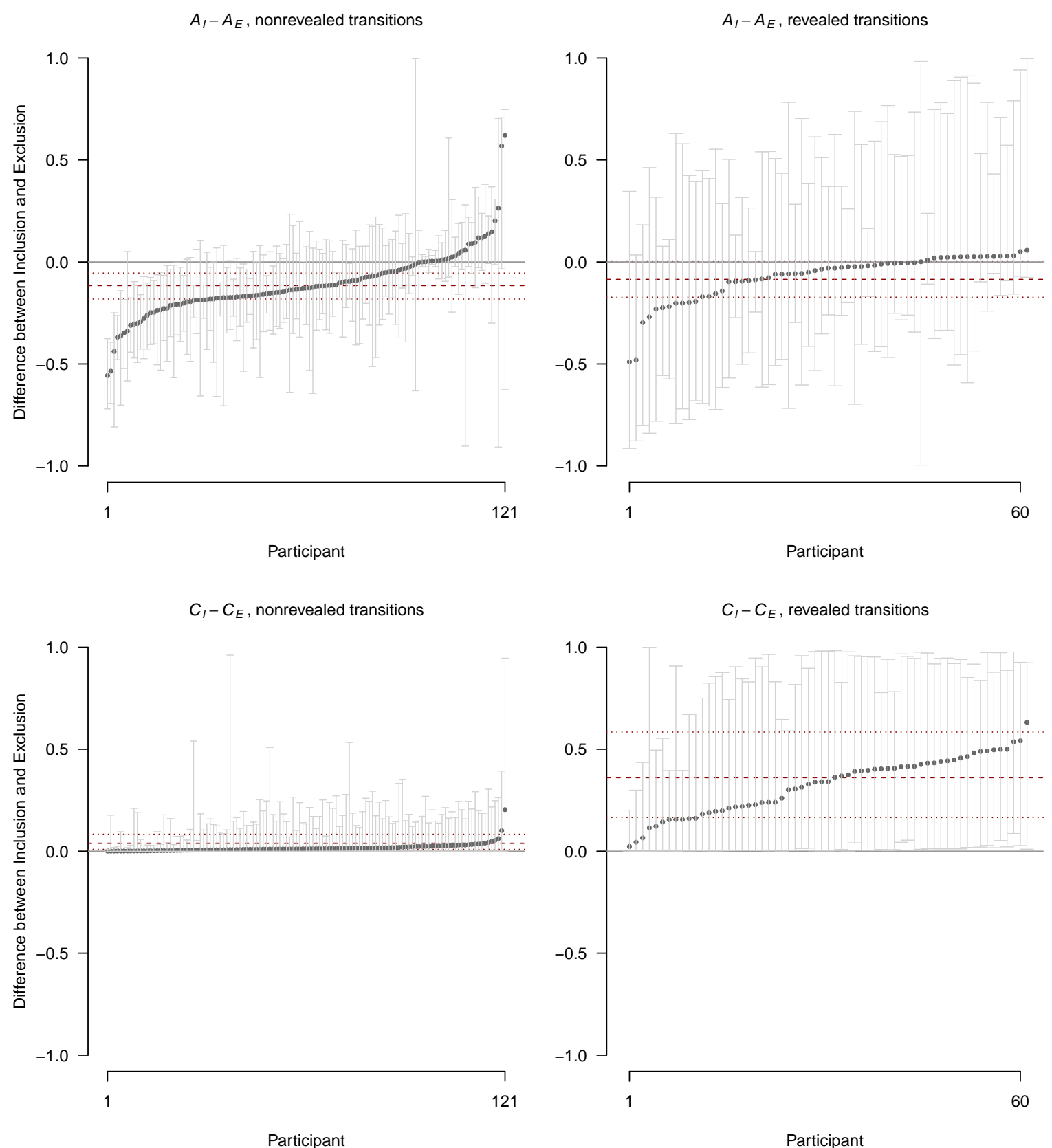

Figure $C 4$. Posterior differences between $A_{I}-A_{E}$ and $C_{I}-C_{E}$ in Experiment 2, model $\mathcal{M}_{1 R}$, plotted for each participant (gray dots) with $95 \%$ credible intervals. Dashed lines represent the posterior means of the differences between mean parameter estimates. Dotted lines represent $95 \%$ credible intervals. 
present family of models that biases estimates away from zero: Specifically, for nonrevealed transitions, the inclusion-exclusion difference in $C$ estimates should vary around zero, with half below zero and half above zero; the auxiliary assumption however forces all of them to be positive, which biases the corresponding $C$ parameters. Either way, the effect is not substantial, as suggested by the finding that model $\mathcal{M}_{1}$, which assumes $C=0$, achieved an equally good fit. The $C>0$ estimates also have a tradeoff effect on $A$ parameters, with lower estimates under inclusion and slightly higher estimates under exclusion. This biasing effect eliminated (for revealed transitions) or even inverted (for nonrevealed transitions) the invariance-violation effect found in $\mathcal{M}_{1}$.

Figure $\mathrm{C} 4$ shows the posterior differences obtained from model $\mathcal{M}_{1 R}$. Most importantly, the pattern of results shows that the invariance violation for controlled processes $C$ for revealed transitions (i.e., whenever substantial explicit knowledge is present) is robust to the change in auxiliary assumptions.

\section{Experiment 3, model $\mathcal{M}_{1 R}$}

For the data of Experiment 3, we additionally fitted model $\mathcal{M}_{1 R}$ analogous to $\mathcal{M}_{1 R}$ of Experiment 2.

Results. The model checks for model $\mathcal{M}_{1 R}$ were satisfactory,

$$
\begin{gathered}
T_{A 1}^{\text {observed }}=689.87, T_{A 1}^{\text {expected }}=657.24, p=.314, \\
T_{B 1}^{\text {observed }}=8.94, T_{B 1}^{\text {expected }}=6.02, p=.263 .
\end{gathered}
$$

and attained a DIC value of $38,881.68$, a value somewhat smaller than the DIC of our extended model $\mathcal{M}_{1}$ presented in the main text and clearly outperforming $\mathcal{M}_{2}$. This again implies that our auxiliary assumptions introduced to $\mathcal{M}_{1 R}$ were much less problematic than the invariance assumption.

Figure C5 shows the parameter estimates obtained from model $\mathcal{M}_{1 R}$. The pattern of results mostly replicates the estimates from model $\mathcal{M}_{1}$; with parameters for controlled processes $C$ being estimated close to zero for nonrevealed transitions.

Figure $\mathrm{C} 6$ shows the posterior differences obtained from model $\mathcal{M}_{1 R}$. The pattern of results again demonstrates robustness of the invariance violation for controlled processes $C$ for revealed transitions (i.e., whenever substantial explicit knowledge was present). There was again some indication of an invariance violation for automatic processes $A$; however, the effect was very small and depended on the specific modeling assumptions.

Appendix D

Specification of priors

This section provides a complete specification of the models and priors used. Code $(\mathbf{R} /$ Stan $)$ is available at https://github.com/methexp/pdl2.

\section{Experiment 1, model $\mathcal{M}_{1}$}

Priors on fixed effects were 

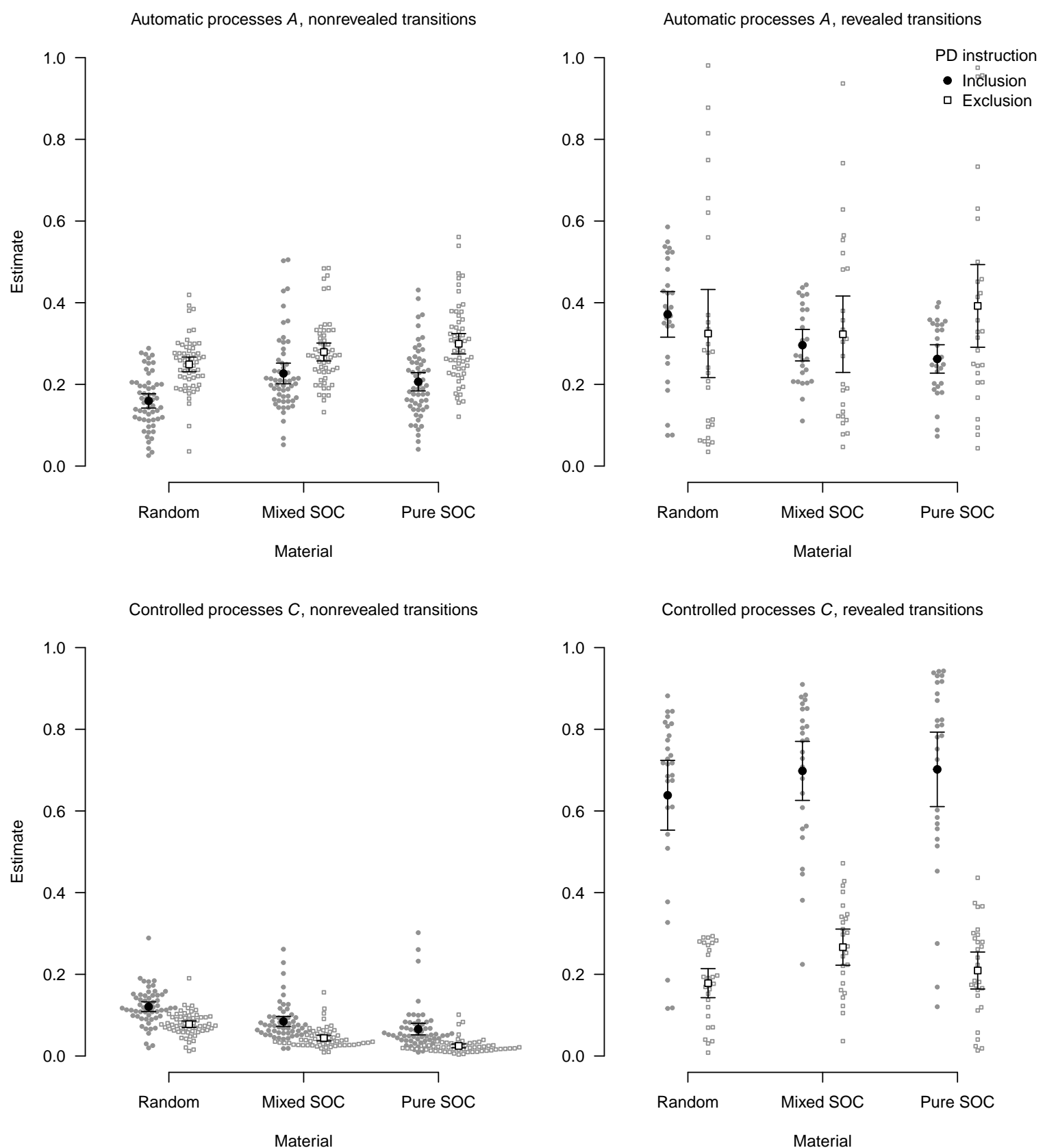

Figure C5. Parameter estimates from Experiment 3, model $\mathcal{M}_{1 R}$. Error bars represent $95 \%$ confidence intervals. 

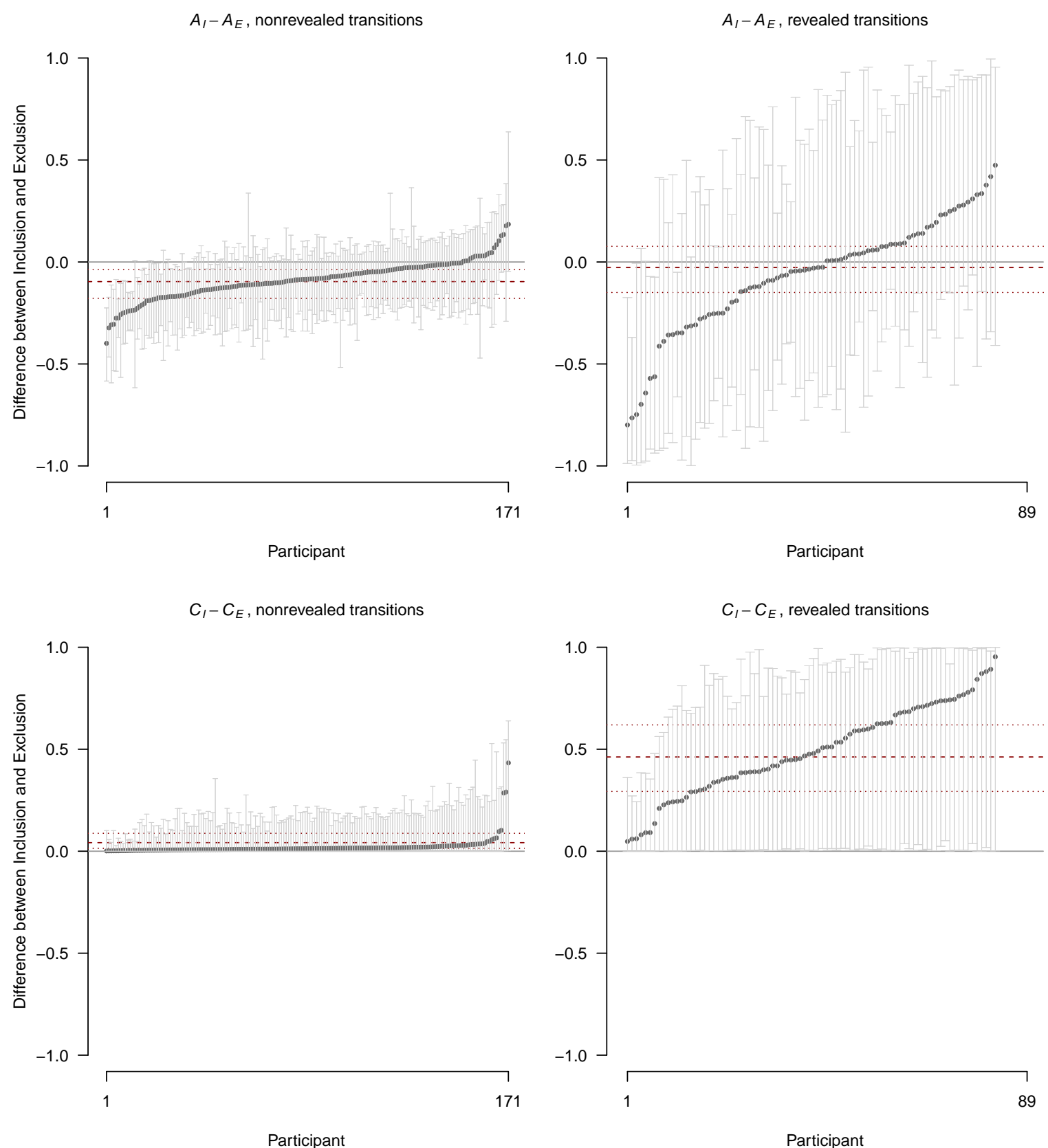

Figure $C 6$. Posterior differences between $A_{I}-A_{E}$ and $C_{I}-C_{E}$ in Experiment 3, model $\mathcal{M}_{1 R}$, plotted for each participant (gray dots) with $95 \%$ credible intervals. Dashed lines represent the posterior means of the differences between mean parameter estimates. Dotted lines represent $95 \%$ credible intervals. 


$$
\begin{aligned}
& \mu_{j l m}^{(C)} \sim N(0,1), j=\{1,2\} ; l=\{1,2\} ; m=\{1,2\} \\
& \mu_{m t}^{(A)} \sim N(0,1), t=\{1, \ldots, 6\} ; m=\{1,2\}
\end{aligned}
$$

where $j$ indexes transition type (revealed \& practiced vs. revealed \& non-practiced), $l$ indexes practice condition (Control, No-practice, Unspecific-practice, Practice, Transfer), $t$ indexes specific items (i.e., transitions), and $m$ indexes $P D$ instruction (inclusion vs. exclusion). Participant effects $\delta_{i m t}^{(A)}$ and $\delta_{i j m}^{(C)}$ can be written as vectors $\boldsymbol{\delta}_{i}$. For participants in the Control group, these were modeled by

$$
\boldsymbol{\delta}_{i} \sim N_{12}\left(0, \Sigma_{l}\right), i=1, \ldots, I
$$

For participants in the No-Practice, Unspecific-Practice, and Practice groups,

$$
\boldsymbol{\delta}_{i} \sim N_{14}\left(0, \Sigma_{l}\right), i=1, \ldots, I
$$

For participants in the Transfer group

$$
\boldsymbol{\delta}_{i} \sim N_{16}\left(0, \Sigma_{l}\right), i=1, \ldots, I
$$

The covariance matrices $\Sigma_{l}$ were modeled separately and independently for each betweensubjects condition. Priors on these matrices were as described below for Experiment 2.

\section{Experiment 2, model $\mathcal{M}_{1}$}

Priors on fixed effects were

$$
\begin{aligned}
& \mu_{k m}^{(C)} \sim N(0,1), k=\{1,2\} ; m=\{1,2\} \\
& \mu_{j k m}^{(A)} \sim N(0,1), j=\{1,2\} ; k=\{1,2\} ; m=\{1,2\}
\end{aligned}
$$

where $j$ indexes transition type (revealed vs. non-revealed), $k$ indexes learning material presented during the SRTT (random vs. probabilistic), and $m$ indexes $P D$ instruction condition (inclusion vs. exclusion). For participants who did not receive explicit knowledge about a single transition, we assumed that all $C_{i j k m}=0$. Therefore, participant effects

are only required for automatic processes $\left(\delta_{i j k m}^{(A)}\right)$. In participants who received explicit knowledge about one transition, two additional participant effects were needed to model controlled processes for revealed transitions $\left(\delta_{i k m}^{(C)}\right)$. We thus provide the specification of participant effects for these two groups of participants separately.

Participants who did not receive explicit knowledge about one transition. For participants who did not receive explicit knowledge about one transition, participant effects $\delta_{i j m}^{(A)}$ can be written as vectors $\boldsymbol{\delta}_{i}$ that were modeled as draws from a multivariate normal

$$
\boldsymbol{\delta}_{i} \sim N_{4}\left(0, \Sigma_{k l}\right), i=1, \ldots, I
$$

where $k$ indexes the learning material that was presented to participant $i$ and $l$ indexes his or her level of the explicit-knowledge factor. The covariance matrices $\Sigma_{k l}$ were obtained from the standard deviations of participant effects $\boldsymbol{\sigma}_{k l}$ and correlation matrices $\Omega_{k l}$ 


$$
\Sigma_{k l}=\operatorname{Diag}\left(\boldsymbol{\sigma}_{k l}\right) \Omega_{k l} \operatorname{Diag}\left(\boldsymbol{\sigma}_{k l}\right), k=\{1,2\}, l=\{1,2\}
$$

Each element $\sigma_{k l p}$ of the vectors of standard deviations $\boldsymbol{\sigma}_{k l}$ was drawn from independent half-normal prior distributions.

$$
\sigma_{k l p} \sim N(0,1)_{\mathcal{I}(0, \infty)}, k=\{1,2\}, l=\{1,2\}
$$

For the correlation matrices $\Omega_{k}$, we used LKJ priors with a scaling factor of 1 (Lewandowski, Kurowicka, \& Joe, 2009):

$$
\Omega_{k l} \sim \operatorname{LKJ} \operatorname{corr}(\nu=1), k=\{1,2\}, l=\{1,2\}
$$

Participants who received explicit knowledge about one transition. For participants who received explicit knowledge about one transition, participant effects $\delta_{i j m}^{(A)}$ and $\delta_{i m}^{(C)}$ can be written as vectors $\boldsymbol{\delta}_{i}$ that were modeled as draws from a multivariate normal

$$
\boldsymbol{\delta}_{i} \sim N_{6}\left(0, \Sigma_{k l}\right), i=1, \ldots, I
$$

where $k$ indexes the learning material that was presented to participant $i$ and $l$ indexes his or her level of the explicit-knowledge factor. The covariance matrices $\Sigma_{k} l$ were specified as above, with the only exception that six instead of four parameters were required.

\section{Experiment 2, model $\mathcal{M}_{2}$}

Priors on fixed effects were

$$
\begin{aligned}
& \mu_{j k l}^{(C)} \sim N(0,1), j=\{1,2\} ; k=\{1,2\} ; l=\{1,2\} \\
& \mu_{j k l}^{(A)} \sim N(0,1), j=\{1,2\} ; k=\{1,2\} ; l=\{1,2\}
\end{aligned}
$$

Participant effects $\delta_{i j}^{(A)}$ and $\delta_{i j}^{(C)}$ can be written as vectors $\boldsymbol{\delta}_{i}$ that were modeled by

$$
\boldsymbol{\delta}_{i} \sim N_{4}\left(0, \Sigma_{k l}\right), i=1, \ldots, I
$$

Priors for the covariance matrix $\Sigma_{k l}$ were specified as above.

Experiment 2, model $\mathcal{M}_{1 R}$

Priors on fixed effects were

$$
\begin{aligned}
& \mu_{j k m}^{(C)} \sim N(0,1), j=\{1,2\} ; k=\{1,2\} ; m=\{1,2\} \\
& \mu_{j k m}^{(A)} \sim N(0,1), j=\{1,2\} ; k=\{1,2\} ; m=\{1,2\}
\end{aligned}
$$

where $j$ indexes transition type (revealed vs. non-revealed), $k$ indexes learning material presented during the SRTT (random vs. probabilistic), and $m$ indexes $P D$ instruction condition (inclusion vs. exclusion). Participant effects $\delta_{i j m}^{(A)}$ and $\delta_{i j m}^{(C)}$ can be written as vectors $\boldsymbol{\delta}_{i}$ that were modeled as draws from a multivariate normal

$$
\boldsymbol{\delta}_{i} \sim N_{8}\left(0, \Sigma_{k l}\right), i=1, \ldots, I
$$


where $k$ indexes the learning material that was presented to participant $i$ and $l$ indexes his or her level of the explicit-knowledge factor. Priors for the covariance matrix $\Sigma_{k l}$ were specified as above.

Experiment 3, models $\mathcal{M}_{1}, \mathcal{M}_{2}$, and $\mathcal{M}_{1 R}$

For the model-based analyses, we used models $\mathcal{M}_{1}, \mathcal{M}_{2}$, and $\mathcal{M}_{1 R}$ analogous to those used in Experiment 2. 